\title{
Multiple-source models for the beams from an Elekta SL25 clinical accelerator
}

\author{
by \\ Zdenko Sego \\ A thesis submitted to the \\ Faculty of Graduate Studies and Research \\ in partial fulfillment of the requirements \\ for the degree of \\ Master of Science \\ Department of Physics \\ Carleton University \\ Ottawa-Carleton Institute of Physics \\ Ottawa, Canada
}

April 26, 2006

Copyright (C) 2006 Zdenko Sego 


$\begin{array}{ll}\begin{array}{l}\text { Library and } \\ \text { Archives Canada }\end{array} & \begin{array}{l}\text { Bibliothèque et } \\ \text { Archives Canada }\end{array} \\ \begin{array}{l}\text { Published Heritage } \\ \text { Branch }\end{array} & \begin{array}{l}\text { Direction du } \\ \text { Patrimoine de l'édition }\end{array} \\ \begin{array}{l}\text { 395 Wellington Street } \\ \text { Ottawa ON K1A ON4 }\end{array} & \begin{array}{l}\text { 395, rue Wellington } \\ \text { Ottawa ON K1A ON4 } \\ \text { Canada }\end{array}\end{array}$

Your file Votre référence ISBN: 978-0-494-18376-2 Our file Notre référence ISBN: 978-0-494-18376-2

NOTICE:

The author has granted a nonexclusive license allowing Library and Archives Canada to reproduce, publish, archive, preserve, conserve, communicate to the public by telecommunication or on the Internet, loan, distribute and sell theses worldwide, for commercial or noncommercial purposes, in microform, paper, electronic and/or any other formats.

The author retains copyright ownership and moral rights in this thesis. Neither the thesis nor substantial extracts from it may be printed or otherwise reproduced without the author's permission.
AVIS:

L'auteur a accordé une licence non exclusive permettant à la Bibliothèque et Archives Canada de reproduire, publier, archiver, sauvegarder, conserver, transmettre au public par télécommunication ou par l'Internet, prêter, distribuer et vendre des thèses partout dans le monde, à des fins commerciales ou autres, sur support microforme, papier, électronique et/ou autres formats.

L'auteur conserve la propriété du droit d'auteur et des droits moraux qui protège cette thèse. $\mathrm{Ni}$ la thèse ni des extraits substantiels de celle-ci ne doivent être imprimés ou autrement reproduits sans son autorisation.
In compliance with the Canadian

Privacy Act some supporting forms may have been removed from this thesis.

While these forms may be included in the document page count, their removal does not represent any loss of content from the thesis.
Conformément à la loi canadienne sur la protection de la vie privée, quelques formulaires secondaires ont été enlevés de cette thèse.

Bien que ces formulaires aient inclus dans la pagination, il n'y aura aucun contenu manquant.

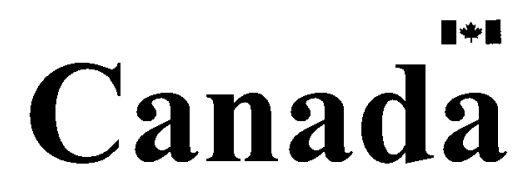




\section{Abstract}

The accurate calculations of the absorbed dose distribution in patients irradiated by clinical beams is very important in radiotherapy treatment. Currently, the most accurate method of radiation therapy dose calculation is the Monte Carlo (MC) simulation of radiation transport, which requires detailed information on the character of the beam in the form of a phase-space file, which imposes very high requirements for disk space and CPU time. Monte Carlo calculations of dose distribution using multiple-source models is an alternative to using the phase-space information as direct input to the dose calculation code.

Several multiple-source models for electron and photon beams of an Elekta Precise SL25 linear accelerator, located at the National Research Council of Canada (NRC), have been developed by simulating radiation transport through the accelerator. Calculation results were presented, and the difference between central-axis depth-dose and dose-profile values calculated from the phase-space file and ones from the experiment were compared.

The corresponding phase-space data enabled the characterization of several sources representing the main components of the beam defining system. These simplified beam models have been used to generate Monte Carlo dose distributions, and, to compare to those calculated with full phase space data for photon and electron beams. It was shown that agreement in dose calculations between the models and original phase-space file is well within the statistics of $1.0 \%$ for all sub-source models.

The BEAMDP code and associated MORTRAN replacement macros to create multiple-source models has been modified. Modifications include the separation of spatial binning inside and outside the treatment field to allow better resolution inside 
and around the edge of the treatment field and multiple-energy spectra to handle the case when the mean energy varies significantly in different spatial regions of the beam. The difference in dose calculations were presented between the two versions of the code. The new BEAMDP code proved to give much better results in the situation where the mean energy varies with the distance of axis as in the case with photon beams from the Elekta SL25 linear accelerator. 


\section{Acknowledgments}

I would like to thank to my supervisor, David W.O. Rogers, for his teaching, invaluable guidance and helpful suggestions throughout this project, for giving me the possibility to take part in activities of Carleton Laboratory for Radiotherapy Physics group.

I would also like to thank to Dr. Malcolm McEwen at the Ionizing Radiation Standards group (IRS) at the National Research Council of Canada for experimental data for Elekta SL25 linear accelerator, Mr. Blake Walters for initial electron input files and Dr. Iwan Kawrakow for photon input files.

Thanks to all the wonderful people at Carleton University in Ottawa for the friendly and stimulating environment they provided for my work. 


\section{Contents}

1 Introduction 1

1.1 Monte Carlo in radiotherapy . . . . . . . . . . . . . . . 1

1.2 The EGSnrc Monte Carlo system . . . . . . . . . . . . . 5

1.2.1 Transport mechanism in the EGSnrc code system . . . . . . 6

1.2.2 Photon transport . . . . . . . . . . . . . 7

1.2.3 Electron transport . . . . . . . . . . . . . 9 9

1.2.4 Random number generators ............... 10

1.2.5 Material data sets and the PEGS4 code . . . . . . . . . 10

1.2.6 Energy cutoffs . . . . . . . . . . . . . . . . . 11

1.2.7 Variance reduction techniques .............. 12

1.2.8 Parallel computing . . . . . . . . . . . . . . . . . 12

1.3 BEAMnrc code . . . . . . . . . . . . . . . . . . 13

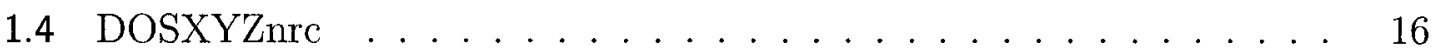

1.5 BEAMDP code ....................... . 17

vi 
1.6 Thesis Overview . . . . . . . . . . . . . . . . . . 18

2 Elekta SL25 linear accelerator $\quad 20$

2.1 General introduction ................... 20

2.2 Monte Carlo modeling of Elekta SL25 linac . . . . . . . . . . . . . 23

2.2.1 Geometry details and schematic diagram . . . . . . . . . 26

2.3 Water phantom measurements . . . . . . . . . . . 30

2.4 Elekta SL25 photon beams . . . . . . . . . . . . . . . . 32

2.5 Elekta SL25 electron beams . . . . . . . . . . . . . . . 35

3 Multiple-source model $\quad 41$

3.1 About multiple-source models . . . . . . . . . . . . . . . . . . . 42

3.1.1 Particle Origin . . . . . . . . . . . . . . 43

3.1.2 Method of beam model design .............. 44

3.2 Types of source models . . . . . . . . . . . . . . . . . 45

3.2.1 Ring or point sub-sources _. . . . . . . . . . . . 46

3.2.2 Applicator sub-sources . . . . . . . . . . . . . . . 47

3.2.3 Collimator sub-sources . . . . . . . . . . . . . 47

3.2 .4 Planar sub-sources . . . . . . . . . . . . . . . 48

3.3 Planar fluence . . . . . . . . . . . . . . . . . 48

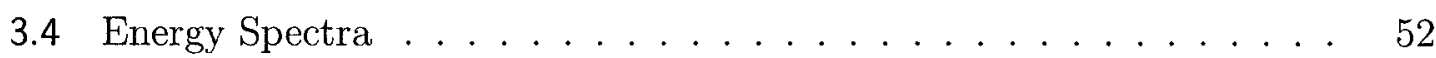

vii 
3.5 Pin-hole method .................... 57

3.6 Beam reconstruction . . . . . . . . . . . . . . 58

3.7 Phase-space reconstruction . . . . . . . . . . . . . 60

3.8 Model verification . . . . . . . . . . . . . . . . 61

4 Monte Carlo calculations $\quad 62$

4.1 BEAMnrc calculations ..................... 63

4.1 .1 Photon beams . . . . . . . . . . . . . 65

4.1 .2 Electron beams .................. 70

4.2 BEAMDP calculations .................... 71

4.3 DOSXYZnrc calculations ...................... 73

4.4 Phase-space reconstruction . . . . . . . . . . . . . 74

4.5 Calculation results .................... 77

$\begin{array}{lll}5 & \text { Original phase space file analysis } & 78\end{array}$

5.1 Electron beams ........................ 78

5.2 Photon beams ........................ 85

5.3 Conclusion . . . . . . . . . . . . . . . . 88

6 Models and results $\quad 89$

6.1 Electron beam . . . . . . . . . . . . . . . . 92

6.1 .1 Models ...................... 92

viii 
6.1.2 Depth-dose and dose-profile comparisons . . . . . . . . . . 96

6.2 Photon beam ........................ 101

6.2 .1 Models ....................... 101

6.2.2 Depth-dose and dose-profile comparisons . . . . . . . . . 106

7 Conclusion 


\section{List of Tables}

2.1 BEAMnrc photon and electron beams simulation for the NRC Elekta SL25 treatment head . . . . . . . . . . . . . . 23

2.2 Components in the treatment head . . . . . . . . . . . . . . 24

2.3 Component Modules (CM) in the treatment head . . . . . . . . 29

2.4 Details of the incident electron energies . . . . . . . . . . . 36

4.1 Number of histories in photon beam simulations . . . . . . . . . . 66

4.2 Number of histories in electron beam simulations . . . . . . . . 70

4.3 Geometry used to calculate dose distributions with DOSXYZnrc for photon beams ........................... 75

4.4 Geometry used to calculate dose distributions with DOSXYZnrc for

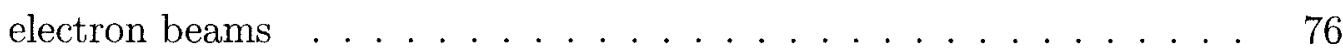

6.1 Parameters for the Elekta SL25 multiple-source models . . . . . . . . 91

6.2 Components of the full multiple-source model for the $18 \mathrm{MeV}$ electron

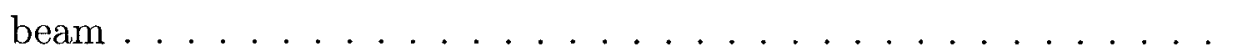


6.3 Components of the full electron-photon multiple-source model for 18 $\mathrm{MeV}$ electron beam . . . . . . . . . . . . . . . . . . . 94

6.4 Components of the reduced multiple-source model for $18 \mathrm{MeV}$ electron

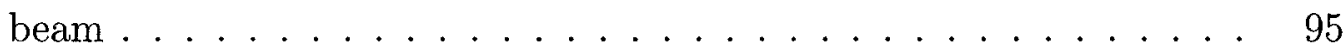

6.5 Components of the optimized multiple-source model for $18 \mathrm{MeV}$ elec-

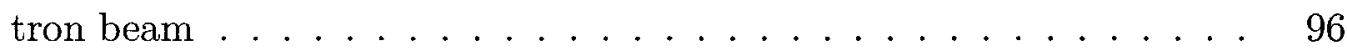

6.6 Components of the two-source model for $18 \mathrm{MeV}$ electron beam . . . 96

6.7 Components of the full multiple-source model for $25 \mathrm{MV}$ photon beam 102

6.8 Components of the reduced full multiple-source model for $25 \mathrm{MV}$ pho-

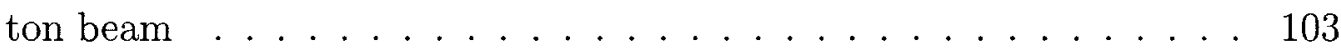

6.9 Components of the further reduced multiple-source model for $25 \mathrm{MV}$ photon beam . . . . . . . . . . . . . . 104

6.10 Components of the optimized multiple-source model for $25 \mathrm{MV}$ photon

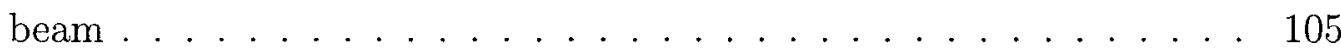

6.11 Components of the three-source model for $25 \mathrm{MV}$ photon beam $\ldots 105$ 


\section{List of Figures}

1.1 Calculation of dose distribution in a patient .......... 2

1.2 EGS_windows presentation of simulated Elekta SL25 . . . . . . . . 15

2.1 Schematic diagram for Elekta SL25 . . . . . . . . . . . . 27

2.2 Phantom setup for a dosimetry measurements . . . . . . . . . . 30

2.3 6 MV photon beam, $10 \times 10 \mathrm{~cm}^{2}$ comparison: BEAMnre versus experiment 33

2.4 10 MV photon beam, 10x10 $\mathrm{cm}^{2}$ comparison: BEAMnrc versus experiment ............................. 34

2.5 25 MV photon beam, 40x40 $\mathrm{cm}^{2}$ comparison: BEAMnrc versus experiment ............................. 34

2.6 Effect of incident electron energy distribution on depth-dose curve . . 37

2.7 Electron $4 \mathrm{MeV}, 10 \times 10 \mathrm{~cm}^{2}$ comparison: BEAMnrc versus experiment 37

2.8 Electron $8 \mathrm{MeV}, 10 \times 10 \mathrm{~cm}^{2}$ comparison: BEAMnrc versus experiment 38

2.9 Electron $12 \mathrm{MeV}, 10 \times 10 \mathrm{~cm}^{2}$ comparison: BEAMnrc versus experiment 38

2.10 Electron $18 \mathrm{MeV}, 10 \times 10 \mathrm{~cm}^{2}$ comparison: BEAMnrc versus experiment 39 
2.11 Electron $22 \mathrm{MeV}, 10 \times 10 \mathrm{~cm}^{2}$ comparison: BEAMnrc versus experiment 39

3.1 Beam model design flowchart . . . . . . . . . . . . . . . . 44

3.2 Field types for planar fluence scoring . . . . . . . . . . . . . . 49

3.3 Comparison for the planar fluence scoring techniques . . . . . . . . 51

3.4 Comparison for the mean energy distribution . . . . . . . . . . . 54

3.5 Comparison of the electron energy spectra . . . . . . . . . 55

3.6 Comparison of the photon energy spectra . . . . . . . . . . 56

3.7 Pinhole method . . . . . . . . . . . . . 57

4.1 6 MV photon beam: BEAMnrc versus experiment . . . . . . . . 67

$4.210 \mathrm{MV}$ photon beam: BEAMnrc versus experiment . . . . . . . 67

4.3 25 MV photon beam: BEAMnrc versus experiment . . . . . . . 69

4.4 The geometry used for the DOSXYZnrc calculations . . . . . . . 73

5.1 Electron spectral distribution . . . . . . . . . . . 79

5.2 Electron planar fluence . . . . . . . . . . . . . . . . 81

5.3 Photon spectral distribution and planar fluence . . . . . . . . . 83

5.4 Photon spectral distribution and planar fluence . . . . . . . . 84

5.5 Photon and electron planar fluence from 25 MV photon beam, $40 \mathrm{x} 40 \mathrm{~cm}^{2}$ field size ..................... 85

5.6 Electron spectral distribution from $25 \mathrm{MV}$ photon beam . . . . . . 87 
5.7 Mean energies of photons and electrons from $25 \mathrm{MV}$ photon beam . .

6.1 Central-axis depth-dose comparison: phase-space file versus models . $\quad 97$

6.2 Dose-profile comparison: phase-space file versus models . . . . . . . . 98

6.3 Central-axis depth-dose comparison: phase-space file versus one-source

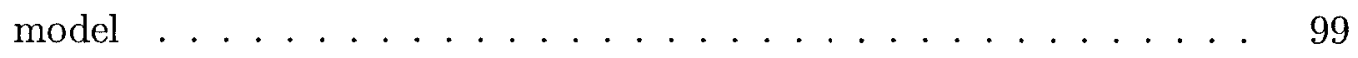

6.4 Central-axis depth-dose comparison: new versus old BEAMDP code . 100

6.5 Dose-profile comparison for the electron beam: new versus old BEAMDP code ......................... 100

6.6 Central-axis depth-dose comparison: phase-space file versus models . 106

6.7 Dose-profile comparison: phase-space file versus models . . . . . . . . 107

6.8 Central-axis depth-dose comparison: phase-space file versus optimized

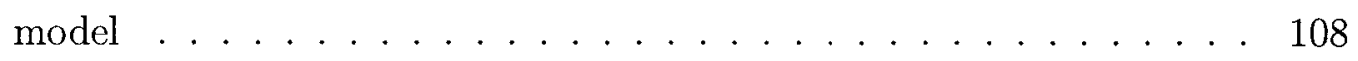

6.9 Absorbed dose to water for $25 \mathrm{MV}$ broad beam photon beam . . . . . 108

6.10 Central-axis depth-dose comparison: phase-space file versus one-source

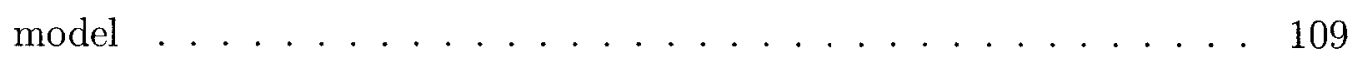

6.11 Dose-profile comparison for the photon beam: new versus old BEAMDP code . . . . . . . . . . . . . . . 110

7.1 Disk space requirement, phase-space versus multiple-source model . . 112 


\section{Chapter 1}

\section{Introduction}

\subsection{Monte Carlo in radiotherapy}

Clinically, the most important quantity to calculate in radiotherapy is the dose distribution per monitor unit in patients undergoing cancer treatment by ionizing radiation. Optimization of the therapeutic gain of radiation, i.e. maximizing the dose to tumors, while at the same time minimizing dose to healthy tissues, depends critically on the accuracy of the dose calculation. It has been shown that an under-dosage of $5 \%$ in the dose delivered to the tumour can decrease the tumour probability control by more than $15 \%$ while an increase of $5 \%$ in the dose delivered to the tumour can cause intolerable side effects in the surrounding healthy tissues.

Although the physics of radiation transport is very well known, analytical methods for dose calculations are associated with large errors, especially in heterogeneous patient tissues where the effect of electron transport cannot be handled sufficiently accurately. 
The most accurate method of radiation therapy dose calculation is based on Monte Carlo simulation of radiation transport. The Monte Carlo method is a numerical technique based on random number sampling to simulate a stochastic process, in this case, transport of photon and electron particles through a medium. The major advantage of this method is that one can score many quantities of interest, even more than are physically measurable. For example, one can score not only energy deposition in a water tank, but also the number of particles scattered in a particular region before depositing energy in a small volume of the water phantom. To achieve the full

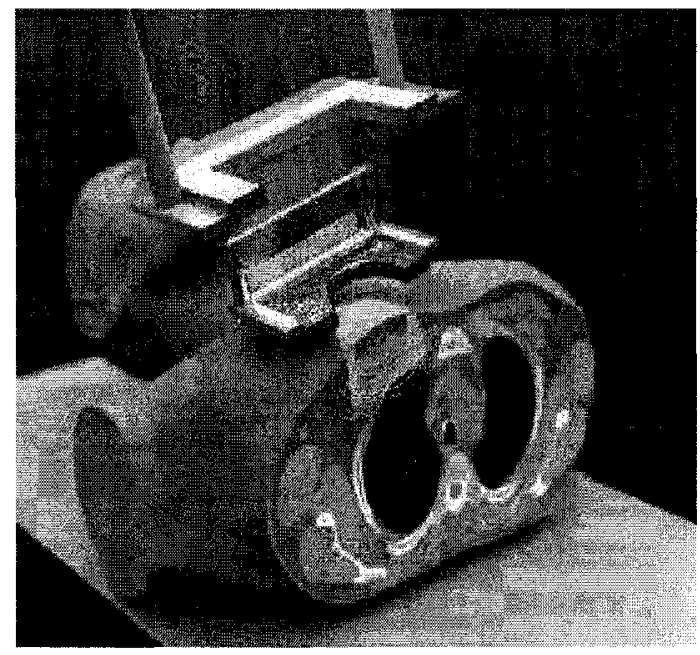

Figure 1.1: Cutaway rendering of an electron beam dose calculation for the chest wall. The goal is to adjust beam parameters to avoid dose to the lung. Rendering produced by Thomas Lundberg of $M D S$-Nordion

accuracy of a Monte Carlo dose calculation, detailed information on the character of the beam is required, which includes the energy, angular, and spatial distributions of all particles in the clinical beam. This is phase-space information which is stored in a phase-space file. The phase-space storage requirement for one particle is 28 bytes.

\subsection{MONTE CARLO IN RADIOTHERAPY}


Considering that a million electrons must be followed in a Monte Carlo dose calculation to get $1 \%$ statistical accuracy $(1 \sigma)$ in the central-axis dose of a $10 \times 10 \mathrm{~cm}^{2}$ field in a $1 \mathrm{~cm}^{3}$ voxel size, then the requirements for storing a phase-space file is $28 \mathrm{MB}$. If bremsstrahlung photons are also included then the phase-space information grows to over $100 \mathrm{MB}$ for an electron beam and several GB for a photon beam (Tables 4.1 and 4.2)

This requires at least $40 \mathrm{~GB}$ disk space to store the 56 beams from an accelerator with 5 electron beam energies and 3 photon beam energies with or without 3 different sizes of applicators and one broad beam (no applicators, jaws wide open) for each energy. Additionally, for dose calculations which use smaller voxels size, the phase-space storage will be significantly increased to achieve the same level of accuracy. Besides the high storage requirements, a single Monte Carlo simulation can take several days to complete. Simulation of all 56 beams mentioned above would represent a considerable burden on computing resources.

Beam modeling represents an alternative to using the phase-space information as direct input to the dose calculation code. A multiple-source model is a beam model based on the fact that particles from the same component of a linear accelerator have very similar characteristics, in terms of energies and incident directions, and that particles from different components have different energy, angular and spatial distributions. ${ }^{1,2}$

Beam modeling can save considerably on both disk space and computing time. The size of the multiple-source model file is negligible compared to the size of phasespace file. On the other hand, beam models can significantly shorten the computation time in the treatment head simulation to achieve the same level of precision.

\subsection{MONTE CARLO IN RADIOTHERAPY}


There are several published studies which are based on multiple-source models that appear to be attractive because of significant reduction in calculation time and data storage. For example: hybrid commissioning approach based on multiple-source model for Monte Carlo treatment planning ${ }^{3}$ as a time saving method for commissioning of the same type of accelerators based on models developed from only one simulated phase-space, and application in narrow beam radio-surgery ${ }^{4}$ where full Monte Carlo simulation of the beam is of very poor efficiency with a great limitation in time and storage capacity; multiple-source models enhance the quality of the reconstructed beam, and at the same time reduce the time and storage capacity.

The traditional major drawbacks of the Monte Carlo method for calculating dose distribution in a phantom are long computation times and the need for a detailed knowledge of the incident radiation beam. Many improvements in computer technology and in Monte Carlo codes keep reducing these limitations. Monte Carlo is becoming a part of today's state-of-the-art photon and electron treatment planning systems by leading vendors in this field. It is likely that several years from now all commercial treatment planning systems will be based on Monte Carlo methods of dose calculation. ${ }^{5}$ If beam models introduce only a little error in the dose calculation then they will find a wider use in the modern Monte Carlo systems as well.

The purpose of the present work is to develop an accurate multiple-source model for an Elekta SL25 medical linear accelerator located at the National Research Council of Canada (NRC) that will be useful for research on the NRC machine. Models are validated against the phase-space file, which in turn should be validated against measurements. The source model is developed based on a detailed modeling of the accelerator using the well-established EGSnrc ${ }^{6}$ based BEAMnrc code. ${ }^{7}$

The BEAMDP (BEAM Data Processor) code is used to develop the source

\subsection{MONTE CARLO IN RADIOTHERAPY}


model. This code utilizes the BEAMnrc-based phase space information of the accelerator scored at the patient plane. The BEAMDP program distributed along with the BEAMnrc system utilizes a simple algorithm to develop source models. In the present work, many routines in the BEAMDP that are related to sampling of particles inside and outside the treatment field were modified. This is needed to obtain a better model for the accelerator, the details of which are described in Chapter 3.

\subsection{The EGSnrc Monte Carlo system}

Generally, a Monte Carlo simulation of radiation transport has four major components:

1. Cross-section data for simulating physical processes

2. The algorithms for the particle transport

3. Geometry specification

4. Statistical analysis

The physics of the Monte Carlo simulation is contained in the two first components. Cross-section data contains information regarding the interaction properties of the particle and the media through which it travels. A particle history is started by creating the particle with position and energy coordinates according to a specified source distribution. The particle travels a certain distance before undergoing an interaction. The type of interaction and the resulting particles are determined by the interaction cross-sections at that point. Any secondary particles created must also be transported and a particle history ends when all particles have either deposited their

\subsection{THE EGSNRC MONTE CARLO SYSTEM}


energy within the medium or have left the geometry. By simulating a large number of histories Monte Carlo calculation can accurately predict the average values of macroscopic quantities of interest such as energy deposition. The last two components do not affect the underlying physics but may affect calculation time of the simulation.

There are many Monte Carlo systems for simulating radiation transport. EGSnrc, ${ }^{8-10}$ EGS4 $^{9}{ }^{9}$ ETRAN/ITS, ${ }^{11-13}$ MCNP $^{14}$ and PENELOPE ${ }^{15,16}$ are general purpose Monte Carlo codes. Other codes are primarily designed for use in medical applications for dose calculation, such as X-ray Voxel Monte Carlo (XVMC) ${ }^{17,18}$ and Dose Planning Method (DPM). ${ }^{19}$

The EGSnrc code system is widely applied in radiotherapy dose calculation. It is an integral part of many current studies in radiation dosimetry, radiotherapy physics and radiation protection, shown to be accurate to within $0.1 \%$ with respect to its own cross-sections. ${ }^{20,21}$

\subsubsection{Transport mechanism in the EGSnrc code system}

The EGSnrc code system (Electron-Gamma Shower) ${ }^{10,22}$ is a general purpose Monte Carlo code system used for the simulation of the coupled transport of electrons and photons through an arbitrary geometry. It is an improved version of its predecessor, the EGS4 ${ }^{9}$ system with significant advances in several aspects of electron transport: new electron transport algorithm PRESTA-II, improved multiple-scattering theory which includes relativistic spin effects in the cross section, electron impact ionization, more accurate boundary crossing algorithm, and improved sampling algorithms for a variety of energy and angular distributions. The detailed description of these features of the EGSnrc system can be found in the EGSnrc manual ${ }^{10}$ and the manual for the

\subsection{THE EGSNRC MONTE CARLO SYSTEM}


multi-platform version of the EGSnrc system. ${ }^{23}$

Monte Carlo simulation of electron and photon transport must take into account all relevant physical processes for both particles.

\subsubsection{Photon transport}

As a photon passes through matter it can interact in a variety of processes. The four major processes are:

- photoelectric absorption

- coherent or Rayleigh scattering

- incoherent or Compton scattering

- pair production

At lower photon energies the dominant photon interaction is photoelectric absorption. In this interaction the photon is absorbed by the atom and a photoelectron is emitted. The energy of the emitted electron is equal to the energy of incident photon lowered by its binding energy. The atom is left in an excited state with a vacancy in the ionized shell, usually in the $\mathrm{K}$ shell. The atom may relax by emitting fluorescent photons or by emitting Auger or Coster-Kronig electrons. EGSnrc uses the total photo-absorption cross-section taken from the compilation by Storm and Israel $^{24}$ with fluorescent relaxation in the $\mathrm{K}$ shell. The user has an option to select detailed simulation of relaxation in the $K, L, M$ shells

In coherent or Rayleigh scattering the photon scatters elastically from atoms, molecules or other structures of the medium. No energy is lost by the photon, ex-

\subsection{THE EGSNRC MONTE CARLO SYSTEM}


cept a negligible amount to atomic recoil. This scattering occurs at low energies. EGSnrc treats Rayleigh scattering using the total coherent scattering cross-sections from Storm and Israel.

Incoherent or Compton scattering occurs at almost all energies. It dominates near $1 \mathrm{MeV}$. A photon scatters from an atomic electron and sets it in motion. In many calculations electrons may be considered as free electrons. At lower energies the cross sections are effected by the fact that the electron is actually bound in an atom. The treatment of Compton scattering in EGSnrc is based on the relativistic impulse approximation which includes binding effect, or optionally, modeling electrons as free, according to the Klein-Nishina formula.

Pair production in the electro-magnetic field of the nuclei and atomic electrons occurs at energies above $1.022 \mathrm{MeV}$. If a photon interacts with the field of a nucleus, the photon is absorbed and creates an electron-positron pair. Much less frequently the photon may interact with the field of an atomic electron. In this case, the electron can take a considerable amount of energy and leave the atom with a vacancy. This process is also known as triplet production. EGSnrc uses the extreme relativistic Born approximation differential cross-sections. ${ }^{25}$ Triplet production is not simulated explicitly, but approximated by sampling the distance to subsequent pair production using pair plus triplet cross-sections.

There are other photon processes that can occur, but which are not of significant importance in radiotherapy: double Compton effect, in which a second photon appears when a photon scatters from an electron, nuclear Thomson scattering, in which a photon scatters elastically from the nucleus, photo-nuclear interaction in which the nucleus absorbs a photon and gives off a nucleon and Delbruck scattering in which a photon scatters elastically from the Coulomb field of the nucleus.

\subsection{THE EGSNRC MONTE CARLO SYSTEM}




\subsubsection{Electron transport}

Electrons lose energy in matter by two basic processes, inelastic collisions with atomic electrons and by bremsstrahlung emission in the field of the atomic nuclei. Inelastic collisions are more dominant at low energies, while radiative energy loss is more important at higher energies.

Transport of electrons presents some difficulties since an electron may undergo hundreds of thousands of interactions as it slows down in a medium. For example, electrons undergo about 4000 elastic scatterings in slowing down from 500 to $250 \mathrm{keV}$ in aluminum, and about 7000 in gold. ${ }^{26}$ The computation time required for event-byevent transport of this slowing down process would be impractical. This problem is dealt with in EGSnrc by using the condensed history technique described by Berger. ${ }^{11}$ In this method, a large number of individual interactions are condensed into a single step. The overall effect of this step is sampled from the relevant multiple scattering distributions. The condensed history technique is a reasonable approximation, since in most cases a single interaction causes little change in the energy and direction of an electron. More details can be found in the work of Kawrakow and Bielajew. ${ }^{27}$ EGSnrc uses the PRESTA-II condensed history scheme for the simulation of electron transport. Bremsstrahlung processes that result in the creation of photons above a photon energy threshold $A P$ and inelastic collisions that set in motion atomic electrons with kinetic energy above an electron energy threshold $A E$ are both simulated explicitly and the secondary particles are transported.

\subsection{THE EGSNRC MONTE CARLO SYSTEM}




\subsubsection{Random number generators}

The "pseudo" random number generator (RNG) is the heartbeat of Monte Carlo simulation. It is what generates the pseudo-random nature of Monte Carlo simulation thereby imitating the true stochastic nature of particle interactions. Random numbers are generated every time an interaction distribution is sampled.

EGSnrc is supplied with two random number generators: RANLUX and RANMAR. The generator used with EGS4 is RANMAR, while the default generator for EGSnrc is RANLUX which comes with the variety of "luxury levels" for the random number sequences. The details of each of these generators are described elsewhere. ${ }^{28-31}$ The important features of these random number generators are that they are completely portable, producing the same random number sequence on different machines and that they can be initialized to guarantee independent random number sequences when doing parallel computing.

\subsubsection{Material data sets and the PEGS4 code}

The cross section data for EGSnrc is created by the code PEGS4 (Preprocessor for EGS). PEGS4 is a stand-alone utility program that is used to generate material data files containing the cross-section information for the materials of interest in the calculations. ${ }^{9}$ When a material data set is generated using PEGS4, lower energy bounds for the production of secondary electrons and photons, $A E$ and $A P$ respectively, are defined. These parameters represent the lowest energy for which the material data are generated. The corresponding upper bounds are $U E$ and $U P$.

\subsection{THE EGSNRC MONTE CARLO SYSTEM}




\subsubsection{Energy cutoffs}

There are a variety of transport parameters in EGSnrc that must be specified for each simulation. Among these are the electron and photon cutoff energies, ECUT and PCUT respectively. A cutoff energy is the energy at which the Monte Carlo simulation stops transport of the particle if the particle's energy falls below the cutoff value.

ECUT defines the global cutoff energy for electron transport in $\mathrm{MeV}$. As soon as an electron's total energy falls below the cutoff energy, its history is terminated and its energy deposited in the current region. The time required for a given calculation is strongly dependent on the value of ECUT and thus it is important to use as high a value as possible. The electron cutoff energy is equal to

$$
E C U T=E_{k}+m c^{2}
$$

where $E_{k}$ is the kinetic energy of electron and $m c^{2}$ the electron mass equivalent. $E C U T$ defaults to $A E$ for a given medium - the lowest electron energy for the data in the PEGS4 data.

PCUT defines the global cutoff energy for photon transport in $\mathrm{MeV}$. It is the photon equivalent of ECUT. PCUT defaults to $A P$ for the given medium - the lowest photon energy for which there are data in the PEGS4 file.

Transport is also controlled by parameters $A E$ and $A P$ in the PEGS4 material data since they determine the cutoff energy for electrons and photons respectively below which secondary particles are not created and below which transport will stop, irrespective of the values of ECUT and PCUT.

\subsection{THE EGSNRC MONTE CARLO SYSTEM}




\subsubsection{Variance reduction techniques}

A Monte Carlo simulation can be prohibitively time-consuming when a large number of particle histories is required in order to achieve the desired level of precision. The efficiency of Monte Carlo calculation is given by the equation

$$
\epsilon=\frac{1}{T \sigma^{2}}
$$

where $\sigma^{2}$ is the statistical uncertainty of the quantity of interest and $T$ is the CPU time to complete the calculation.

Techniques that improve the efficiency $\epsilon$ of a calculation without altering the physics are known as variance reduction techniques. Monte Carlo codes also use other techniques to shorten the computation time, such as range rejection and high cutoff energies. The basic method for range rejection is to calculate the range of a charged particle and terminate its history (depositing all of its energy at that point) if it can not leave the current region. These techniques only approximate the transport and are biased. The variance reduction techniques available with the EGSnrc system are described in detail in the EGSnrc users manual. ${ }^{10}$

Variance reduction techniques of particular interest to the present work are described in Chapter 4, in particular, the directional bremsstrahlung splitting (DBS) variance reduction technique used in simulation of clinical photon beams from an Elekta SL25 linear accelerator.

\subsubsection{Parallel computing}

Monte Carlo calculations with the EGSnrc system are easily run in parallel. Single simulations can be executed simultaneously on multiple computers in order to reduce

\subsection{THE EGSNRC MONTE CARLO SYSTEM}


the elapsed time to complete the simulation, ensuring that random number generators on each computer are initialized to guarantee independent random number sequences.

If a job is submitted for parallel-processing, each computer is initially assigned a small fraction of the total number of histories. The parallel-processing subroutine controls the simulation on each computer, observing how many histories have been completed and how many remain. As each CPU finishes an assigned number of histories, it will be given the next fraction of histories to execute until the required total number has been reached. When all histories have been completed, the individual output files from the different CPUs are combined to give a final output file.

The advantage of this approach is that it makes efficient use of computers having different computational power. Some calculations performed in this study are performed using parallel processing on ten computers. A typical simulation for photon beams that would be running several days on one computer, takes 20-30 hours on ten computers.

\subsection{BEAMnrc code}

BEAMnrc, ${ }^{32}$ which is an improved version of the BEAM code, ${ }^{7}$ is a special-purpose Monte Carlo simulation code which is based on the EGSnrc Monte Carlo system. BEAMnrc allows the simulation of radiotherapy external beam treatment units and produces data on realistic clinical beams. The BEAMnrc code has been designed to simulate radiation beams from any radiotherapy source, including low-energy $\mathrm{x}$-rays, ${ }^{60} \mathrm{Co}$ units, and both photon and electron beams from a wide variety of accelerators. It has been extensively benchmarked against measured dose distributions for a variety of accelerators and good agreement was obtained for central-axis percentage depth-dose

\subsection{BEAMNRC CODE}


as well as in dose-profile curves. ${ }^{7}$

The BEAMnrc code is written in MORTRAN3, a FORTRAN77 preprocessor which is used for the EGS4/EGSnrc systems. The BEAMnrc system runs under UNIX, Linux or MS Windows operating systems.

A basic element of the BEAMnrc design is that the model is built up from a series of component modules (CMs), each of which operates completely independently of the others. Each CM deals with a specific class of geometric shapes but does not specify explicit values related to the geometry. Once the model is built it can be used to simulate a wide variety of configurations since each simulation reads a user specified input file which can be created using a graphical user interface. Component modules (CM) that were used in BEAMnrc simulation modeling an Elekta SL25 linear accelerator are described in Chapter 2.

BEAMnrc has three major forms of output: listing output file, phase-space file and graphics file. The listing output file is always created during a simulation. It contains all information input for an accelerator simulation, an accurate specification of exactly what accelerator model was used and geometric parameters for each of the component modules. The phase-space file output is optional. It contains data about each individual particle crossing the scoring plane, i.e., the position, energy, direction, charge, weight and history tag:

$$
\left\{H_{i} \mid i=1,2,3, . . N\right\} \text { with } H_{i}=\left(x_{i}, y_{i}, u_{i}, v_{i}, q_{i}, E_{i}, \text { weight }_{i}, \mathrm{LATCH}_{i}\right)
$$

This phase space file can be re-used by BEAMnrc or analyzed using a data analysis program such as BEAMDP ${ }^{1}$ or PAW. ${ }^{33}$ To allow flexible access to the particle's history, BEAMnrc includes a general technique built on the LATCH feature of EGSnrc. A LATCH variable is associated with each particle, it is passed to a particle's de- 
scendents and can be interrogated or modified by the user. In BEAMnrc, each bit of LATCH is manipulated separately. The LATCH technique allows one to separate effects of particles which have entered a water phantom from those that have been produced from particle interactions in the phantom, etc. An optional BEAMnrc output is a graphics file with a simple representation of the geometry for input to the EGS_Windows graphics package. The user can request the output of a complete history file which tracks every step in the simulation along with an output data file which can be read by the EGS_Windows application and displayed in three dimensional space (Figure 1.2).

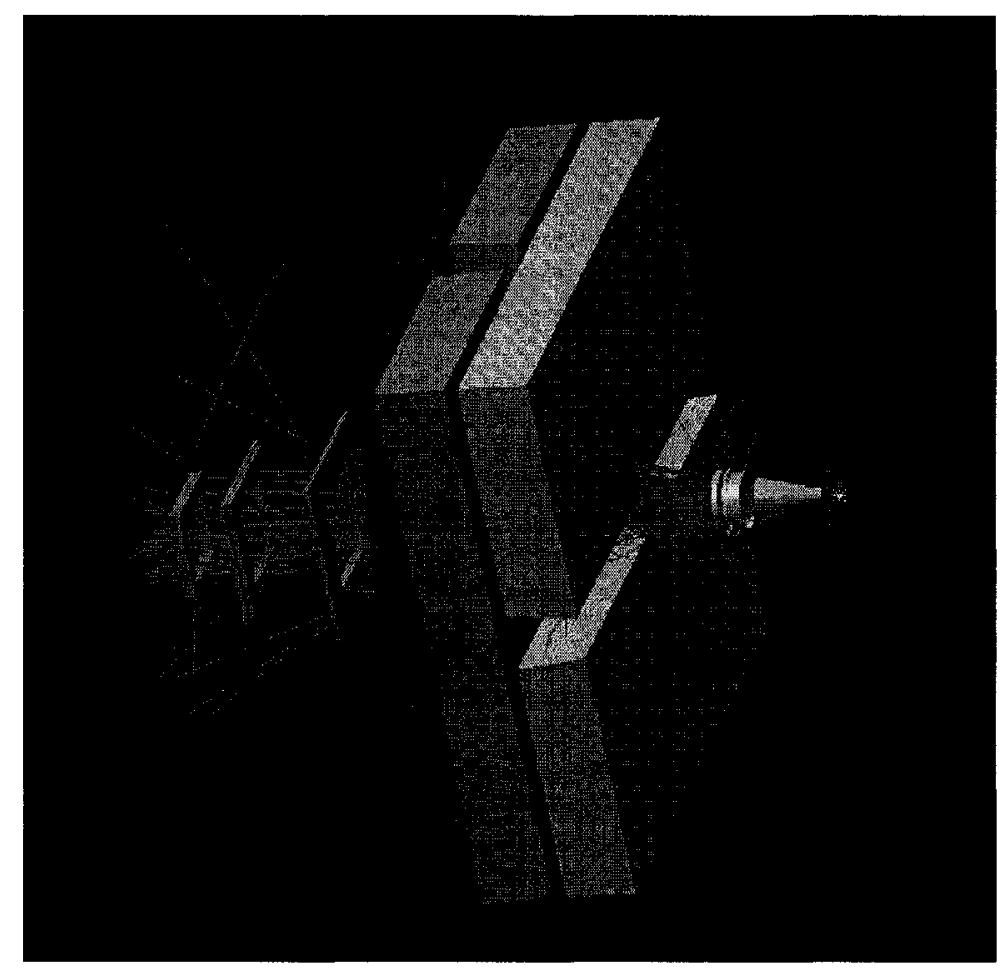

Figure 1.2: Model of an Elekta SL25 clinical linear accelerator for the $18 \mathrm{MeV}$ electron beam, $10 \times 10 \mathrm{~cm}^{2}$ field size, as produced by the BEAMnrc and the EGS_Windows 3D graphics application. Gray lines are electron tracks. 
To estimate statistical uncertainties of Monte Carlo results, BEAMnrc uses the history by history method. ${ }^{34}$ Quantities of interest can be calculated by averaging over a given set of histories. The uncertainty of the scored quantity $x$ is defined as

$$
s_{\langle x\rangle}^{2}=\frac{1}{N-1}\left(\frac{\sum_{i=1}^{N} x_{i}^{2}}{N}-\left(\frac{\sum_{i=1}^{N} x_{i}}{N}\right)^{2}\right)
$$

where $x_{i}$ is the quantity scored in statistically independent histories $i$ and $\mathrm{N}$ is the number of independent histories. The uncertainty is calculated at the end of the simulation by calculating $\sum_{i=1}^{N} x_{i}^{2}$ and $\sum_{i=1}^{N} x_{i}$ on the fly. To overcome the inefficiency of this method for a large number of quantities being scored, Sempau et al. ${ }^{35}$ outlined the algorithm that is now implemented in all NRC EGSnrc user-codes.

There are various of BEAMnrc input parameters, including EGSnrc parameters, that must be specified for each simulation. They are explained in Chapter 4.

\subsection{DOSXYZnrc}

DOSXYZnrc $^{36}$ is an EGSnrc user code to calculate the three dimensional (3D) absorbed radiation dose distributions in a rectangular voxel based phantom. It simulates particle transport in a Cartesian volume and scores the deposited energy in all the designated voxels. The voxel dimensions are independent in all three directions. The material and the density of each voxel can be specified individually. For statistical analysis, a history-by-history method ${ }^{34}$ is adopted in scoring the quantities of interest (e.g. deposited energy) in order to decide on the statistics of those quantities. This means scoring will be grouped by primary particles in the accelerator simulation when using phase space data.

There are several input source types for DOSXYZnrc. It could be a point

1.4. DOSXYZNRC 
source, a parallel incident beam, a beam characterization model or phase-space data generated by a previous BEAMnrc simulation. The last two source routines were mainly used in this project. DOSXYZnrc has the capability of dose component calculation, that is, distinguishing dose contribution by particle types or by component in the linac head based on LATCH bit filtering.

The output of a DOSXYZnrc calculation is a file that stores the calculated $3 \mathrm{D}$ dose distribution data and the corresponding dose uncertainties for all the voxels. The calculated dose values are normalized per incident history. The STATDOSE ${ }^{37}$ utility program, provided in the BEAMnrc software package, can be used to analyze the 3D dose files.

Particles can be recycled to reduce uncertainties in DOSXYZnrc calculations. Re-using particles may cause uncertainties to be underestimated if correlations between particles from the same initial history are not maintained. Recycling particles is essential when phase space data are sparse (fewer particles in the file than required for the simulation). Each particle in the phase space file can be recycled several times.

\subsection{BEAMDP code}

BEAMDP (BEAM Data Processor) is an interactive program to analyze the phasespace parameters of clinical electron and photon beams generated using BEAMnrc. All plots of parameters related to phase-space files (spectral distributions, fluence vs position, mean energy distributions, etc) for this project were generated using the BEAMDP utility.

BEAMDP is also used to derive the data required by a multiple-source model

\subsection{BEAMDP CODE}


for representation and reconstruction of the electron beam for use in Monte Carlo radiotherapy treatment planning. This functionality, because of its importance for this study, will be explained with more details in Chapter 3.

\subsection{Thesis Overview}

This introductory Chapter 1 gives some general ideas about Monte Carlo calculations and their use in radiotherapy, with further details about the computer packages used in the present work, particularly about EGSnrc in Section 1.2 and the BEAMnrc code in Section 1.3.

The Elekta SL25 linear accelerator is discussed in Chapter 2 which explains photon and electron modes of operation, geometry details of the linear accelerator, and determination of some initial parameters used in the Monte Carlo simulation of the treatment head. Experimental setup and measurements with electron beams performed at Institute for National Measurements Standards, National Research Council of Canada (INMS NRC) are presented and compared with the results of the simulation.

Chapter 3 explains the basis of multiple-source beam modeling. It describes source types, planar fluence and energy spectra used and the method used in the model creation. Changes in the BEAMDP code, which were done for this project, and their verification are described in this chapter.

A step-by-step description of the calculations used in obtaining the phasespace files, beam models, central-axis depth-dose and dose-profile curves are given in Chapter 4.

\subsection{THESIS OVERVIEW}


Chapter 5 discusses some relevant beam characteristics of electron and photon beams derived from the original phase-space files. These must be taken into consideration when building beam models, particularly, when deriving the number of significant sub-sources of the model and the number of energy radii.

Chapter 6 presents results of depth-dose calculations in water phantom obtained using multiple-source models versus ones from original phase-space files and comparison between original phase-space file and reconstructed phase-space file from beam models.

Finally, Chapter 7 describes summary of results and conclusions for multiplesource models. 


\section{Chapter 2}

\section{Elekta SL25 linear accelerator}

In this chapter the Elekta SL25 linear accelerator is described from the perspective of Monte Carlo modeling for photon and electron beams. Experiments performed at NRC to obtain central-axis depth-dose and dose-profiles for the electron beam are discussed. The results of simulations are compared against the experimental data for photon and electron beams individually, followed by a discussion of the influence of electron beam energy and incident electron spatial distribution on depth-dose curves.

\subsection{General introduction}

A clinical linear accelerator (linac) is the device most commonly used for external beam radiation treatments for cancer patients. The Elekta SL25 clinical linear accelerator is a high-energy and multimodality radiation treatment machine with asymmetric collimator jaws. It produces three photon beam qualities at 6,10 and $25 \mathrm{MV}$, and electron beams with five selectable energies at 4, 8, 12, 18 and $22 \mathrm{MeV}$. 
The Elekta SL25 linear accelerator accelerates electrons to kinetic energies from $4 \mathrm{MeV}$ to $25 \mathrm{MeV}$ within a special evacuated structure of the accelerator named the accelerating waveguide. Electrons follow straight trajectories through a cyclic sequence of low potential differences before entering a beam-bending system which consists of three in-line beam-bending electro-magnets. These magnets focus electrons to a spot approximately $1 \mathrm{~mm}$ in diameter, and direct the beam out of the waveguide to a small area on the x-ray target or electron scattering foil.

The Elekta linear accelerator can work in two modes: electron beam mode and photon beam mode. For the photon beam mode, electrons hit the thin x-ray target to produce bremsstrahlung photons. The target is made of a composite of high-Z materials, specifically, 90\% tungsten and 10\% rhenium. The same target is used for both, low- and high-energy x-ray beams. In the low-energy mode, a single iron filter, mounted in one of the five slots in a carousel, is used to produce a flat beam. The other four slots on the carousel are used in electron mode to position secondary scattering foils in the beam. The high-energy $x$-ray mode utilizes two iron flattening filters and an aluminum beam hardening filter to flatten the x-ray beam over the complete range of field sizes. A set of orthogonal photon jaws collimate the beam, allowing field sizes up to $40 \times 40 \mathrm{~cm}^{2}$.

For the electron beam mode, the primary electrons impinge on one of five available primary scattering foils. These are made from different thicknesses of tantalum. The appropriate scattering foil is automatically positioned in the beam for a particular nominal energy. The scattered broad beam is then flattened by one of four aluminum secondary scattering foils. The selected foil scatters the electron beam to give a flat and uniform beam. The x-ray jaws provide the first collimation for the broad electron beam. Settings are optimized for each applicator and energy. The

\subsection{GENERAL INTRODUCTION}


electron beams coming through the x-ray jaws are of uniform intensity distribution. Therefore the applicator has only to progressively collimate the beam using a set of three aperture plates without the use of any wall scatter. These aperture plates have decreasing dimensions downstream. The bottom aperture plate defines the treatment field size. This plate is removable and can be replaced with cutouts of irregular field shape. There are four standard applicators provided by the manufacturer, giving nominal field sizes of $6 \times 6,8 \times 8,10 \times 10$ and $20 \times 20 \mathrm{~cm}^{2}$. Other sizes are optionally available from the manufacturer.

A ceramic ionization chamber is used to monitor the linac output (Figure 2.1, page 27). It is essentially made of ceramic and aluminum with a central diameter of $90 \mathrm{~mm}$ so that the central area of the chamber is comprised of air and mylar film plates. For photon beams there is a backscatter plate located just downstream of the ion chamber to prevent backscatter radiation (electrons) entering the chamber.

Clinical photon and electron beams, even with the same nominal energies from linacs of the same model from the manufacturer, are generally treated as unique beams whose dosimetric characteristics should be simulated (or measured) individually because of differences in the beam forming parts in linacs of the same model. Each of the beams listed in Table 2.1 were simulated individually for combinations of energies and field sizes. A collection of BEAMnrc input files for electron beams of the Elekta SL25 were originally created by Blake Walters and David W.O. Rogers in 2003. Experimental data for electron beams that are used in this project are electron beam commissioning data from 2003 collected by Malcolm McEwen at NRC, and new data obtained in May 2005 in conjunction with Malcolm McEwen. BEAMnrc input files for photon beams were obtained from Elena Tonkopi and Iwan Kawrakow at NRC together with experimental data collected by Malcolm McEwen during 2005.

\subsection{GENERAL INTRODUCTION}


Table 2.1: BEAMnrc photon and electron beams simulation for the NRC Elekta SL25 treatment head

\begin{tabular}{ll}
\hline \hline $\begin{array}{l}\text { Photons } \\
10 \times 10 \mathrm{~cm}^{2}, 40 \times 40 \mathrm{~cm}^{2}\end{array}$ & $\begin{array}{l}\text { Electrons } \\
10 \times 10 \mathrm{~cm}^{2}, 20 \times 20 \mathrm{~cm}^{2}, 40 \times 40 \mathrm{~cm}^{2}\end{array}$ \\
\hline $4 \mathrm{MV}$ & $4 \mathrm{MeV}$ \\
$10 \mathrm{MV}$ & $8 \mathrm{MeV}$ \\
$25 \mathrm{MV}$ & $12 \mathrm{MeV}$ \\
& $18 \mathrm{MeV}$ \\
& $22 \mathrm{MeV}$ \\
\hline \hline
\end{tabular}

\subsection{Monte Carlo modeling of Elekta SL25 linac}

To simulate a medical linear accelerator, very detailed information about each component in the linac's treatment head, including the material, geometrical shape, size, and position is required. Some components may be in the beam path permanently, while others may be optional. These components were generally referred as "patient independent" and "patient dependent" components respectively, as shown in Table 2.2. Monte Carlo simulation of radiation transport inside the treatment head begins within the various patient-independent components. This is also termed the phase-space simulation. The treatment head components include the target/exit window, scattering foils, primary collimator, flattening filters, transmission chamber, mirror and mylar screen. It is also possible to include other, patient dependent components, such as applicator and bolus and other similar modifiers in the patient simulation code.

Unfortunately, the specifications of a linac treatment head are not easily accessible, mostly because of proprietary nature of these machines. In addition to this,

\subsection{MONTE CARLO MODELING OF ELEKTA SL25 LINAC}


Table 2.2: Generic components in the treatment head for photon and electron beam modes.

\begin{tabular}{lll}
\hline \hline Description & Photon beam mode & Electrons beam mode \\
\hline Patient independent & Exit window & Exit window \\
components & Target & Primary scattering foil \\
& Primary collimator & Primary collimator \\
& Flattening filter & Secondary scattering foil \\
& Monitor & Monitor \\
& Backscatter plate & \\
& Mirror & Mirror \\
& Mylar screen & Mylar screen \\
\hline Patient dependent & Asymmetric jaws / MLC & Jaws (fixed for each applicator) \\
& Wedge,blocks,graticule & Applicator \\
components & Bolus & Bolus \\
\hline \hline
\end{tabular}


linacs specifications, although generally very accurate and detailed, may not contain all the information required to build a simulation model, and additional measurements may be needed. Specification may also be incomplete due to linac upgrades, or due to large uncertainties of some important parameters for the simulation, for example, electron beam energy and incident electron distribution.

Modeling of electrons prior to exiting the vacuum tube is usually not done as part of Monte Carlo simulation. One has to make some assumptions about the character of the initial electron beam. These assumptions have a strong influence on results and play a very important role in every Monte Carlo simulation of linear accelerators. The simulation results are very sensitive to these details of the model. It is an iterative process of adjusting the various parameters of the source and treatment head geometry to fit the measurements which requires Monte Carlo expertise and an understanding of photon and electron beams characteristics.

The model consists of a series of component modules, as shown generically in the schematic diagram of the Elekta SL25, Figure 2.1. Each component is contained between the two planes which are perpendicular to the z-axis and which can not overlap (overlapping causes an error message in the BEAMnrc code). The BEAMnrc code models the therapy source with the z-axis taken as the beam-axis and usually the origin is defined as the center of the beam as it exits from the accelerator vacuum window for electron beams, or hits the photon beam target for photon beams (it was noticed, late in this project, that the vacuum window has not been simulated for the photon beams).

In general, there are four steps to run a BEAMnrc simulation:

1. Specify the accelerator, i.e. all CMs used in the linac simulation and their order.

\subsection{MONTE CARLO MODELING OF ELEKTA SL25 LINAC}


This information is contained in a 'module' definition file.

2. Build and compile all software components.

3. Define input data, i.e. all parameters, geometry details and materials for each CM. This information is contained in an 'egsinp' input file.

4. Run the program and analyze. The program may be executed in either batch mode or interactive mode.

\subsubsection{Geometry details and schematic diagram}

The Elekta SL25 medical linear accelerator has been modeled and implemented for 4, 10 and $25 \mathrm{MV}$ photon beams and for 4,8,12,18 and $22 \mathrm{MeV}$ electron beams with various field sizes (Table 2.1) throughout this work.

Figure 2.1 shows the model of the Elekta SL25 treatment head for the photon and electron beams. When modeling the Elekta SL25 linac head in the photon mode, the following components are always present along the beam path: target, flattening filters, monitor chamber, backscatter plate, mirror, secondary collimator (photon jaws) and mylar screen and for the electron beams: vacuum exit window, scattering foils, collimator, monitor chamber, mirror, photon jaws, mylar screen and applicators.

The Elekta SL25 linac photon target is modeled with the SLABS component module. The vacuum exit window is modeled with the SLABS CM by a layer of nickel. The primary collimators, primary scattering foil and monitor chamber are modeled using the CONESTAK CM due to its cylindrical structure. The flattening filter is modeled by the FLATFILT CM, which can be used inside a conical collimator.

\subsection{MONTE CARLO MODELING OF ELEKTA SL25 LINAC}



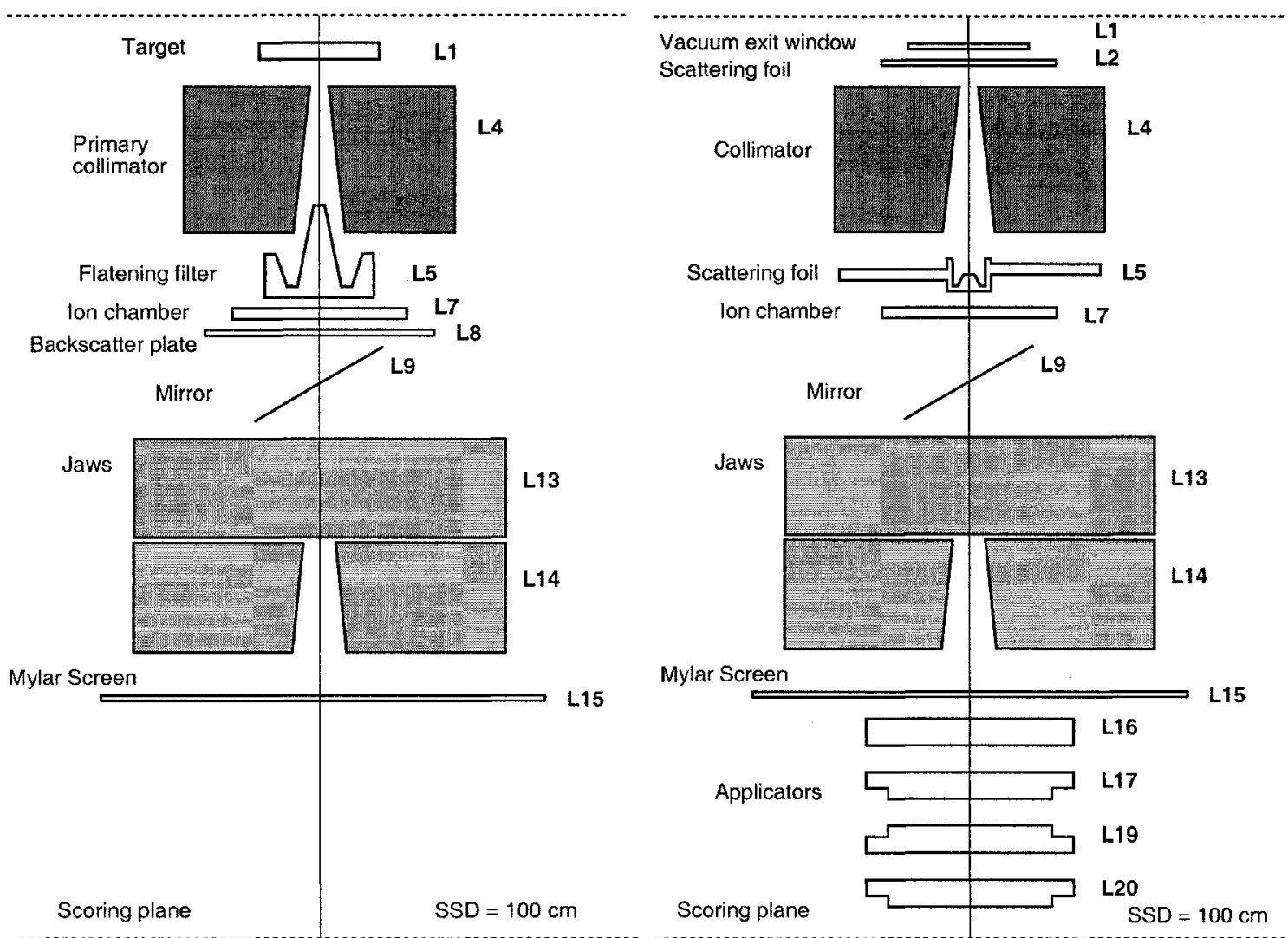

Figure 2.1: Schematic diagram for Elekta SL25 accelerator showing relative positions of accelerator components for photon mode of operation (left) and electron mode of operation (right). LATCH bits associated with each component are shown on the right. The scoring plane is located at $\mathrm{SSD}=100 \mathrm{~cm}$. This picture is not to scale. 
The MIRROR CM is used to model the mirror in the linac head. The upper jaws are modeled by the JAWS CM, and the applicators by the APPLICAT CM. The component modules (CM) used in the BEAMnrc models are described in Table 2.3. An important parameter for each $\mathrm{CM}$ is its associated LATCH bit. Using LATCH bits one can keep track of each particle's history and classify the origin of a particle. The LATCH variable contains the region number (from 1 to 23) where the particle has been, has interacted, or was created if it is a secondary particle (bits 24 to 28). The origin of a bremsstrahlung photon is considered to be the region where it is created or scattered in the case of Compton or coherent scattering. A charged particle's origin is considered to be the last non-air region it has been to before it reaches the scoring plane. This is a very important parameter for multiple-source models and it will be explained in Chapter 3.

The result of a simulation is a phase-space file. Throughout this work the phase-space files are scored at $\mathrm{SSD}=100 \mathrm{~cm}$ for all simulated beams in Table 2.1. These phase-space files are used as an input for:

- Dose calculation, which were referred to as dose calculation from the phasespace file

- Creation of a multiple-source model

Comparison plots, analysis and presentation of results are not given for all simulations listed in the table, but mostly for the $18 \mathrm{MeV}$ electron beams and $25 \mathrm{MV}$ photon beams as these are the more difficult ones to obtain good agreement with experiment. 
Table 2.3: Component Modules (CM) in the treatment head of Elekta SL25 .

\begin{tabular}{|c|c|}
\hline CM & Description/use in modeling Elekta SL25 linac \\
\hline SLABS & $\begin{array}{l}\text { Models parallel slabs of arbitrary materials and thickness in the } \\
\text { X-Y plane. The outer boundary is square. Used to model x-ray } \\
\text { target, electron exit window and mylar screen with the slab of air. }\end{array}$ \\
\hline CONESTAK & $\begin{array}{l}\text { Models a series of stacked truncated cones which can be cylinders. } \\
\text { Used to model the primary scattering foil and ion chamber. }\end{array}$ \\
\hline FLATFILT & $\begin{array}{l}\text { Generalization of CONESTAK component module for an arbitrary number } \\
\text { of truncated conical sections on each layer. The most complex CM. } \\
\text { Used to model the flattening filters, primary collimator and secondary } \\
\text { scattering foil. }\end{array}$ \\
\hline CHAMBER & $\begin{array}{l}\text { Models an ionization chamber and other structures with cylindrical } \\
\text { symmetry centered on the beam axis. For a monitor chamber both the } \\
\text { front and back walls may have several layers of different materials. } \\
\text { Used to model a cylindrical water phantom for depth-dose calculations. }\end{array}$ \\
\hline MIRROR & $\begin{array}{l}\text { Models a mirror in the accelerator. Can have several layers of } \\
\text { arbitrary thickness and materials. Square boundary with arbitrary } \\
\text { position in the } \mathrm{x} \text { direction. }\end{array}$ \\
\hline JAWS & $\begin{array}{l}\text { Models a pair of opposing flat surfaces which are perpendicular to } \\
\text { the } \mathrm{x} \text { or } \mathrm{y} \text { axis. The jaw surfaces may be at an arbitrary angle with } \\
\text { respect to the } \mathrm{z} \text { axis. The outer boundary is square. Used to model } \\
\text { the jaws of Elekta SL25 linear accelerator. }\end{array}$ \\
\hline APPLICAT & $\begin{array}{l}\text { Models a set of rectangular scrapers defined by the outer region of } \\
\text { two concentric rectangles with inner region being air. Used to model } \\
\text { an applicator. }\end{array}$ \\
\hline
\end{tabular}




\subsection{Water phantom measurements}

The central-axis depth-dose and dose-profiles in the water phantom were measured, in conjunction with Malcolm McEwen at $\mathrm{NRC}$, for 4,8,12,18,22 MeV electron beams from an Elekta SL25 linac. A dosimeter was mounted on a Multidata Systems International Corp three-dimensional scanning system which included the water tank and the real-time dosimetry software, RTD scanner, for data acquisition. The scanner was computer controlled with a placement precision of about $1 \mathrm{~mm}$ (Figure 2.2).

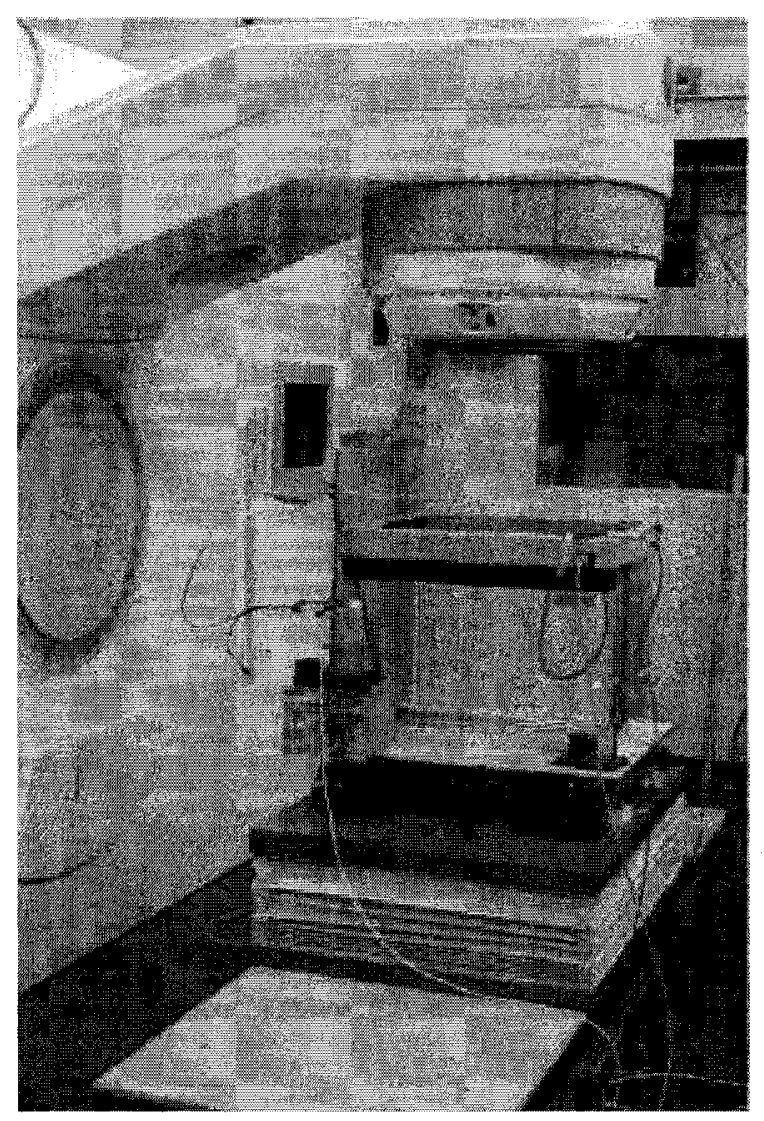

Figure 2.2: Phantom setup for a dosimetry measurements in water with the Scanditronix water phantom tank and dosimetry scanner

\subsection{WATER PHANTOM MEASUREMENTS}


The water tank was filled with water and positioned with the source to water surface distance (SSD) $100 \mathrm{~cm}$. A vertical beam was used throughout.

Data was acquired in the continuous scanning mode using the following dosimeters:

1. Diode - Scanditronix EFD (electron field detector) diode manufactured by Scanditronix-Wellhofer, now owned by IBA

2. Thimble chamber-Exradin A16 micro-chamber manufactured by Standard Imaging

3. Parallel-plate chamber-NACP-02 manufactured by Scanditronix-Wellhofer, now owned by IBA

Three chambers were used in the measurements, because of known significant differences found in the buildup dose regions ${ }^{38,39}$ among parallel-plate, cylindrical ion chambers and diode detectors. It is recommended that buildup doses be carefully measured by parallel-plate chamber or extrapolation chamber. ${ }^{40}$ Diode detectors, which give very good spatial resolution, may also be considered but should be crossreferenced with other detectors.

Measurements were done for each energy listed in Table 2.1 for $10 \times 10 \mathrm{~cm}^{2}$, $20 \times 20 \mathrm{~cm}^{2}$ and $40 \times 40 \mathrm{~cm}^{2}$ field sizes. At each energy, four sets of ionization measurements are performed: a central-axis depth-dose ionization scan, and three dose-profile scans at different depths. The total scan was up to $15 \mathrm{~cm}$ depth for depth-ionization scan for electron beams, and $40 \mathrm{~cm}$ (20 cm each side of the central axis), for doseprofiles. The step size was $1 \mathrm{~mm}$ and the integration time at each point was 0.4 second. This combination of parameters resulted in a total time for each scan of

\subsection{WATER PHANTOM MEASUREMENTS}


between three and four minutes.

Depth-ionization curves for ion chambers were converted to depth-dose curves by using a 9-parameter equation for the necessary Spencer-Attix water to air stopping power ratio as a function of $R_{50}$ and depth given by Burns et al. ${ }^{41}$

\subsection{Elekta SL25 photon beams}

It was already mentioned that some components of the treatment head that interact with the photon beam have a very strong influence on the characteristics of the beam and that the incident electron beam's energy and spatial distributions are key parameters in the Monte Carlo simulation. The manufacturer's specifications for the electron beam's energy distribution can only be taken as a first estimate in the iterative process of adjusting the parameters of the beam in order to get better agreement with experiment.

It is very well known that the key parameters in the simulation of a linear accelerator are the mean energy and focal spot size of the electron beam incident on the exit window. ${ }^{42,43}$

Sheikh-Bagheri and Rogers ${ }^{42}$ found that the incident electron beam radial intensity distribution influences the off-axis ratios. The larger the width of the electronbeam radial intensity distribution, the relatively more intense the photon beam on the central axis. However, the central-axis depth-dose values are quite insensitive.

Elena Tonkopi et al. ${ }^{44}$ enhanced the "Parallel circular beam with Gaussian radial distribution" source (ISOURC $=19$ in BEAMnrc) by allowing for an angular distribution of incident electrons in order to match the experimental results for the

\subsection{ELEKTA SL25 PHOTON BEAMS}


$25 \mathrm{MV}$ photon beam from the Elekta SL25 linear accelerator. Initially, they had good agreement for lower photon beam energies (Figures 2.3 and 2.4 shows $6 \mathrm{MV}$ and 10MV photon beams), but there were discrepancies in the buildup region for the 25 MV photon beam. Figure 2.5 shows the comparison of the Monte Carlo calculated

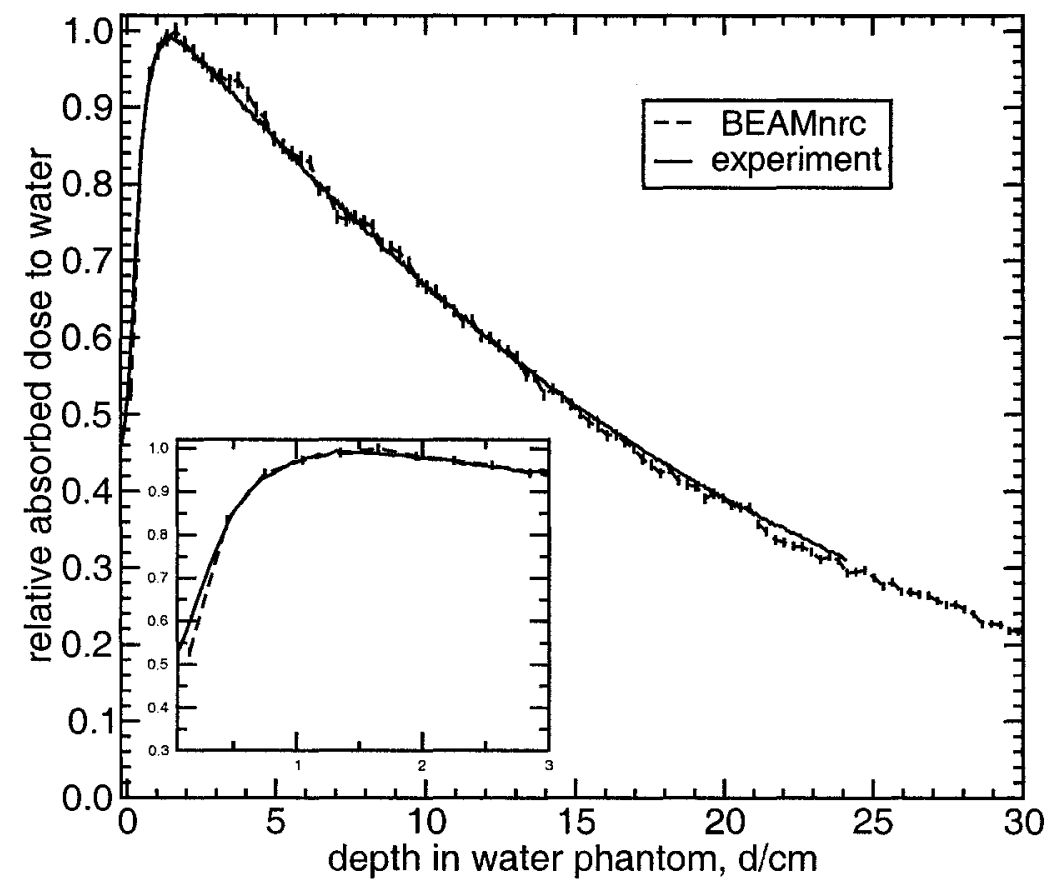

Figure 2.3: Comparison of measured and calculated central-axis depth-dose curves for the Elekta SL25 $6 \mathrm{MV}$ photon beam. The simulation curve is with the angular distribution of incident electron beam. The field size is $10 \times 10 \mathrm{~cm}^{2}$ at an SSD of 100 $\mathrm{cm}$.

values of central-axis depth-dose curves with/without the angular distribution of the incident electrons for the $25 \mathrm{MV}$ photon beam. The discrepancy in the build-up region is evident in the no-angular-distribution case. However, the agreement between calculation and measurements for the other case is generally better than $0.5 \%$. All curves are normalized to the value at $d_{\max }$. The results of our simulations using the input files developed at NRC, and the investigations performed at NRC by Elena Tonkopi et al. ${ }^{44}$ based on the same BEAMnrc input files, both imply that these

\subsection{ELEKTA SL25 PHOTON BEAMS}




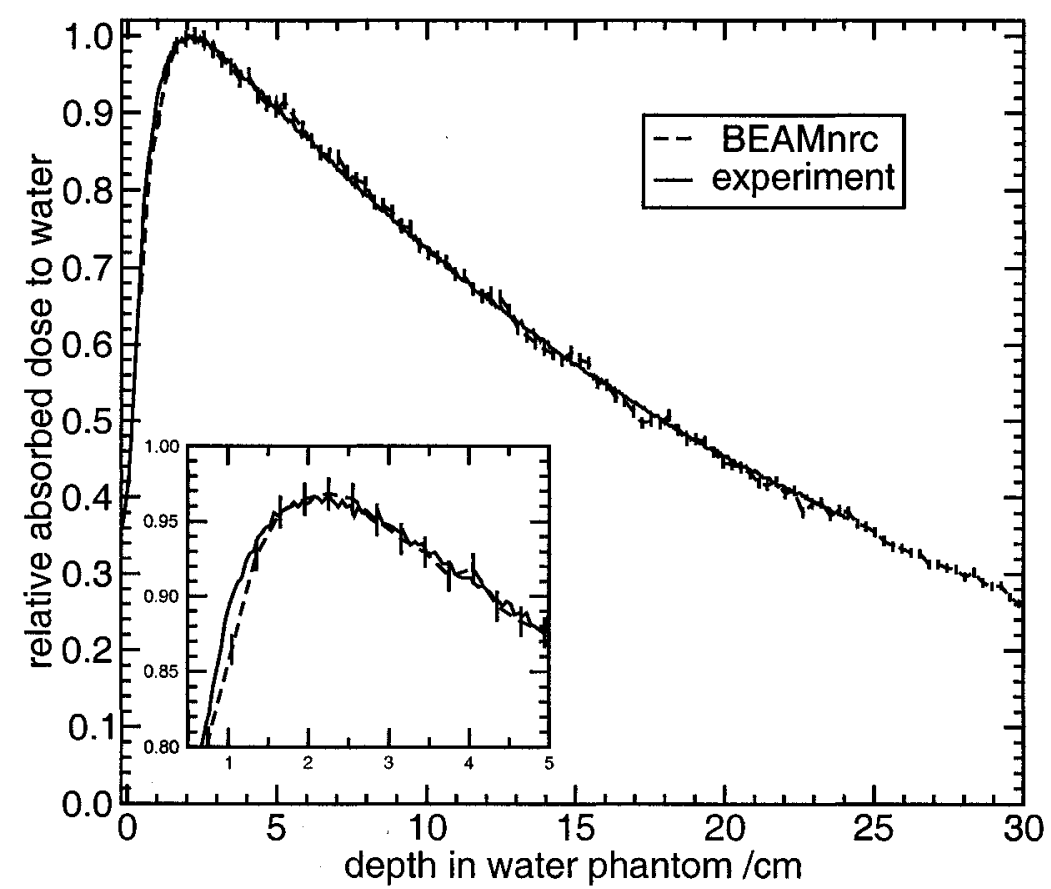

Figure 2.4: Comparison of measured and calculated central-axis depth-dose curves for the Elekta SL25 $10 \mathrm{MV}$ photon beam. The simulation curves are with an angular distribution of the incident electron beam. The field size is $10 \times 10 \mathrm{~cm}^{2}$ at an SSD of $100 \mathrm{~cm}$.

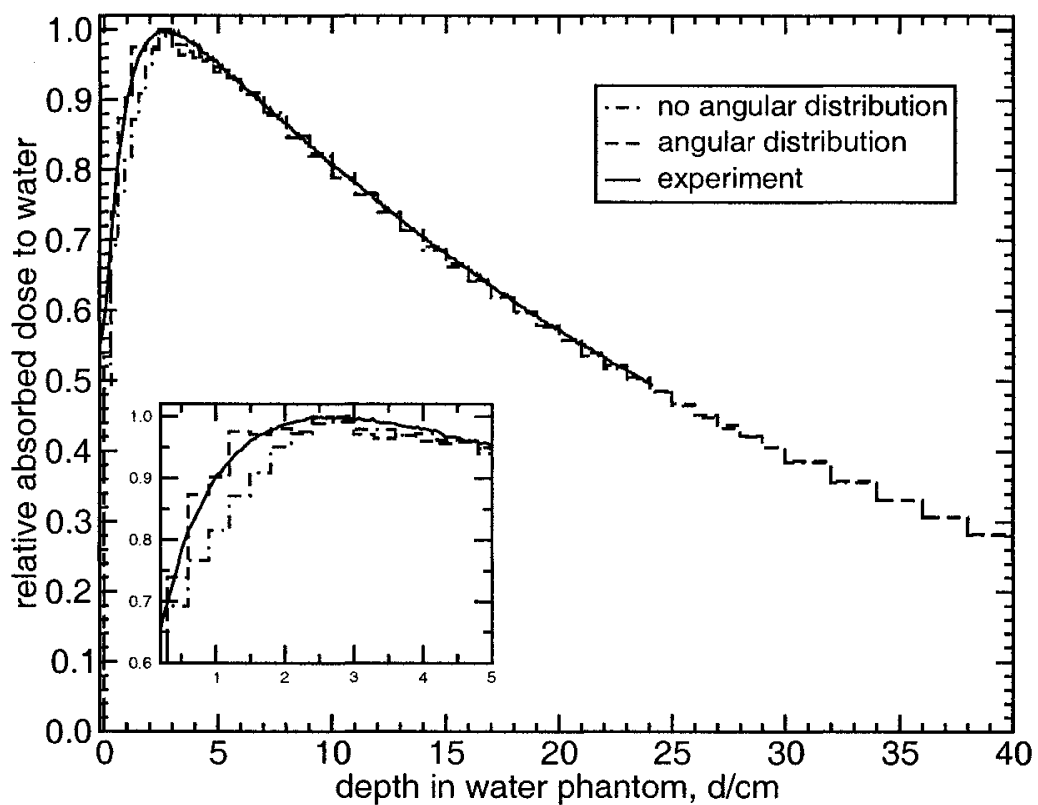

Figure 2.5: Comparison of measured and calculated central-axis depth-dose curves for the Elekta SL25 $25 \mathrm{MV}$ photon beam. The simulation curves are with/without the angular distribution of incident electron beam. The field size is $40 \times 40 \mathrm{~cm}^{2}$ at an SSD of $100 \mathrm{~cm}$.

\subsection{ELEKTA SL25 PHOTON BEAMS}


are adequate BEAMnrc models and phase-space files upon which to build multiplesource models for the 6,10 and $25 \mathrm{MV}$ photon beam energies of the Elekta SL25 linear accelerator.

\subsection{Elekta SL25 electron beams}

The procedure for simulating the electron beam treatment head is similar to that of photon beams, with different critical parameters than those for x-rays. However, electron distributions are very sensitive to all the materials in the beam, especially the scattering foils which are designed to flatten the beam with minimal bremsstrahlung contamination. Accurate geometric descriptions are also required for electron beams.

The incident electron energy is the primary tuning parameter for electron beam simulations. A good match for the depth-dose curve requires an accurate mean energy, and a properly broadened peak in the initial spectrum (Figures 2.6 and 2.7) provide a better agreement for the slope of the depth-dose curves (Faddegon et al. ${ }^{45}$ ). In electron beam calculations the incident electron beam energy is iteratively adjusted to give the measured values of $R_{50}$. A change of $0.2 \mathrm{MeV}$ in the electron energy corresponds to about a $1 \mathrm{~mm}$ change in beam range, according to equation 2.1 for water

$$
\Delta E_{0}=2.33 * \Delta R_{50}
$$

where $\Delta E_{0}$ is in $\mathrm{MeV}$ and $\Delta R_{50}$ in $\mathrm{cm}$.

A monoenergetic incident electron beam with Gaussian radial distribution were used to match the experimental values of $R_{50}$. Table 2.4 presents the values

of nominal and incident energies and $R_{50}$ derived using this method. The Gaussian energy distribution spectra with iteratively adjusted FWHM for incident electron

\subsection{ELEKTA SL25 ELECTRON BEAMS}


Table 2.4: Details of the incident electron energies for Elekta SL25. The nominal beam energy specified by the manufacturer is given by $E_{\text {nominal }}$ and incident electron energy by $E_{i}$. Also shown are the measured beam quality specifier $R_{50}$, depth of dose maximum $d_{\max }$ and the FWHM values for initial energy spectra.

\begin{tabular}{lcccc}
\hline \hline$E_{\text {nominal }}(\mathrm{MeV})$ & $E_{i}(\mathrm{MeV})$ & $R_{50}(\mathrm{~cm})$ & $d_{\text {max }}(\mathrm{cm})$ & $F W H M(\mathrm{MeV})$ \\
\hline 4 & 5.15 & 1.87 & 0.84 & 1.0 \\
8 & 8.87 & 3.18 & 1.63 & 1.7 \\
12 & 12.81 & 4.80 & 2.60 & 2.4 \\
18 & 18.78 & 7.10 & 3.20 & 2.8 \\
22 & 23.70 & 8.90 & 1.40 & 2.2 \\
\hline \hline
\end{tabular}

beam were used to get a good match for the slope of the depth-dose curves, without affecting the $R_{50}$ values (Figure 2.6 )

Depth-dose curves and dose-profiles through the treatment volume, where the energy deposition from primary electrons dominates the dose distribution, are insensitive to the angular distribution and angle of incidence of electrons at the exit window of the accelerator, where the treatment head simulation begins (Bjork et al. ${ }^{46}$ ).

The result of the simulation of electron beams showed a good agreement for all energies for depths greater than $d_{\max }$ but there is disagreement in the buildup region, as shown in Figure 2.10, where the calculations underestimate the measured doses. Many parameters available in BEAMnrc were adjusted to try to get good agreement in the buildup region. Any change in the thickness of the electron exit window or primary scattering foil, showed a strong affect on the bremsstrahlung tail where good agreement had already been achieved. Including an angular distribution of the incident electrons did not improve the buildup region at all. Changing the

\subsection{ELEKTA SL25 ELECTRON BEAMS}




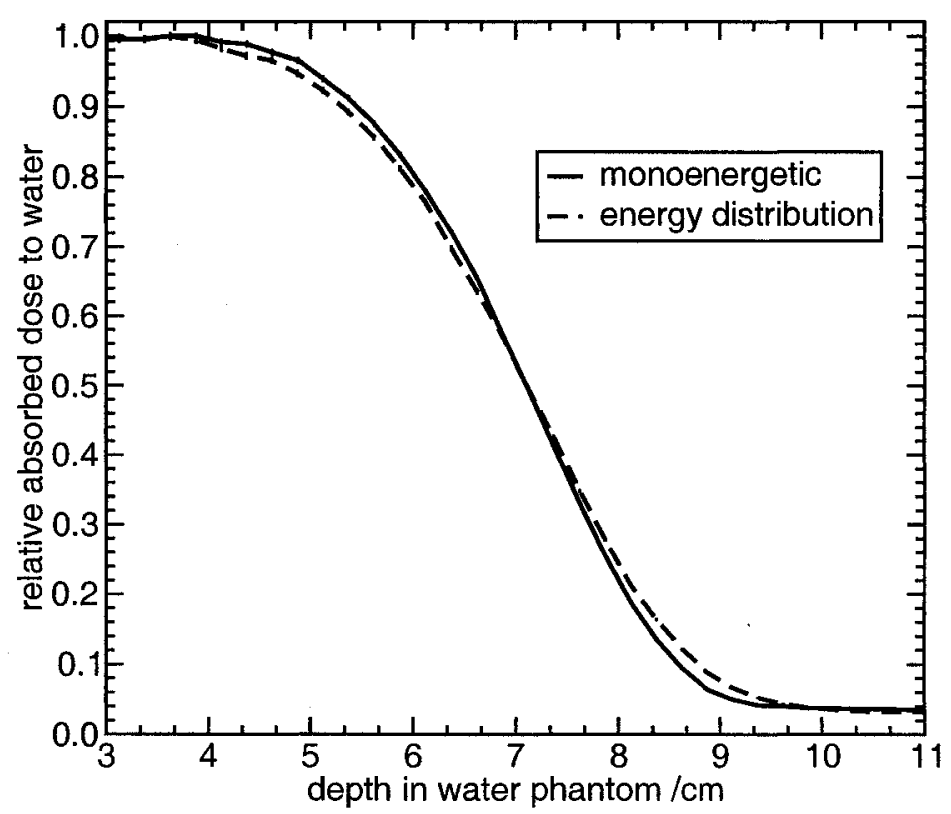

Figure 2.6: Effect of incident electron energy distribution on depth-dose curve for the Elekta SL25 $18 \mathrm{MeV}$ electron beam. The slope of calculated depth-dose curves for monoenergetic and Gaussian energy distribution spectra of incident electrons. FWHM used is $2.8 \mathrm{MeV}$. The field size is $10 \times 10 \mathrm{~cm}^{2}$ at an SSD of $100 \mathrm{~cm}$.

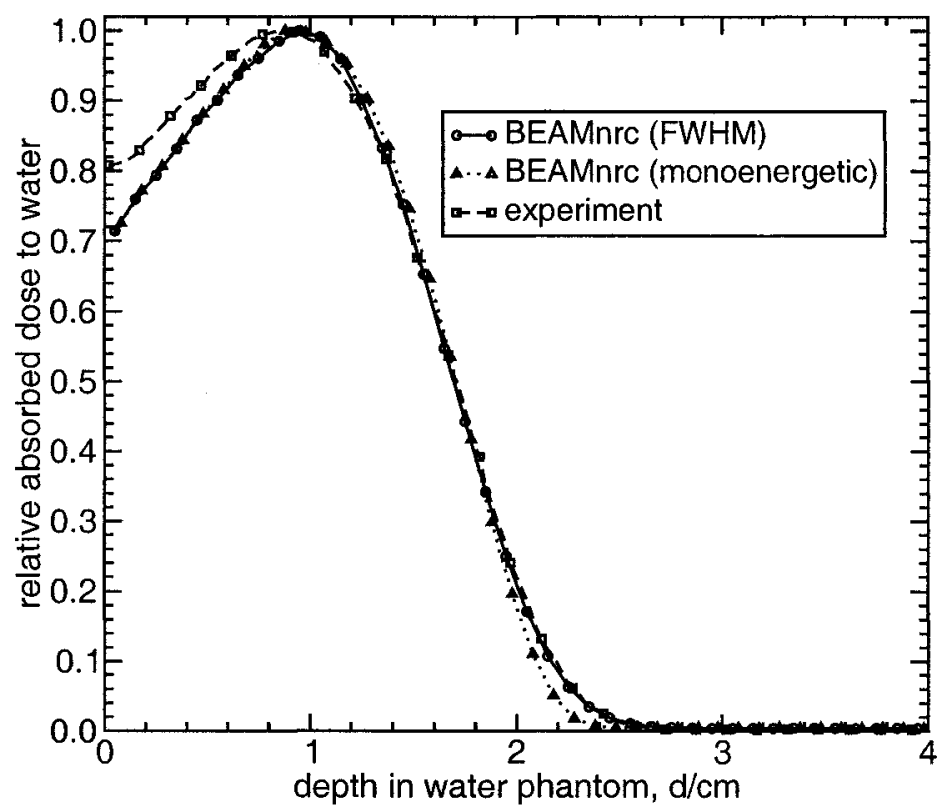

Figure 2.7: Comparison of measured and calculated central-axis depth-dose curves for the Elekta SL25 $4 \mathrm{MeV}$ electron beam. The slope of the depth-dose curve for calculated beam with monoenergetic incident electrons was adjusted by changing the FWHM of the Gaussian energy distribution spectra of the incident electrons. The field size is $10 \times 10 \mathrm{~cm}^{2}$ at an SSD of $100 \mathrm{~cm}$.

\subsection{ELEKTA SL25 ELECTRON BEAMS}




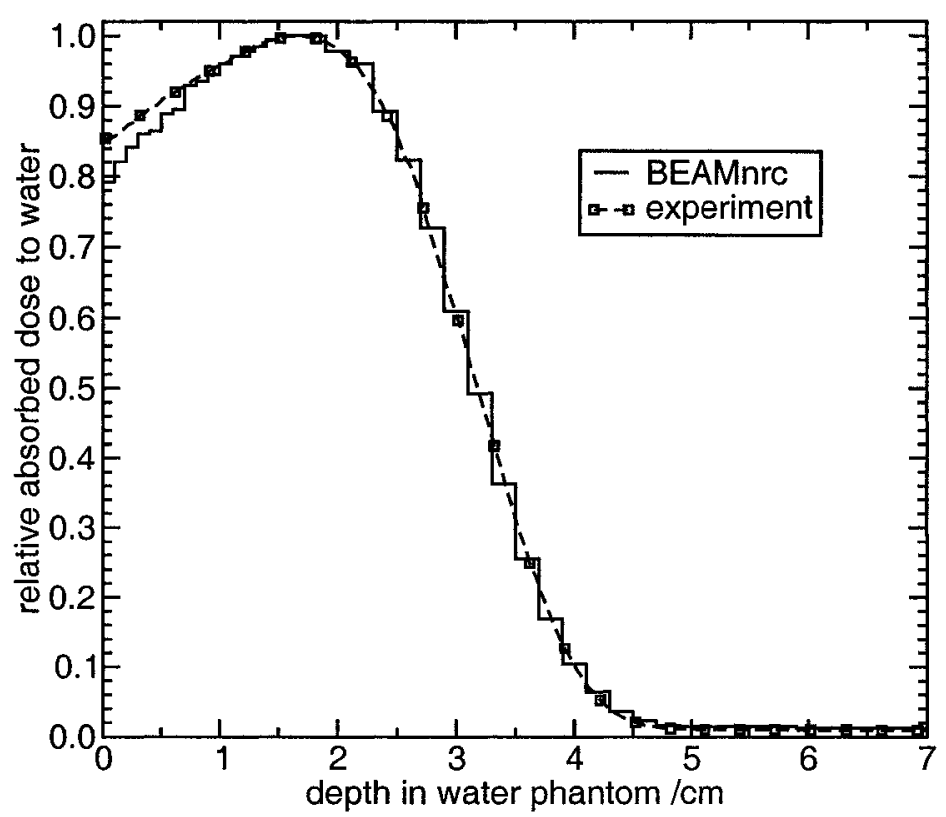

Figure 2.8: Comparison of measured and calculated central-axis depth-dose curves for the Elekta SL25 $8 \mathrm{MeV}$ electron beam. The slope was iteratively adjusted by changing the FWHM of the Gaussian energy distribution spectra of the incident electrons. The FWHM used for this energy is $1.7 \mathrm{MeV}$ (Table 2.4). The field size is $10 \times 10 \mathrm{~cm}^{2}$ at an SSD of $100 \mathrm{~cm}$.

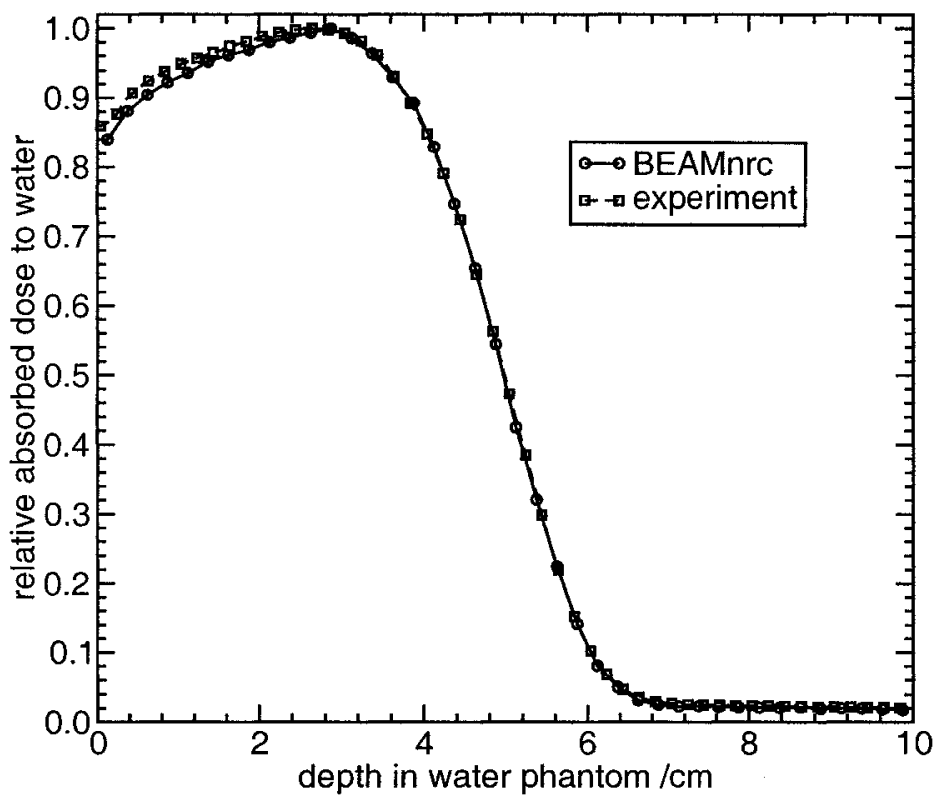

Figure 2.9: Comparison of measured and calculated central-axis depth-dose curves for the Elekta SL25 $12 \mathrm{MeV}$ electron beam. The field size is $10 \times 10 \mathrm{~cm}^{2}$ at an SSD of $100 \mathrm{~cm}$. 


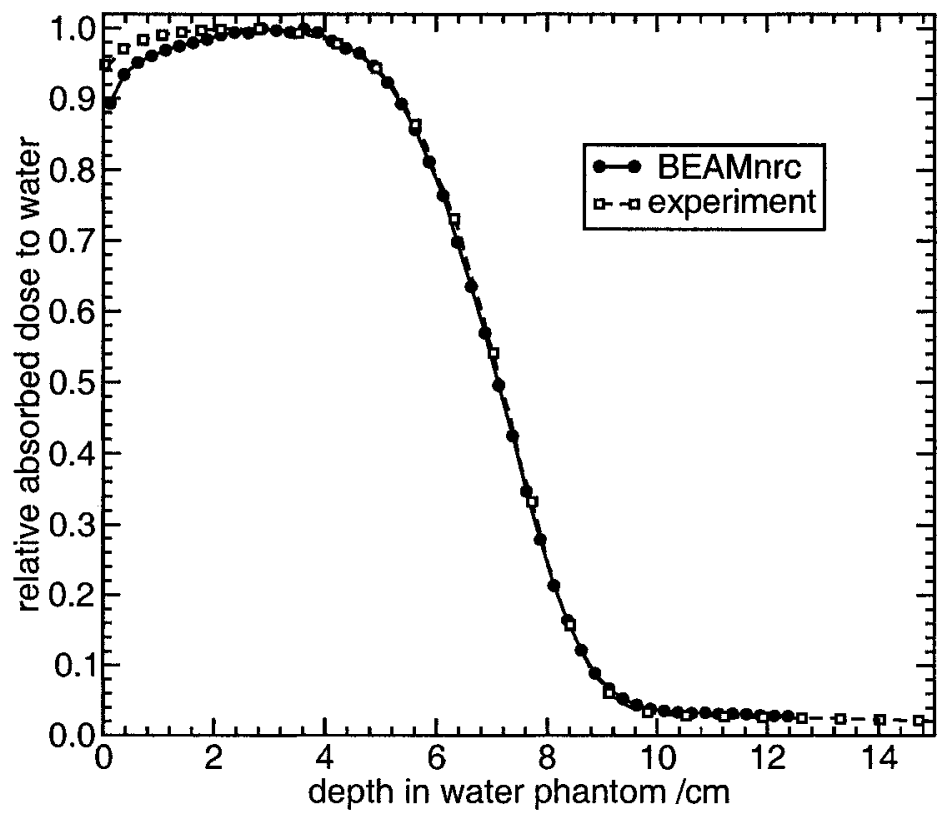

Figure 2.10: Comparison of measured and calculated central-axis depth-dose curves for the Elekta SL25 $18 \mathrm{MeV}$ electron beam. The field size is $10 \times 10 \mathrm{~cm}^{2}$ at an SSD of $100 \mathrm{~cm}$.

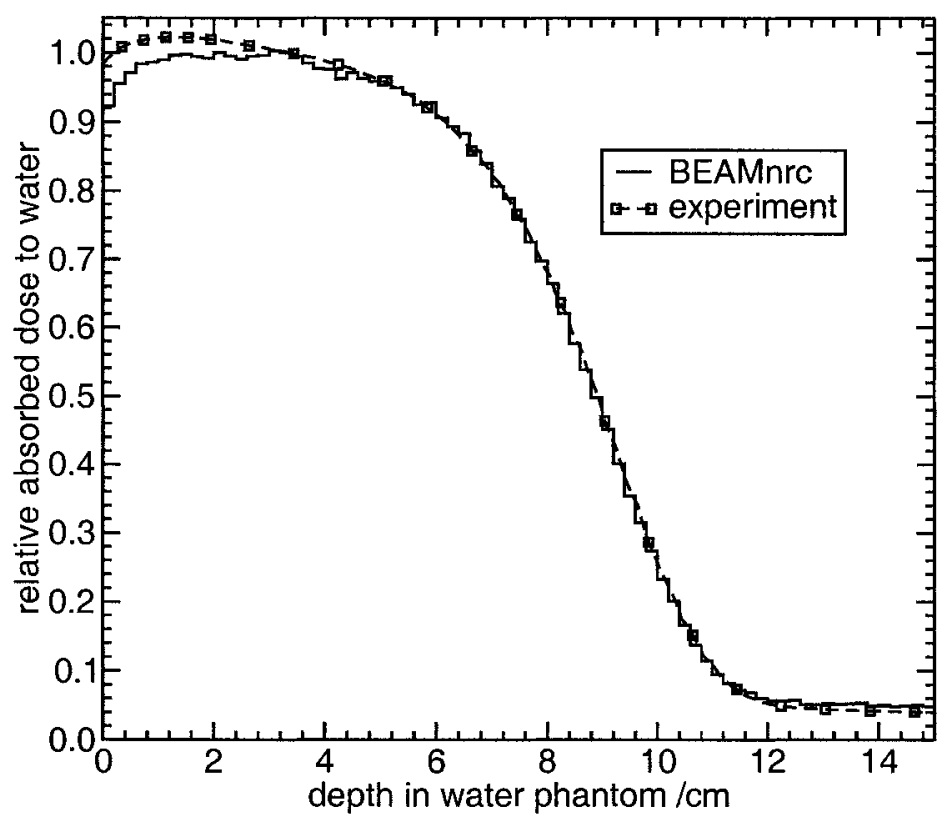

Figure 2.11: Comparison of measured and calculated central-axis depth-dose curves for the Elekta SL25 $22 \mathrm{MeV}$ electron beam. The slope were iteratively adjusted by changing the FWHM of the Gaussian energy distribution spectra of the incident electron. The FWHM used for this energy is $2.2 \mathrm{MeV}$ (Table 2.4). The field size is $10 \times 10 \mathrm{~cm}^{2}$ at an SSD of $100 \mathrm{~cm}$.

\subsection{ELEKTA SL25 ELECTRON BEAMS}


boundary tolerance showed only a strong effect around $d_{\max }$ towards greater dose depths but no effect in buildup region.

There are other studies that show the same inconsistency in the buildup region for Elekta SL25 electron beams. Deasy et al. ${ }^{47}$ performed measurements of the incident electron energy, and they found that the Elekta SL25 have highly variable spectra with varied distributions and that the energy widths of the peak region are so wide that there are substantial electron components well below the peak. Bjork et al. ${ }^{48}$ found that a more complex initial energy spectrum would improve the agreement between simulated and measured depth-dose curves.

There remains an inconsistency in the buildup region of the depth-dose curves for electron beams of Elekta SL25 for all energies. The primary goal of this project is to develop beam models of these beams and this will be done based on the best input files currently available. In future, if the reason for the discrepancy is found, it will be possible to quickly generate an improved beam model for all electron energies of the Elekta SL25 linear accelerator. 


\section{Chapter 3}

\section{Multiple-source model}

This chapter describes a multiple-source model for characterization of electron and photon beams from the Elekta SL25 clinical linear accelerator.

The program, BEAMDP ${ }^{1}$ and associated MORTRAN replacement macros have been modified for this project, and used to analyze the phase-space data, create multiple-source models and reconstruct the phase-space parameters of the modeled beams for either dose calculation or phase-space reconstruction itself. Methods to create a multiple-source model and beam reconstruction are explained.

Modifications of the original BEAMDP code include the separation of spatial binning inside and outside the treatment field to allow better resolution inside and around the edge of the treatment field and multiple-energy spectra to handle the case when the mean energy varies significantly in different spatial regions of the beam. Some results obtained with the original BEAMDP code versus modified code are presented. 


\subsection{About multiple-source models}

Multiple-source models ${ }^{2}$ are characterizations of electron and photon beams from a clinical accelerator. It is based on the observation that particles from different components of an accelerator have significantly different energy, angular and spatial distributions, while the particles from the same component have very similar characteristics in terms of energies and incident directions.

Particles coming from different parts of an accelerator may be treated as if they are coming from different sub-sources. Each sub-source represents one or more components in the linear accelerator geometry. Its intensity, type and geometrical dimensions are determined by the component.

A multiple-source model is built from the phase space file of a full Monte Carlo simulation. The program BEAMDP is used to analyze the phase-space data and create the model. The relative intensity, field planar fluence distribution and energy distribution are obtained for each of the sub-sources from the phase-space data. One sub-source radiates only one type of particle, photons, electrons or positrons. Each particle in the phase-space file contains a parameter, LATCH, which records detailed information about the particle history, such as where the particle interacts, where it is created if it is a secondary particle, or whether there is a bremsstrahlung photon involved in the particle's history.

Scattering in the air is also recorded in a particle's LATCH bits, in other words, included in the original phase-space file. In phase-space re-construction from the source model, scattering in the air is modeled by creating a small perturbation of the incident direction of charged particles by random sampling from the simulated angular probability distribution (see section 3.6).

\subsection{ABOUT MULTIPLE-SOURCE MODELS}




\subsubsection{Particle Origin}

Particles in the phase-space file are classified by their charge and LATCH bit. There is no sequence history for LATCH bits. A particle may interact in a region and go backwards and hit a region upstream from the scoring plane before reaching it. Therefore, the following classifications have been used in BEAMDP for the sub-source data processing ${ }^{2}$ for the particle origin and LATCH bit setting:

(1) For charged particles, the origin of the particle is considered to be the nearest (non-air) component that it has been to before it hits the scoring plane.

(2) The origin of a photon is considered to be the component in which it is created or scattered in the nearest component.

The reason for this classification is that for a charged particle, due to multiple scattering, its energy and direction are generally determined once it leaves the last non-air component. A photon may go through many regions before reaching the scoring plane, its energy is fixed when it is created or scattered.

It is required that each physical component in the treatment head has an associated LATCH bit during the full Monte Carlo simulation, and it is necessary to use inherited LATCH bit setting. There are several options for LATCH settings in the BEAMnrc code. In order to obtain the beam data necessary for beam modeling, the COMPREHENSIVE LATCH bit setting should be used in which the primary's LATCH values are inherited by the secondary particles to record where a particle has been for charged particles or has interacted for photons. Bit 0 tells if a bremsstrahlung photon is involved in the particle history.

\subsection{ABOUT MULTIPLE-SOURCE MODELS}




\subsubsection{Method of beam model design}

The methodology employed in beam model design is depicted in the flowchart in Figure 3.1. The "beam representation" is a concise mathematical description of the simulated phase-space data. "Beam reconstruction" involves reconstruction of phasespace data from the beam representation. The beam representation together with the method of reconstruction constitute the "beam model". First, BEAMnrc ${ }^{32}$ is

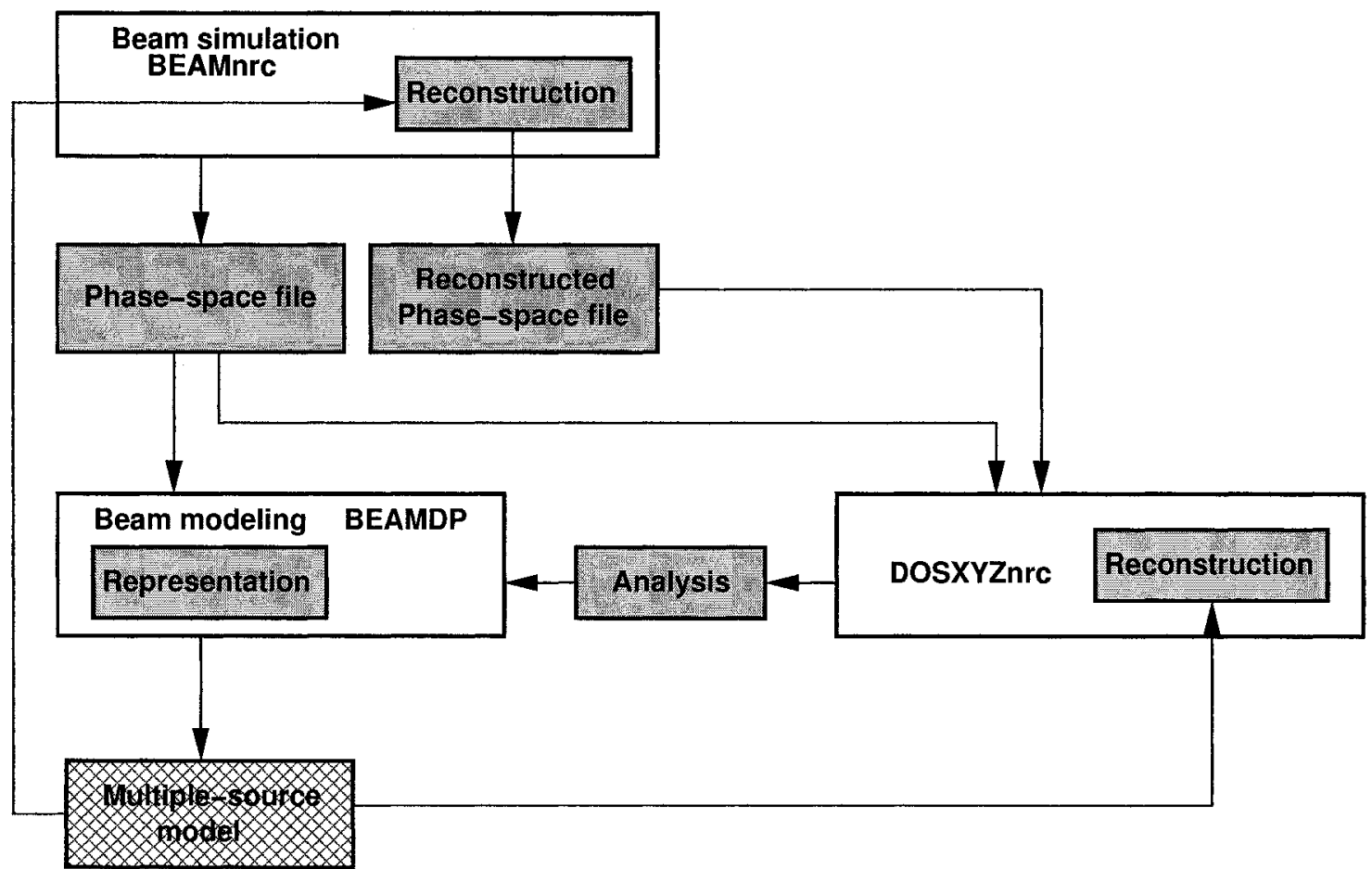

Figure 3.1: The methodology employed in the design and evaluation of a beam model.

used to calculate the raw beam and create the phase-space file at the scoring plane located at the end of the last component of the treatment head at SSD=100 $\mathrm{cm}$ (the top of the water phantom). The phase-space file contains all of the particles in the

\subsection{ABOUT MULTIPLE-SOURCE MODELS}


simulated beam that reached the scoring plane.

Details of the beam character are studied carefully with the emphasis on the types of particles in the beam, their intensities, dependence of the spectral distribution on the location, direction of the particles in the beam and mean energy distribution. After a beam model is specified and the parameters of the beam representation for a given simulated beam are determined, BEAMDP calculates the "beam representation" data: the relative number of particles from each accelerator component, planar fluence (relative numbers of particles per spatial bin), energy spectrum (relative numbers of particles per energy bin) for each of the energy regions and angular distribution of charged particles for correction of multiple-scattering in air.

Reconstruction of the particle phase-space from the beam representation is done with BEAMDP MORTRAN replacement macros in the BEAMnrc ${ }^{32}$ or DOSXYZnrc ${ }^{36}$ simulation codes. The reconstructed beams are analyzed and compared with the original phase-space data as a check on the beam modeling algorithm. The reconstructed beams can also be used by DOSXYZnrc for calculation of 3-D dose distributions in a rectilinear voxel phantom. The results can then be compared with the calculations using the original phase-space data. The number of sub-sources can be gradually reduced while maintaining the accuracy of the dose distribution calculation. It is an iterative process to optimize the number of sub-sources and to validate a model.

\subsection{Types of source models}

This section describes types of source models. Each commonly used component of clinical linear accelerator has a simplified 2-dimensional type of sub-source in the

\subsection{TYPES OF SOURCE MODELS}


plane perpendicular to z-axis which has the same dimensions along the $\mathrm{x}$ and $\mathrm{y}$ axes as the modeled component. The distance of the sub-source from the scoring plane is calculated from the mid-point of the component thickness.

There are a variety of sub-sources with respect to the components in an accelerator. They are classified as rings, cones and point sources, applicators, collimators, rectangular plane sources and circular plane sources.

\subsubsection{Ring or point sub-sources}

Primary collimators, which are usually ring or cone shaped, are modeled as a ring with zero height. The radial dimension of the sub-source is the same as that of the actual ring or cone at the mid-point of the ring/cone thickness. The distance from the sub-source to the scoring plane is calculated from the mid-point of the collimator thickness to the scoring plane. The particles are considered to be from the surface non-uniformly, with more coming from the edges of the opening because if the sampled particle falls inside the opening, then it is forced to be on the edges. When the radius of this sub-source is set to zero the sub-source becomes a point source.

The point source corresponds to the "direct" particles, particles coming directly from the exit window or bremsstrahlung target, and traversing components such as scattering foils or flattening filters, monitor chamber or mirror, without hitting any of the beam defining components such as collimators or applicators or scattering from the flattening filter. Source to surface distance (SSD) for a virtual point source is evaluated using a 'pin-hole' method explained in Section 3.5 (Ma et al. ${ }^{2}$ ).

\subsection{TYPES OF SOURCE MODELS}




\subsubsection{Applicator sub-sources}

Applicators are modeled as surfaces (square or rectangular rings) on the (x,y) plane with zero thickness. The dimensions of the applicator opening should be exactly the same as that of the applicator being modeled. It is not necessary, however, that the applicator model for charged particles have the same outer dimensions as those of the applicator, if the applicator's outer edges are shadowed by lower applicators (applicators closer to the scoring plane). The outer dimensions can be considered to be equal to the inner opening dimensions plus a 0.5 to $2.0 \mathrm{~cm}$ margin. For bremsstrahlung photons, the outer dimensions of the applicator should correspond to the area "exposed" to the electron beam, but in most cases, the actual outer dimensions can be used for the photon sources. The distance from the sub-source to the scoring plane is calculated from the mid-point of the applicator thickness to the scoring plane.

The particles are considered to be non-uniform on the surface, with more coming from the edges of the opening. That is because, if the sub-source has a central air region and the particle is sampled to fall inside the opening, then the particle is forced to be on the edges around the opening. This 'edge enhancement' is based on the observation that more particles come from the inner edges around the opening than from peripheral areas.

\subsubsection{Collimator sub-sources}

Collimator jaws are modeled as parallel-bars with zero height. The X and Y dimensions of the sub-source are the same as those of the actual collimator at it's mid-point thickness. The distance from the sub-source to the scoring plane is calculated from the mid-point of the collimator thickness to the scoring plane.

\subsection{TYPES OF SOURCE MODELS}


The orientation of the collimator bars can be either along the $\mathrm{x}$ or $\mathrm{y}$ axis. The particles are considered to be from the surface non-uniformly, with more particles coming from the edges of the opening (edge enhancement). The distance from the sub-source to the scoring plane is calculated from the mid-point of the collimator thickness to the scoring plane.

\subsubsection{Planar sub-sources}

Scattering foils, flattening filters, mirrors and monitor ionization chambers are modeled as either rectangular or circular planar sources. Planar sub-sources are mainly used for bremsstrahlung photons as they are created directly in these components and their origins are well-defined. For charged particles planar sub-sources should generally be replaced by a virtual point source.

The dimensions of the sub-source are the same as the actual dimensions at the mid-point of component thickness, for photon beam, or the area actually exposed to the electron beam, with zero thickness.

Particles are sampled uniformly on the source surface. The distance from the sub-source to the phantom surface can be calculated from the mid-point of the component thickness to the phantom surface.

\subsection{Planar fluence}

Each sub-source has its own particle planar fluence distribution, which is scored in spatial bins on the scoring plane. There are three field types for the planar fluence distribution (Figure 3.2):

\subsection{PLANAR FLUENCE}


1. a circular field with annular bins

2. a square field with square rings

3. a rectangular field with rectangular regions of equal area

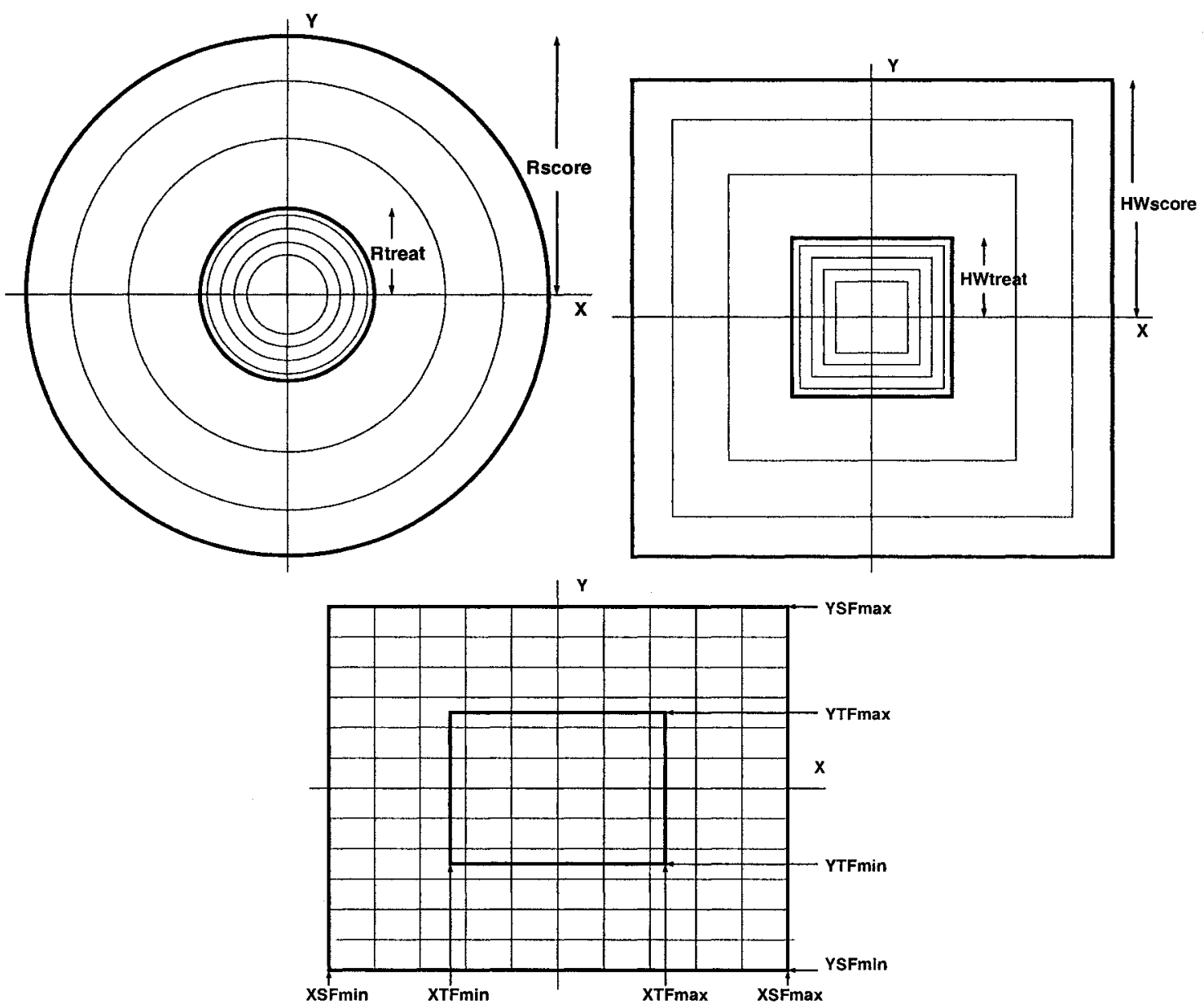

Figure 3.2: Schematic diagram for the circular, square and rectangular field types

The circular planar fluence scoring field is centered on the z-axis. It has $N_{t r}$ annular bins of equal area inside the region of the treatment field and $N_{s c o}$ annular bins of equal area outside the region of the treatment field. Equal bin area ensures less statistical fluctuation of the planar fluence from bin to bin for each region. Circu- 
lar fields are good for beams confined by circular linac components such as those scattered by scattering foils, monitoring chamber, mirror, and confined by ring- or cone-collimators. Fields formed by rectangular linac components such as jaws and applicators are not suitable for this field type.

The square planar fluence scoring field is also centered on the z-axis. The field is defined by its half-width (x-/y-directions), and divided into $N_{t r}$ square rings of equal area inside the region of the treatment field and $N_{s c o}$ square rings of equal area outside the region of the treatment field. In the old BEAMDP, the treatment field size was used to define the inside versus outside beam regions for spectra. For the new BEAMDP, the treatment field size for the planar fluence is independent of regions for the spectra.

The rectangular planar fluence scoring field allows the user to set-up asymmetric and/or off-axis fields. The rectangular field is divided into $N_{b i n} \times N_{b i n}$ equal rectangular areas to record the planar fluence, where $N_{b i n}$ is the number of bins for the planar fluence distribution. The user should specify the $\mathrm{x}$ and $\mathrm{y}$ dimensions of the scoring field.

The bins for the circular and square fields inside and outside the treatment field are not necessarily of equal size. The new BEAMDP code has the possibility of dividing the whole scoring field into two regions and specifying the number of equal area bins for each region individually. The treatment field region, which is of more interest, should be declared between $20 \%$ to $30 \%$ larger in size than the actual treatment field, in order to get a good spatial resolution in the area where the planar fluence usually has a very steep change, i.e. the field edge (Figure 3.3). The scoring field outside the treatment field region is of less interest. In the old BEAMDP implementation, the size of bins were equal for the whole scoring field, and

\subsection{PLANAR FLUENCE}


the highest resolution used to be at the edge of the scoring area, which is almost of no interest. Figure 3.3 presents the differences in planar fluence distribution using these two ways of spatial binning. It is evident that much better resolution of fluence is obtained inside the treatment field using the method implemented in the new BEAMDP code. When one divides the whole scoring field into $N_{t r}$ equal radial bins

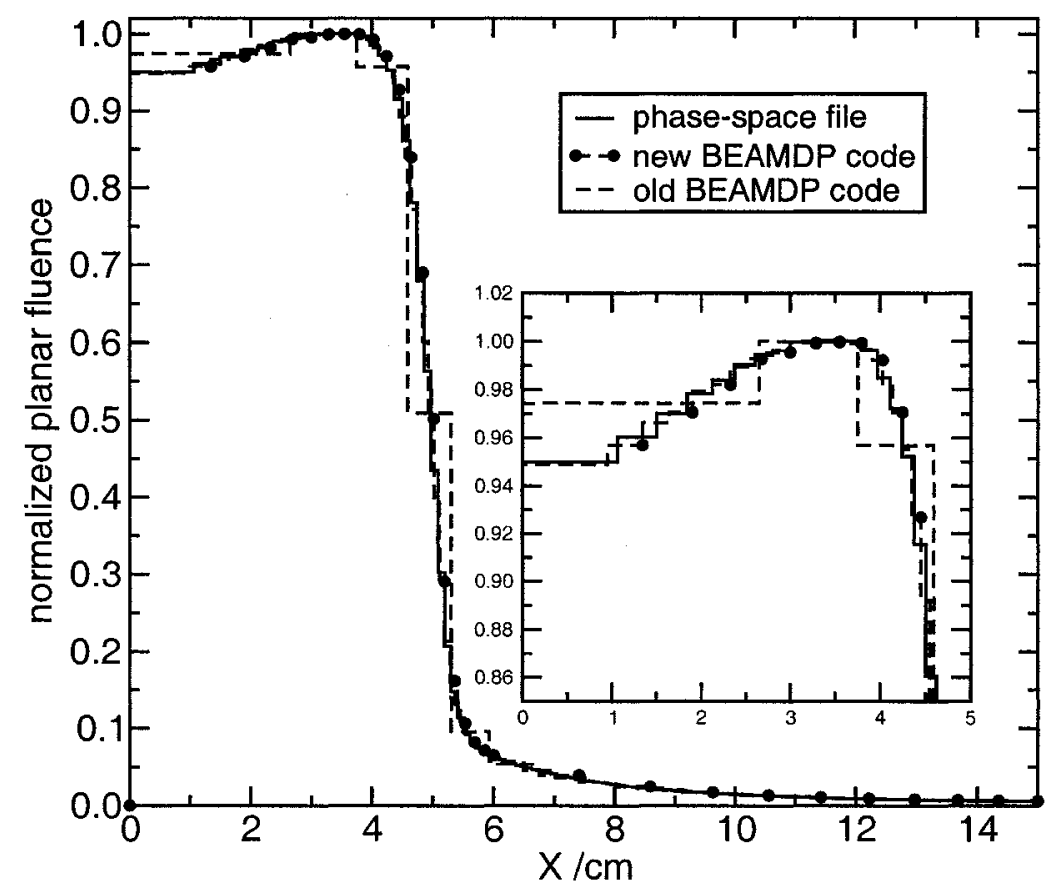

Figure 3.3: Comparison for the planar fluence scoring techniques between the two versions of BEAMDP code against the original phase-space file: (1) the planar fluence obtained using the new BEAMDP code with 60 planar fluence bins (40 bins for inside +20 bins for outside of the treatment field, (2) the planar fluence obtained using the old BEAMDP code and model with 64 planar fluence bins

inside the treatment field and $N_{s c o}$ equal bins outside the treatment field as shown on Figure 3.2, then the total number of bins is equal to $N=N_{t r}+N_{s c o}$. The outer radius of each bin inside the treatment field is given by

$$
r_{i}=\sqrt{\frac{i}{N_{t r}}} R_{t r}
$$


for

$$
i=1,2, . ., N_{t r}
$$

The radius of each bin outside the treatment field and inside the scoring field is given by

$$
r_{N_{t r}+i}=\sqrt{\frac{i}{N_{s c o}}\left(R_{s c o}^{2}-R_{t r}^{2}\right)+R_{t r}^{2}}
$$

for

$$
i=1,2, . ., N_{s c o}
$$

The same formulas can applied for square rings as in Figure 3.2 where $r_{t r}$ stands for the half-width of a treatment field and $r_{s c o}$ for the half-width of a scoring field. For example, if a treatment field size of $10 \times 10 \mathrm{~cm}^{2}$ is divided into 5 equal bins, and a scoring field size of $20 \times 20 \mathrm{~cm}^{2}$ is divided into 3 equal bins which excludes the treatment field, then the total number of bins is $N=N_{t r}+N_{s c o}=5+3=8$ and the outer dimensions are $r_{1}=2.23 \mathrm{~cm}, r_{2}=3.16 \mathrm{~cm}, r_{3}=3.87 \mathrm{~cm}, r_{4}=4.47 \mathrm{~cm}$, $r_{5}=5.00 \mathrm{~cm}, r_{6}=7.70 \mathrm{~cm}, r_{7}=8.66 \mathrm{~cm}, r_{8}=10.00 \mathrm{~cm}$. In this example the bins out of the treatment field are much bigger in size $\left(100 \mathrm{~cm}^{2} / \mathrm{bin}\right)$ than the bins inside the treatment field $\left(20 \mathrm{~cm}^{2} / \mathrm{bin}\right)$.

\subsection{Energy Spectra}

BEAMDP analyzes the BEAM phase-space data and generates energy distributions for each of the energy regions. The outer dimensions of the energy regions are given by the user and could be of any size equal or lower than $R_{\text {score }}$ and $H W_{\text {score }}$ for circular and square field types, respectively. The type of the energy regions is the same as the fluence field type, i.e. if the fluence is square rings then the energy regions are forced to be too. The outer dimension of the last energy regions must comply with the size

\subsection{ENERGY SPECTRA}


of the scoring field. The minimum number of energy regions is two, for the region inside the treatment field and the region outside the treatment field. The maximum number of energy regions allowed is 200 but could be easily changed by modifying a single macro in the source code (variable name $\$ \mathrm{NB}$ ).

This feature of multi-region spectral distributions was implemented as part of this project. It is based on the fact that the mean particle energy could vary significantly not only around the treatment edge but also inside or outside the treatment field. Figure 3.4 shows the good agreement for the mean energy of photons along $\mathrm{x}$-axis for the reconstructed $25 \mathrm{MV}$ photon beam, $10 \times 10 \mathrm{~cm}^{2}$ field using the new BEAMDP code for ten energy regions inside the scoring field against the original phase-space file. The old BEAMDP code gives one distribution with fixed mean energy inside and one outside the treatment field with another mean energy. Figure 3.5 presents the energy spectra obtained from the original phase-space file and a multiplesource model. Both models reproduce the fine details of the energy spectrum for the $18 \mathrm{MeV}$ electron beam with $10 \times 10 \mathrm{~cm}^{2}$ field size simulated with the monoenergetic incident electron beam source. The three peaks in the spectrum are caused by the complex (layered) geometric structure of the secondary scattering foil in the Elekta SL25 linear accelerator. The difference in the spectra between the new BEAMDP code and the old BEAMDP code is because of the difference in the energy regions for both cases. The model for the new BEAMDP code is for 10 energy regions, and the spectrum is obtained in the region $0-2.5 \mathrm{~cm}$ half-width from the z-axis, while the old BEAMDP model spectrum is inside the entire treatment field ( $5 \mathrm{~cm}$ half-width). Generally, having the ability to create more energy spectra gives greater possibilities for fine tuning of the beam models (Figures 3.5 and 3.6).

3.4. ENERGY SPECTRA 


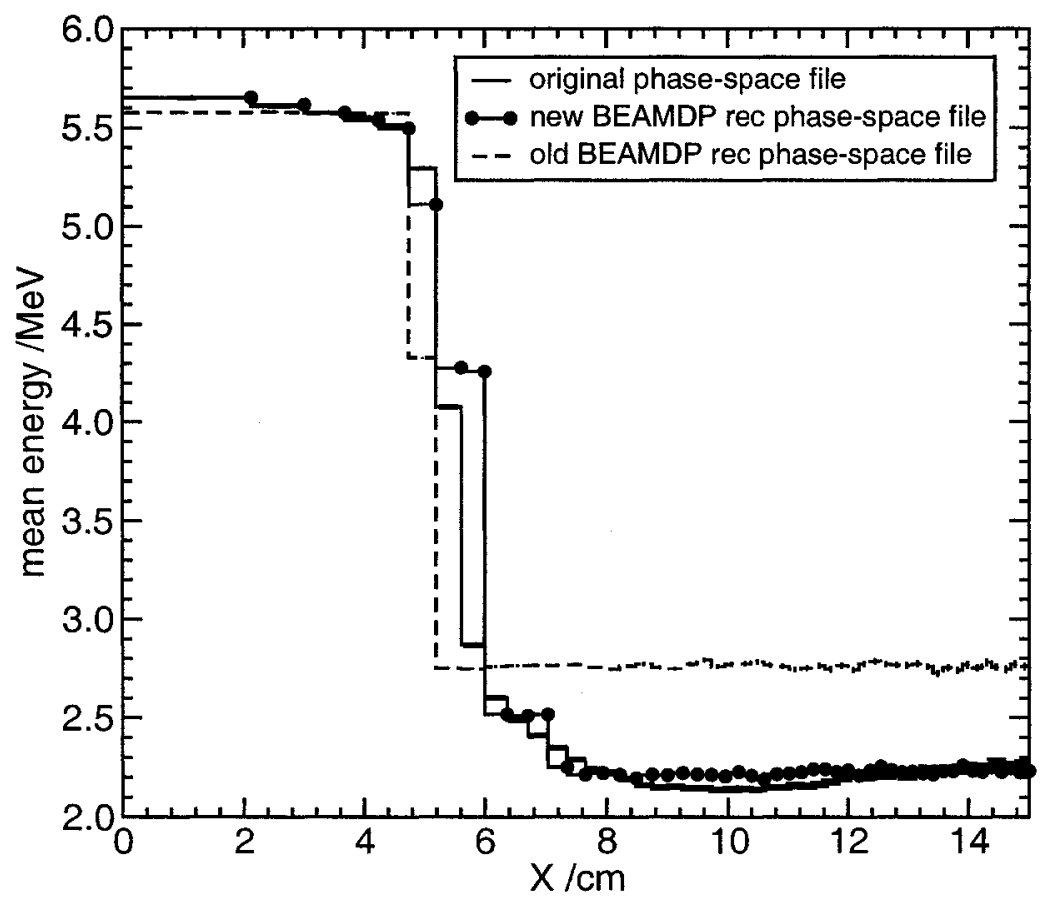

Figure 3.4: Comparison for the mean energy distribution of entire photons between the two versions of BEAMDP code against the original phase-space file for $25 \mathrm{MV}$ photon beam, with a $10 \times 10 \mathrm{~cm}^{2}$ field. Reconstructed phase-space files were obtained from the model with one point sub-source of photons. (1) reconstructed phase-space file obtained using the new BEAMDP code with 10 square energy regions with the half-width size of $2.0,3.0,4.0,4.5,5.0,5.5,6.0,6.5,7.0,8.0,10.0$ and $15.0 \mathrm{~cm}$. Mean energy distribution is llat inside each of energy regions. (2) reconstructed phase-space file obtained using the old BEAMDP code with energy spectra inside and outside the treatment field. The mean energy distribution is flat inside and outside the treatment field. 


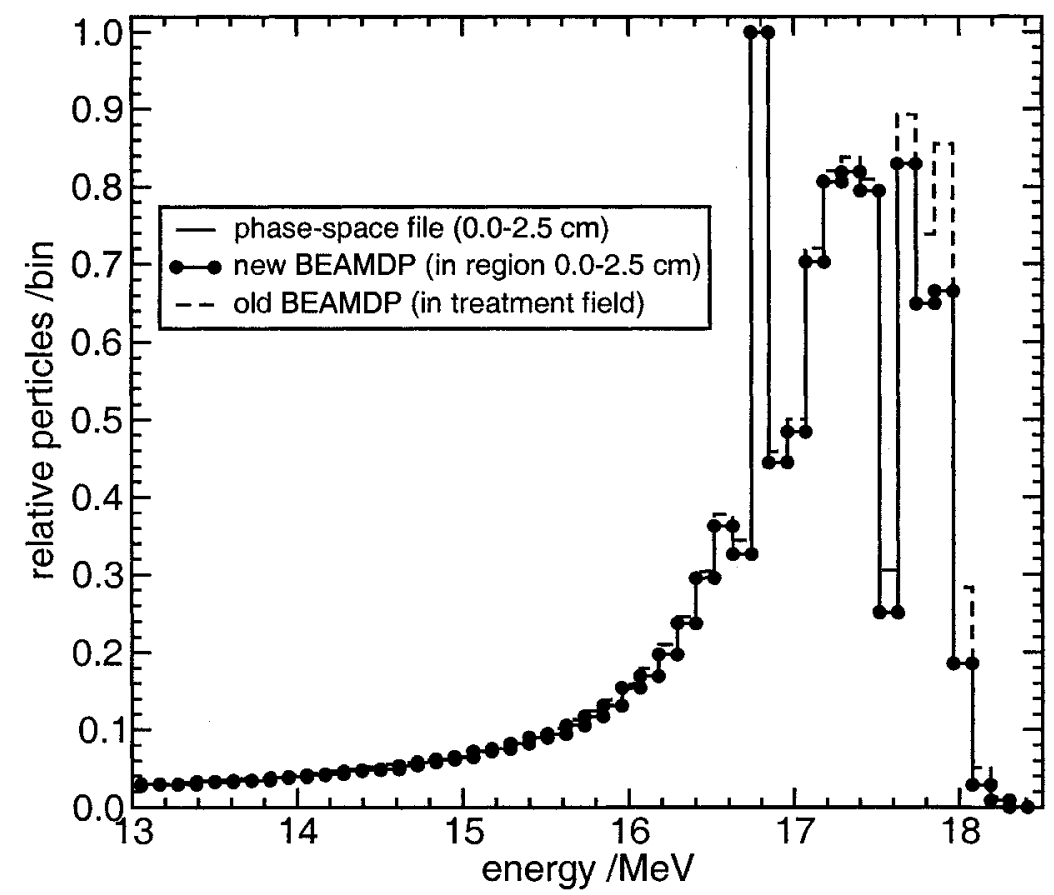

Figure 3.5: Comparison of the electron energy spectra for the two versions of the BEAMDP code against the original phase-space file for the $18 \mathrm{MeV}$ electron beam, $10 \times 10 \mathrm{~cm}^{2}$ field size simulated with the monoenergetic incident electron beam source. The electron energy spectrum for the phase-space file is inside the square region 0 $2.5 \mathrm{~cm}$ half-width. The electron energy spectrum obtained using the new BEAMDP code and a model with 10 energy regions, is for the same region, while the spectrum obtained using the old BEAMDP code and model with energy spectra inside the treatment field ( $5 \mathrm{~cm}$ half-width). The same number of energy bins is used for both models. All curves are normalized to the maximum value at $16.8 \mathrm{MeV}$. 


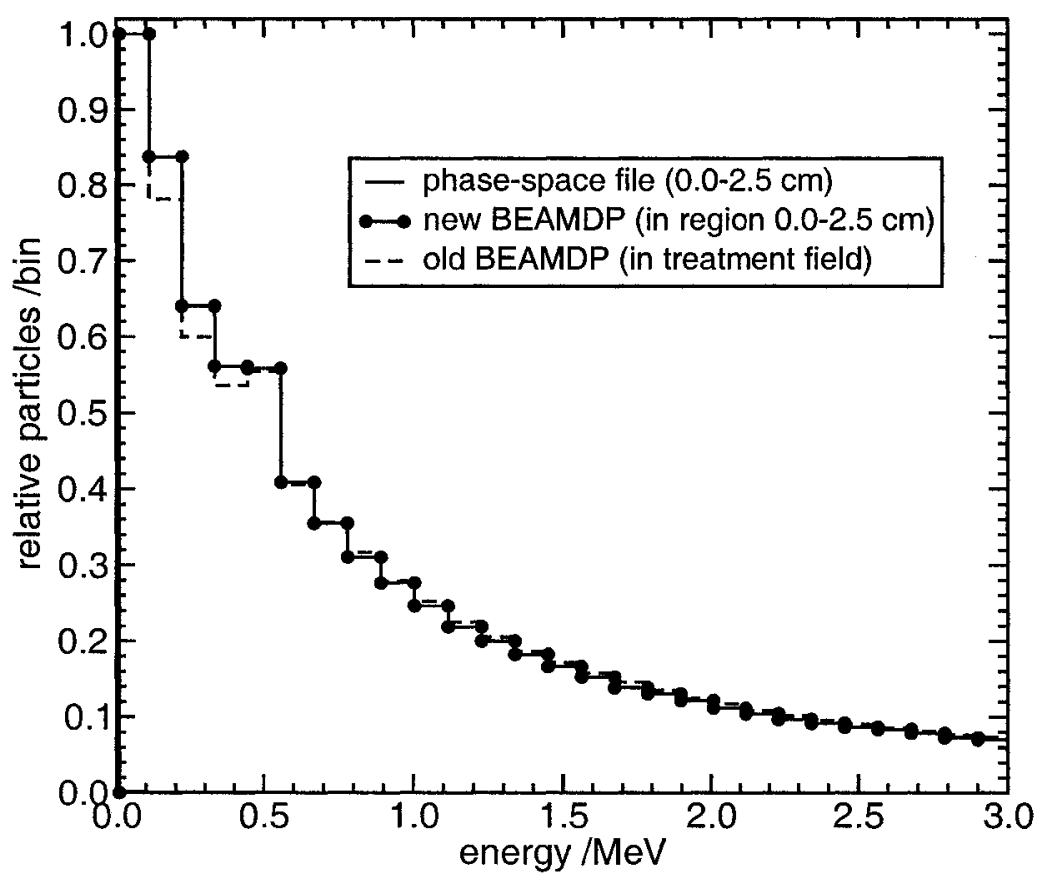

Figure 3.6: Comparison of the photon energy spectra for the two versions of the BEAMDP code against the original phase-space file for the $18 \mathrm{MeV}$ electron beam, $10 \times 10 \mathrm{~cm}^{2}$ field size simulated with the monoenergetic incident electron beam source. The photon energy spectrum for the phase-space file is inside the square region 0 $2.5 \mathrm{~cm}$ half-width. The photon energy spectrum obtained using the new BEAMDP code and a model with 10 energy regions, is for the same region, while the spectrum obtained using the old BEAMDP code and model with energy spectra inside the treatment field ( $5 \mathrm{~cm}$ half-width). The same number of energy bins is used for both models. All curves are normalized to the maximum value at $0.1 \mathrm{MeV}$. 


\subsection{Pin-hole method}

When the multiple-source model contains a point source for 'direct' particles, one needs a suitable algorithm to calculate the virtual distance of the source to the surface of the water phantom $\left(S S D_{\text {vir }}\right)$ where the scoring plane was placed. Several methods have been tested as described by Ma et al. ${ }^{2}$ A method similar to the 'pin-hole' technique, which uses an annular aperture instead of pin-holes, was selected as the most suitable and accurate one. In this method, the phase-space particles which

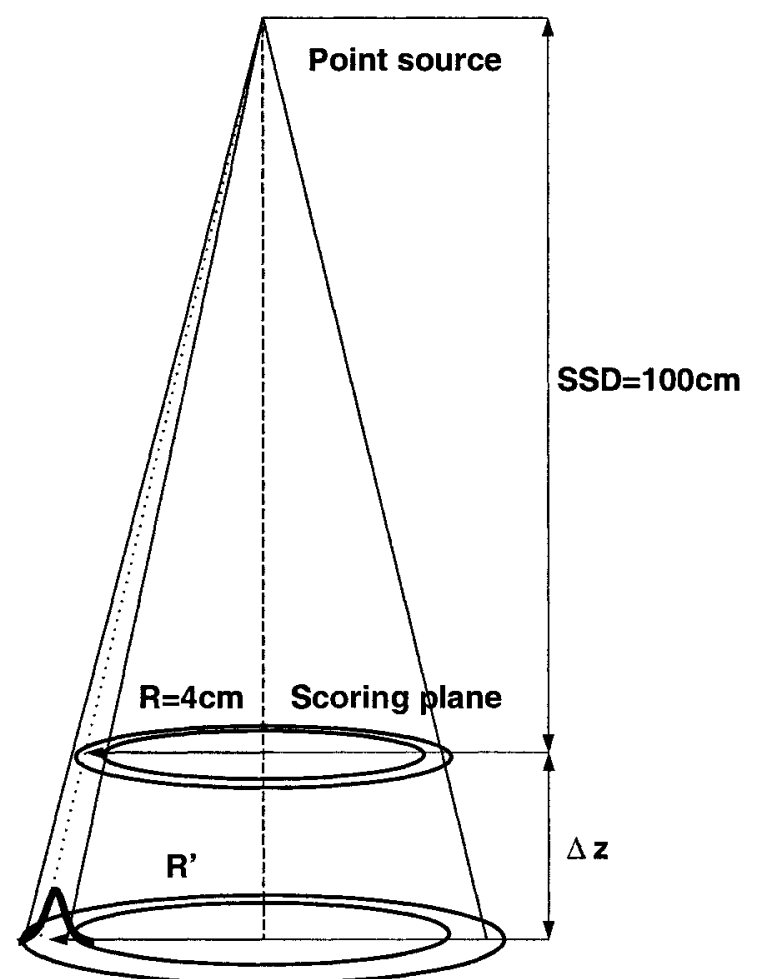

Figure 3.7: Pinhole method to calculate the virtual SSD of particle point source in $\mathrm{BEAMDP}$ code. $\mathrm{SSD}=100 \mathrm{~cm}$, radius $\mathrm{R}=4 \mathrm{~cm}$ so that annular aperture lies inside the $10 \times 10 \mathrm{~cm}^{2}$ field size. This picture is not to scale.

are crossing through a thin annular aperture with a radius $\mathrm{R}$ form an image at a

\subsection{PIN-HOLE METHOD}


distance $\Delta \mathrm{z}$ in vacuum beyond the scoring plane (Figure 3.7). The radius $\mathrm{R}$ of the annular aperture has to fit inside the treatment field. The aperture image has a peak at radius $\mathrm{R}^{\prime}$. The distance from the scoring plane to the virtual focal spot, $S S D_{v i r}$ can be calculated from

$$
S S D_{\mathrm{vir}}=\frac{R}{R^{\prime}-R} \Delta z
$$

For example, the $S S D_{\text {vir }}$ value obtained using this method, for the $18 \mathrm{MeV}$ electron beam, $10 \times 10 \mathrm{~cm}^{2}$ field size, is estimated to be $97.77 \mathrm{~cm}$ for the direct electron subsource and $98.75 \mathrm{~cm}$ for the direct photon sub-source. For the $25 \mathrm{MV}$ photon beam, $10 \times 10 \mathrm{~cm}^{2}$ field size, the values obtained are $99.75 \mathrm{~cm}$ and $75.38 \mathrm{~cm}$ for the direct photons and electrons, respectively.

The radius $R=4 \mathrm{~cm}$ has been used. The dose distribution calculated using these values are presented in the following chapter.

If the result for $S S D_{\text {vir }}$ of a particular point sub-source is a negative number, then particles do not back project to any point above the scoring plane. In that case the sub-source cannot be modeled as a point source. If the relative intensity of that source is very low, there are not enough particles to use this method. The source can either be ignored or modeled as another source type.

The 'pin-hole' method described above has been implemented in the BEAMDP code to calculate the virtual source-to-surface-distance (SSD) for all point sources. ${ }^{2}$

\subsection{Beam reconstruction}

The full phase-space data can be re-constructed from a simplified sub-source model using the relative intensity of sources and their energy and planar fluence distribu-

\subsection{BEAM RECONSTRUCTION}


tions, while the particle angular distribution and their correlations with energy and position are determined from the geometry details of the sub-sources and by the sampling methods. The reconstruction of phase-space data is done in five steps:

1. Relative source intensity sampling

The first step in the beam re-construction is to sample particles according to relative intensities of the sub-sources in a model. Relative intensity is a ratio of the number of particles coming from a particular source and the total number of particles. Since a sub-source only radiates one type of particle (photon, electron or positron), sampling of a sub-source will determine the particle's charge.

\section{Energy sampling}

Each sub-source has its own energy spectrum within each energy region. The number of energy regions and energy bins used for the spectrum is specified by the user. It has a minimum energy which must be equal to or smaller than the lowest energy in the beam (usually PCUT), and a maximum energy which must be equal to or higher than the highest particle energy in the beam.

3. Particle position sampling

Each sub-source has its own associated planar fluence distribution from which the particle's position on the phase-space plane is sampled. The type and number of bins used for the planar fluence is specified by the user.

4. Incident angle sampling

To determine the particle's incident angle, the geometry of the sub-source is used to sample a point on it defined by the sub-sources dimensions. The sampling is based on a probability distribution

$$
P_{\text {scat }}=\frac{1}{1+\left(\frac{r}{R_{\text {scat }}}\right)}
$$

\subsection{BEAM RECONSTRUCTION}


where $r$ is the distance from the point to the already chosen particle position (step 3) on the same plane, and $R_{\text {scat }}$ is the scatter radius which can be calculated from $R_{\text {scat }}=\bar{\theta} Z_{\text {min }}$ where $\bar{\theta}$ is the electron mean scattering angle from the model and $Z_{m i n}$ is the distance from the mid-point of the thickness of the component to the scoring plane. If a sampled point falls into the opening of the component, it will be moved to the edge of the opening. The angle of the particle is then determined by the position of the particle on the phantom surface and the sampled point at the sub-source.

\section{Air scattering}

This final step in the beam reconstruction is to correct for the air scattering of charged particles. By analyzing the simulated phase-space file, an angular spread (angle between the particle direction and the z-axis) is scored for the direct electrons within a circle of $1 \mathrm{~cm}$ radius. The angular spread is considered to be a good approximation for a pencil beam of electrons of the same energies going through an air slab of thickness equal to the $S S D_{\text {direct }}$ of the direct electrons. This angular distribution is used for sampling the angular fluctuation of charged particles around their directions. If a sub-source is at the smaller distance $S S D_{s u b}$, the sampled scattering angle is scaled down by the factor $S S D_{\text {sub }} / S S D_{\text {direct }}$.

\subsection{Phase-space reconstruction}

Source models are built from phase-space files. To reconstruct the phase-space data in a DOSXYZnrc calculation, the source option "Beam Characterization Model Incident From any Direction" must be used. However, to reconstruct the phase-space file from

\subsection{PHASE-SPACE RECONSTRUCTION}


the model, the BEAMnrc code must be used with the source option "Phase Space Reconstructed Using Beam Models".

Using these source options beam data are derived from a beam characterization model using the same sampling procedure described in the previous section. There is no limit to the number of particles in a phase-space reconstruction, so that basically statistics can be significantly improved for the reconstructed phase-space. However, the reconstructed phase-space may differ substantially from the original (simulated) one, depending on the design of the beam model and its accuracy. On the other hand, computation time for phase-space reconstruction is significantly shorter than computation time for a full treatment head simulation but is comparable to the time required to re-use the original phase-space file.

BEAMnrc and DOSXYZnrc calculation descriptions are given in Section 4.4.

\subsection{Model verification}

A good model gives a good match of depth-dose curves and dose-profiles calculated with the original phase-space file. Therefore, a model can be verified dosimetrically by comparing the dose distribution in a water phantom using calculations from the original phase-space file versus calculations from the multiple-source model, or, by using the original phase-space file versus a reconstructed phase-space file in calculations. Ideally, one wants a model with as few sub-sources as possible while maintaining accuracy in the dose distribution calculations. Calculation procedures are explained in Chapter 4 while examples and results are given and discussed in Chapter 6. 


\section{Chapter 4}

\section{Monte Carlo calculations}

In this chapter Monte Carlo calculations done for this project are addressed. Following the method of beam model design (Figure 3.1), BEAMnrc Monte Carlo simulations of the NRC Elekta SL25 treatment head were started to establish the foundations on which the multiple-source beam models were built. A collection of phase-space files was created for both photon and electron beams and for all combinations of supported energies and applicators (Table 2.1). As estimated by Faddegon et al. ${ }^{43}$ even for users with Monte Carlo simulation experience, it takes about two months of CPU time to generate a complete set of beam data for a single accelerator. This proved to be the most tedious and time consuming part of the project since much effort was put in trying to obtain the best agreement with experiment using different input parameters and repeatedly executing calculations, which in some cases, especially for the photon beams, took several days to complete. Multiple-source beam models could not be done without this step.

The next step, analysis and creation of beam models, for which purpose the existing BEAMDP program had to be modified, was also a time consuming task. 
Although the software modifications in BEAMDP code took only a few weeks, the testing and debugging of software was slow, with some problems that were not obvious for photon beams but critical for electron beams. Both versions of the BEAMDP software were used to create and analyze models. Software modifications brought some changes to the command line interface of the program, so both, old BEAMDP input files and new BEAMDP input files were maintained for each model. Using both versions of software was a good test for the accuracy of the modified BEAMDP code, and also a good reference to observe improvements in the results, as it was already seen in Chapter 3. For each energy, five different models were designed, ranging from the most complex one which contains all components, to the most simple one, which includes only one direct source for photons and one direct source for electrons. The BEAMnrc code and MORTRAN beam model replacement macros were used to reconstruct the phase-space files from the multiple-source beam models.

For model verification purposes, the DOSXYZnrc code was used for dose distribution calculations from the original phase-space files, multiple-source models and reconstructed phase-space files - in a rectangular voxel based phantom. Dosedistribution curves obtained from the models were compared against dose-distribution curves from the simulated phase-space file to validate the model.

\subsection{BEAMnrc calculations}

In the present calculations constant values of $A E=E C U T=0.7 \mathrm{MeV}$ and $A P=$ PCUT $=0.01 \mathrm{MeV}$ have been generally used for all component modules in the BEAMnrc simulations with the exception of scoring a phase-space file, usually at the last component just before the water phantom. When scoring the phase-space file,

\subsection{BEAMNRC CALCULATIONS}


to prevent backscatter radiation from the phantom, the cutoff energies of the next component must be set to some high value (higher than the maximum energy in a beam).

Since low-energy electron transport constitutes a major part of the total CPU time for the simulation, BEAMnrc's range rejection technique (with set ECUTRR) ${ }^{49}$ has been used in all simulations. For each medium in an accelerator, EGSnrc calculates a table of ranges to the electron transport cutoff energy (ECUT) as a function of electron energy. These ranges are calculated using restricted stopping powers and thus represent the longest possible ranges to the cutoff energy. When range rejection with a set $E C U T R R$ is chosen, range rejection is performed on a region by region basis. If the range to the cutoff energy $(E C U T R R)$ is less than the distance to the nearest region boundary, the history is terminated and energy is deposited in the current region. Range rejection introduces an approximation because, in terminating a charged particle's history and depositing all of its energy in the current region, it is assumed that any bremsstrahlung photons that would have been created by the particle do not leave the current region. One can minimize inaccuracy resulting from this approximation by using the variable $E S A V E$ which defines the maximum charged particle energy (in $\mathrm{MeV}$ ) at which range rejection is considered. Electrons having an energy larger than $E S A V E$ are not considered for range rejection since they may produce bremsstrahlung photons which might escape the region. Sheikh-Bagheri ${ }^{50}$ evaluated that a value of $E S A V E=2 \mathrm{MeV}$ provides a three-fold increase in the speed of the calculations and ignores only $0.1 \%$ of the photons reaching scoring plane at an SSD of $100 \mathrm{~cm}$. These ignored photons are bremsstrahlung photons produced in a linac geometry at an energy below $E S A V E$.

Anywhere but outside the target (modeled with CM SLABS where one has

\subsection{BEAMNRC CALCULATIONS}


the ability to assign another value) if the electron's total energy falls below $2.0 \mathrm{MeV}$ (ESAVE) and its range does not allow it to escape the geometric region it is already traveling in, then it is discarded and its energy scored locally. For the slab target and copper base, ESAVE values are set to 0.6 and $1.00 \mathrm{MeV}$, respectively, to provide higher accuracy for bremsstrahlung production.

For the incident electron beam the source was a circular parallel beam in which the incident particles have:

1. identical Gaussian distributions in the $\mathrm{X}$ and $\mathrm{Y}$ directions

2. angular distribution

3. energy distribution

described as an ISOURC=19: "Parallel Circular Beam with 2-D Gaussian X-Y Distribution" in the BEAMnrc manual.

The full Monte Carlo simulation of a treatment head (Figure 2.1) produces a phase-space file which includes the position, charge, energy, weight and LATCH of every particle (Equation 1.3, page 14), while the reconstructed phase-space file from a model includes only position, charge and energy. The weight and LATCH bits are not reconstructed from the model and they are set to 1 and 0 , respectively:

$$
\left\{H_{i} \mid i=1,2,3, . . N\right\} \mathrm{with}_{i}=\left(x_{i}, y_{i}, u_{i}, v_{i}, q_{i}, E_{i}, 1,0\right)
$$

\subsubsection{Photon beams}

Simulating photon beams from an Elekta SL25 takes much longer than for electron beams since larger number of particle histories is required in order to achieve the

\subsection{BEAMNRC CALCULATIONS}


desired level of precision. Photon beam simulations are run in parallel on 10 Pentium 2.4 GHz processors. Table 4.1 shows how many electron histories are simulated for each photon beam energy and field size. It is evident that lower number of histories is required for higher energies to get the same statistics in dose calculation.

Table 4.1: Initial number of histories (in millions) and size of phase-space files (PSF) in MB for various photon beam energies and field sizes to create phase-space data at $\mathrm{SSD}=100 \mathrm{~cm}$. The phase-space data are scored in $\mathrm{x}-\mathrm{y}$ plane of $30 \mathrm{~cm}$ radius centered about the beam axis. Phase-space information for one particle is 28 bytes.

\begin{tabular}{lccc}
\hline \hline Energy & Field size & Histories $\left(10^{6}\right)$ & PSF size (MB) \\
\hline $6 \mathrm{MV}$ & $10 \times 10 \mathrm{~cm}^{2}$ & 40 & 625 \\
& $40 \times 40 \mathrm{~cm}^{2}$ & 10 & 2829 \\
$10 \mathrm{MV}$ & $10 \times 10 \mathrm{~cm}^{2}$ & 20 & 1742 \\
& $40 \times 40 \mathrm{~cm}^{2}$ & 10 & 6703 \\
$25 \mathrm{MV}$ & $10 \times 10 \mathrm{~cm}^{2}$ & 20 & 609 \\
& $40 \times 40 \mathrm{~cm}^{2}$ & 10 & 4493 \\
\hline \hline
\end{tabular}

The directional bremsstrahlung splitting (DBS) variance reduction technique has been used in the simulation of clinical photon beams with splitting field radius of $30 \mathrm{~cm}$, and brem splitting number of 400 for the $6 \mathrm{MV}$ photon beam. For the $25 \mathrm{MV}$ photon beam, a splitting field radius of $21 \mathrm{~cm}$ and brem splitting number of 500 were used. It was an oversight using a $21 \mathrm{~cm}$ splitting field radius since it does not cover the corners of the $40 \times 40 \mathrm{~cm}^{2}$ field. For the $6 \mathrm{MV}$ beam, $10 \times 10 \mathrm{~cm}^{2}$ field size, with 40,000,000 initial electrons, the phase-space file contains a total of 22,335,137 particles of which $22,204,803$ are photons and 130,334 are electrons. That is 560 photon entries per 1000 electrons incident on the target. These entries have weights much less

\subsection{BEAMNRC CALCULATIONS}




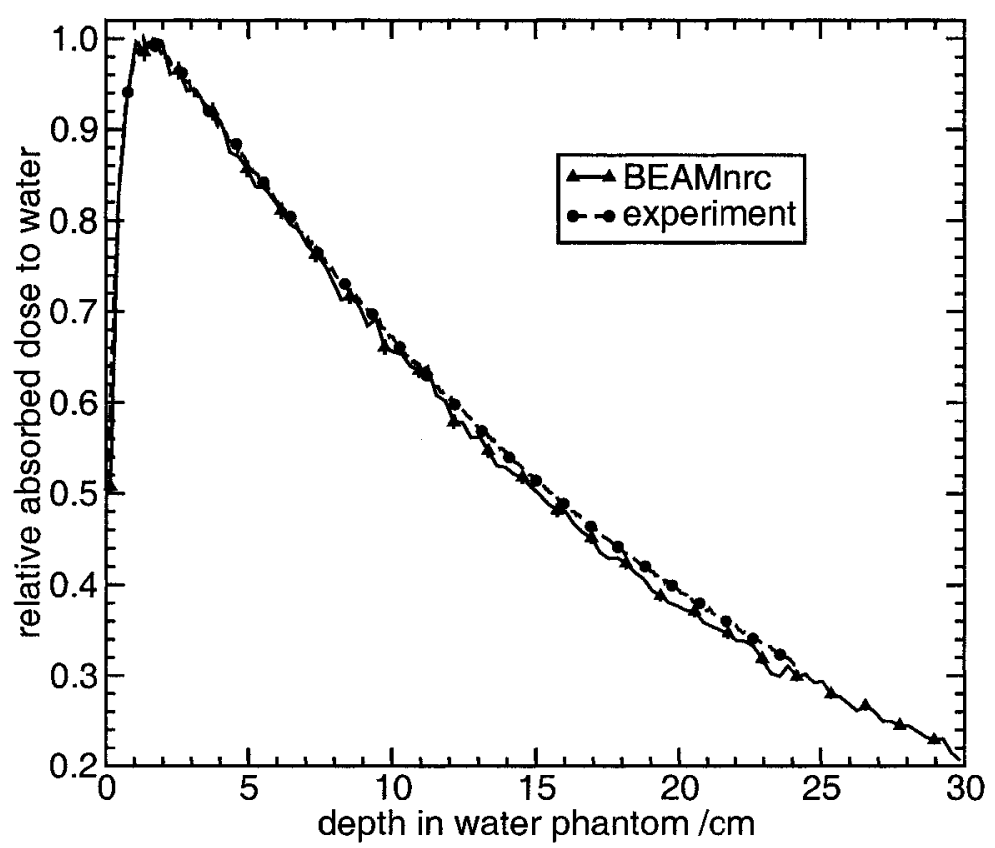

Figure 4.1: Comparison of measured and calculated central-axis depth-dose curves for the NRC Elekta SL25 $6 \mathrm{MV}$ photon beam. Uncertainties of experimental results are within 1\%. Depth of the phantom is $30 \mathrm{~cm}$ (Section 4.3), scoring region is $41 \mathrm{x} 1$ $\mathrm{cm}$ along $\mathrm{x}$-axis. The field size is $10 \times 10 \mathrm{~cm}^{2}$ at an SSD of $100 \mathrm{~cm}$.

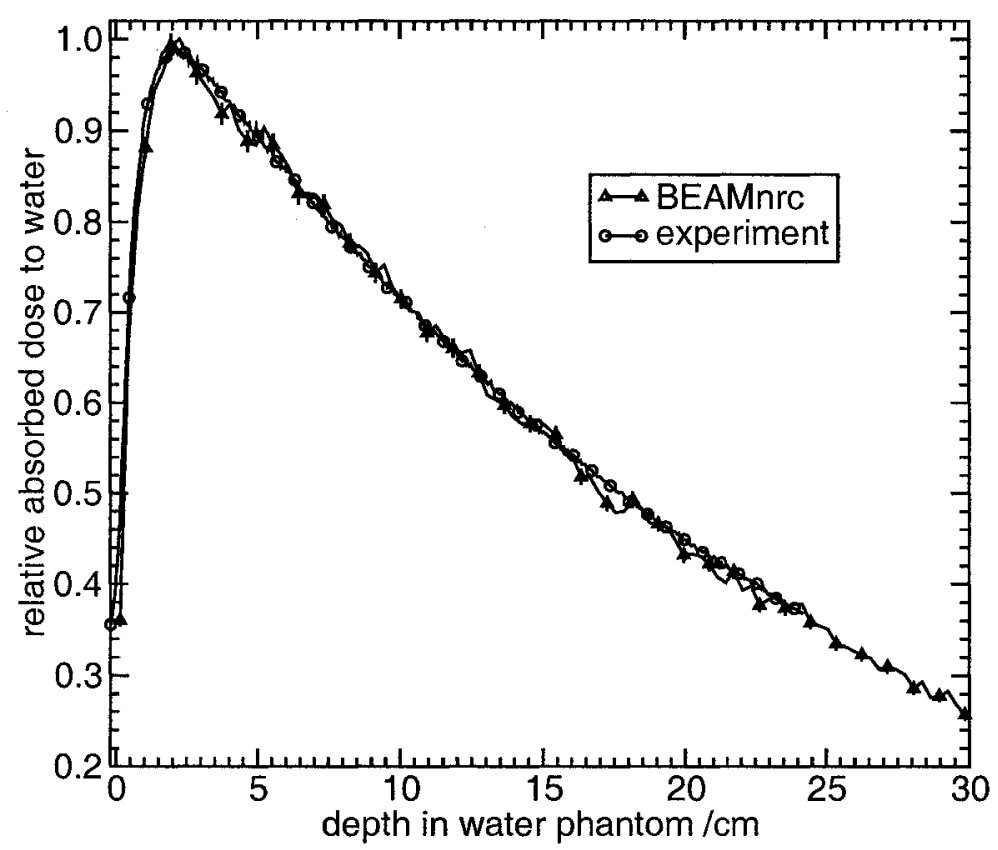

Figure 4.2: Comparison of measured and calculated central-axis depth-dose curves for the NRC Elekta SL25 $10 \mathrm{MV}$ photon beam. Depth of the phantom is $30 \mathrm{~cm}$ (Section 4.3), scoring region is $41 \times 1 \mathrm{~cm}$ along x-axis. The field size is $10 \times 10 \mathrm{~cm}^{2}$ at an SSD of $100 \mathrm{~cm}$.

\subsection{BEAMNRC CALCULATIONS}


than 1 inside the region of interest and correspond to only 1.38 physical photons per 1000 incident electrons. The $6 \mathrm{MV}$ photon beam phase-space file corresponds to $0.662 \mathrm{~GB}$ of data. For the $25 \mathrm{MV}$ beam with $20,000,000$ initial electrons, the phasespace file contains a total of 21,751,049 particles of which $21,302,517$ are photons and 359,391 are electrons. There are 1088 photon entries per 1000 electrons incident on the target or 2.72 physical photons per 1000 incident electrons. The $25 \mathrm{MV}$ photon beam phase-space file corresponds to $0.609 \mathrm{~GB}$ of data. With these phase-space files the statistics achieved for depth-dose calculation was better than $1 \%$. The depth-dose simulation results for the $6 \mathrm{MV}$ and $10 \mathrm{MV}$ photon beams, based on the input files and measurements obtained from NRC, are presented in Figures 4.1 and 4.2.

The dose-profile simulation result for the $25 \mathrm{MV}$ photon beams, based on the input files and measurements obtained from NRC, are presented in Figure 4.3. 


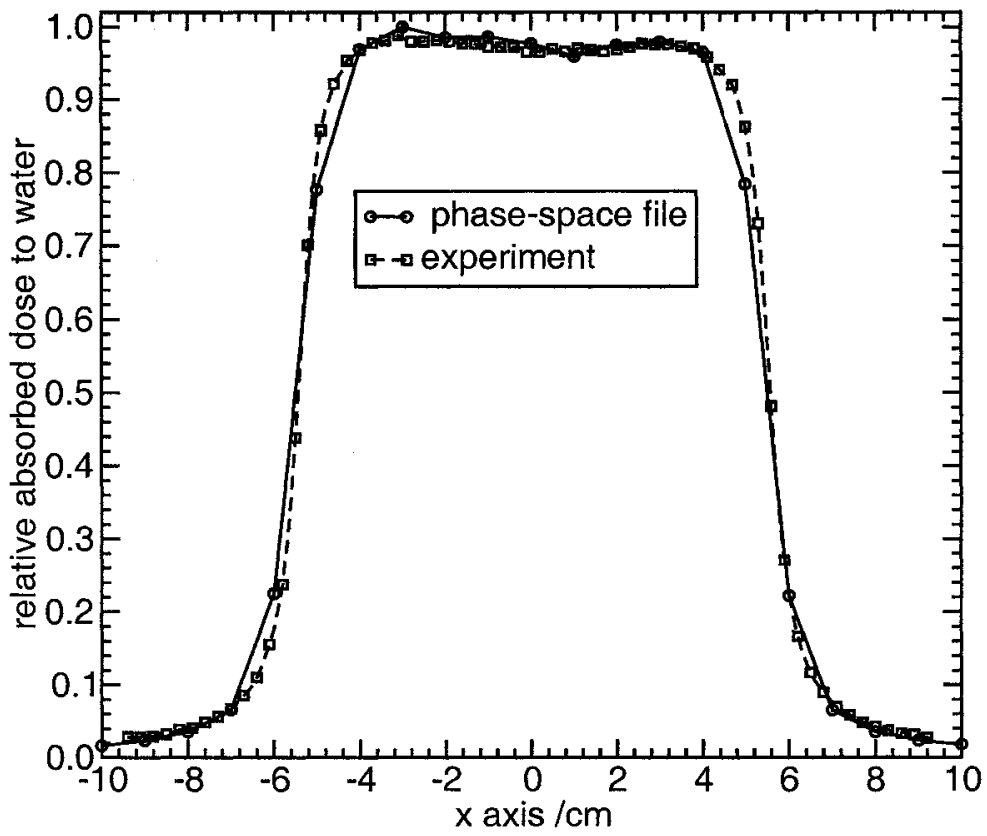

Figure 4.3: Comparison of measured and calculated dose-profiles for the NRC Elekta SL25 $25 \mathrm{MV}$ photon beam. Depth of the phantom is $30 \mathrm{~cm}$ (Section 4.3), scoring region is $41 \times 1 \mathrm{~cm}$ along $\mathrm{x}$-axis. The field size is $10 \times 10 \mathrm{~cm}^{2}$ at an SSD of $100 \mathrm{~cm}$. The curves were normalized to the value at $d_{\max }$. 


\subsubsection{Electron beams}

No parallel job processing or bremsstrahlung splitting were used for electron beam simulations. The number of particles stored in the phase space file depends on the

Table 4.2: Initial number of histories (in millions) and size of phase-space files (PSF) in MB for various electron beam energies and field sizes to create phase-space data at $\mathrm{SSD}=100 \mathrm{~cm}$. The phase-space data are scored in the $\mathrm{x}-\mathrm{y}$ plane for a radius of $30 \mathrm{~cm}$ centered about the beam axis. Phase-space information for one particle is 28 bytes.

\begin{tabular}{cccc}
\hline \hline Energy & Field size & Histories $\left(10^{6}\right)$ & PSF size (MB) \\
\hline $4 \mathrm{MeV}$ & $10 \times 10 \mathrm{~cm}^{2}$ & 80 & 155 \\
& $20 \times 20 \mathrm{~cm}^{2}$ & 60 & 240 \\
& $40 \times 40 \mathrm{~cm}^{2}$ & 12 & 1332 \\
$8 \mathrm{MeV}$ & $10 \times 10 \mathrm{~cm}^{2}$ & 120 & 493 \\
& $20 \times 20 \mathrm{~cm}^{2}$ & 60 & 499 \\
& $40 \times 40 \mathrm{~cm}^{2}$ & 12 & 709 \\
$12 \mathrm{MeV}$ & $10 \times 10 \mathrm{~cm}^{2}$ & 20 & 191 \\
& $20 \times 20 \mathrm{~cm}^{2}$ & 12 & 177 \\
& $40 \times 40 \mathrm{~cm}^{2}$ & 12 & 324 \\
$18 \mathrm{MeV}$ & $10 \times 10 \mathrm{~cm}^{2}$ & 20 & 390 \\
& $20 \times 20 \mathrm{~cm}^{2}$ & 12 & 343 \\
& $40 \times 40 \mathrm{~cm}^{2}$ & 12 & 455 \\
$22 \mathrm{MeV}$ & $10 \times 10 \mathrm{~cm}^{2}$ & 20 & 475 \\
$20 \times 20 \mathrm{~cm}^{2}$ & 12 & 334 \\
\hline \hline
\end{tabular}

energy, number of histories, field size and scoring field size. Typically, for an 18 
$\mathrm{MeV}$ electron beam, $10 \times 10 \mathrm{~cm}^{2}$ field size, and 20 million incident electrons there are approximately 14 million particles stored in the phase space file for a scoring field of radius $30 \mathrm{~cm}$. For the Pentium $2.4 \mathrm{GHz}$ processor, as a rough estimate, it takes an hour to perform a simulation with 5 million incident electrons (or histories).

Sufficient histories were followed to achieve better than $1 \%$ precision in the calculation of dose distributions for the Elekta SL25 electron beams listed in Table 4.2. For depth-dose simulation results compared to measurements for electron beams, please refer to Section 2.5 .

\subsection{BEAMDP calculations}

The BEAMDP code has been used to analyze and create multiple-source models. The following input parameters must be specified when processing data for the multiplesource beam models:

- Number of sub-sources

- Geometric details

- Type of field, number of bins, size of treatment/scoring field for fluence scoring

- Number of energy regions, energy bins, minimum energy, maximum energy

- Phase-space file to use

The number of sub-sources can be in the range between 1 and 22. Particles of different charges are considered to be from different sub-sources. There are a variety of sub-sources with respect to the components in an accelerator as was mentioned in

\subsection{BEAMDP CALCULATIONS}


Chapter 3. A basic model is a model with only two point sub-sources, one source for photons and one source for electrons. The effective source-surface distance, SSD, for each point source is calculated in the BEAMDP code by the pinhole method explained in Section 3.5. For the more complex models which include other elements in the beam, geometry details must be specified. The scattering foils, monitor chamber and mirror are simulated using plane sources at several positions along the beam axis. Particles from conical primary collimators are simulated by an annular source. Similarly, particles from secondary collimators or jaws are simulated by parallel bars. Applicators are simulated by rectangular rings.

Parameters stored in the model are the number of energy bins, relative fluence for each energy bin, and minimum and maximum energies. The minimum and maximum energies correspond to the minimum and maximum energies in the phase-space file. The number of bins is determined by the desired resolution. For example, if one want the uncertainty in the calculated depth of $50 \%$ dose, $R_{50}$, to be less than $1 \%$, the uncertainty in the peak position of the energy spectrum should be within $1 \%$ and therefore the bin width should be smaller than $1 \%$ of the peak energy. For the 12 $\mathrm{MeV}$ beam 128 bins were used with a bin width less than $0.1 \mathrm{MeV}$.

The planar fluence distribution for each sub-source is recorded on the scoring plane using the square field grid scheme (Figure 3.2, page 49). It includes the treatment and scoring field dimensions, the number of bins and the relative intensity of each bin - for each sub-source.

\subsection{DOSXYZNRC CALCULATIONS}




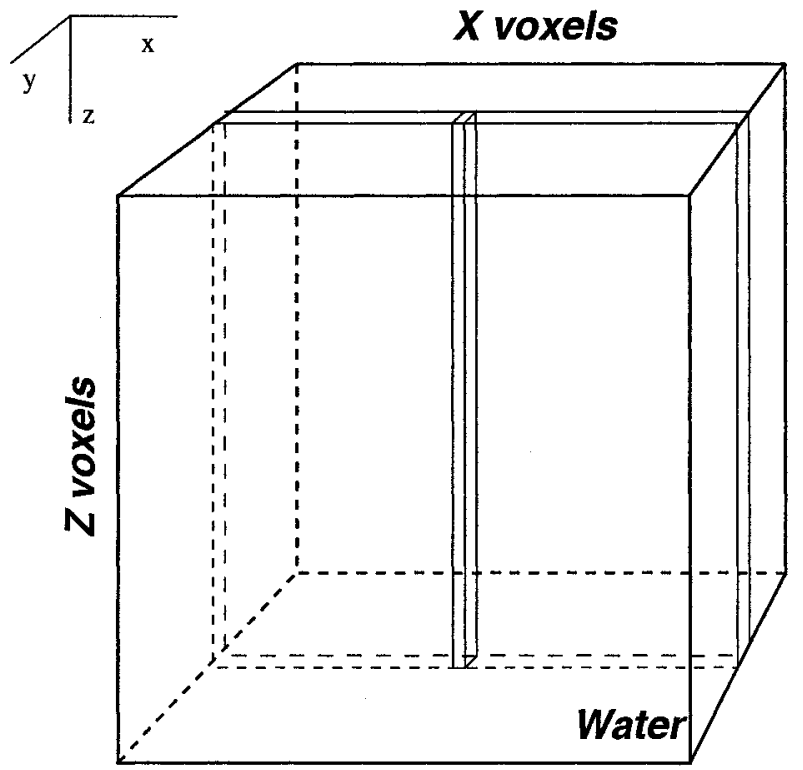

Figure 4.4: 3-D rectilinear water phantom

\subsection{DOSXYZnrc calculations}

The geometry used for the DOSXYZnre calculations is shown in Figure 4.4. Since much of the time in a DOSXYZnrc simulation is taken transporting particles to and away from voxel boundaries, only a portion of the phantom is divided into voxels. The rest of the phantom is essentially one region of the same medium defined using the parameter dsurround. ${ }^{51}$ The phantom is divided into $\mathrm{N}_{x} \mathrm{x} 1 \mathrm{xN}_{z}$ slices in the $\mathrm{x}, \mathrm{y}$, and $\mathrm{z}$ directions, respectively (Tables 4.3 and 4.4), resulting in a different number of voxels depending on energy and field size, ranging from $41 \times 1 \times 40=1640$ voxels for the $4 \mathrm{MeV}$ electron beam, $10 \times 10 \mathrm{~cm}^{2}$ field size to $61 \times 1 \times 56=3416$ voxels for the $22 \mathrm{MeV}$ electron beam and $40 \times 40 \mathrm{~cm}^{2}$ field size with no applicators. Voxel volume ranges from $0.1 \mathrm{~cm}^{3}$ to $0.25 \mathrm{~cm}^{3}$. In general, dose penetrates with deeper depths with the

\subsection{DOSXYZNRC CALCULATIONS}


energy and the CPU simulation time increases as the number of voxels increases. The unequal divisions are to minimize the total number of voxels while maintaining good resolution for lower energies. Central-axis depth-dose curves are calculated in the $1 \times 1 \mathrm{~cm}^{2}$ region around the central axis. Dose-profile curves are calculated in $41 \times 1$ $\mathrm{cm}^{2}$ region for $10 \times 10 \mathrm{~cm}^{2}$ field sizes, and in $61 \times 1 \mathrm{~cm}^{2}$ region for wider field sizes, $40 \times 40 \mathrm{~cm}^{2}$ and $20 \times 20 \mathrm{~cm}^{2}$ without applicators. The thickness of the phantom is 30 $\mathrm{cm}$ for photon beams, while it varies with energy for electron beams.

In this work, DOSXYZnrc is used to calculate dose for either original or reconstructed phase-space files and from multiple-source models using the following source options in DOSXYZnrc, respectively: ISOURCE=2, "Phase-Space Source Incident from Any Direction" and ISOURCE $=4$ : "Beam Characterization Model Incident from Any Direction". Calculations using the DOSXYZnrc code are run on single CPU. It usually takes between 15 and 25 minutes for the electron beams and between 30 and 40 minutes for the photon beams to get statistics better than $1 \%$ on a Pentium $2.4 \mathrm{GHz}$ processor. If DOSXYZnrc is used for dose calculations from phase-space files recycling is less than 4 times for all electron beams energies and less than 10 times for all photon beams (Section 1.4, page 16). For dose calculations from models recycling is not applicable. The statistical uncertainties in dose at the depth of maximum ionization and at a depth of $10 \mathrm{~g} / \mathrm{cm}^{2}$ are typically better than $1.0 \%$. The dose values are normalized to 1.0 at the depth of maximum dose, $d_{\max }$.

\subsection{Phase-space reconstruction}

Source models are built from phase-space files. To reconstruct a phase-space file from the model, the BEAMnrc code must be used with the source option ISOURC $=31$

\subsection{PHASE-SPACE RECONSTRUCTION}


Table 4.3: Number of voxels along x-axis $\left(N_{x}\right)$, z-axis $\left(N_{z}\right)$ and voxel sizes for various photon beam energies and field sizes. Along the y-axis one voxel has a size of $1 \mathrm{~cm}$.

\begin{tabular}{llllll}
\hline \hline Energy & Field size & Size x $N_{x}$ & Max X & Size x $N_{z}$ & Max Z \\
\hline $6 \mathrm{MV}$ & $10 \times 10 \mathrm{~cm}^{2}$ & $1 \mathrm{~cm} \times 41$ & $41 \mathrm{~cm}$ & $0.3 \mathrm{~cm} \times 100$ & $30 \mathrm{~cm}$ \\
& $40 \times 40 \mathrm{~cm}^{2}$ & $1 \mathrm{~cm} \times 61$ & $61 \mathrm{~cm}$ & $0.3 \mathrm{~cm} \times 100$ & $30 \mathrm{~cm}$ \\
$10 \mathrm{MV}$ & $10 \times 10 \mathrm{~cm}^{2}$ & $1 \mathrm{~cm} \times 41$ & $41 \mathrm{~cm}$ & $0.3 \mathrm{~cm} \times 100$ & $30 \mathrm{~cm}$ \\
& $40 \times 40 \mathrm{~cm}^{2}$ & $1 \mathrm{~cm} \mathrm{\times 61}$ & $61 \mathrm{~cm}$ & $0.3 \mathrm{~cm} \times 100$ & $30 \mathrm{~cm}$ \\
$25 \mathrm{MV}$ & $10 \times 10 \mathrm{~cm}^{2}$ & $1 \mathrm{~cm} \times 41$ & $41 \mathrm{~cm}$ & $0.3 \mathrm{~cm} \times 100$ & $30 \mathrm{~cm}$ \\
& $40 \times 40 \mathrm{~cm}^{2}$ & $1 \mathrm{~cm} \times 61$ & $61 \mathrm{~cm}$ & $0.3 \mathrm{~cm} \times 100$ & $30 \mathrm{~cm}$ \\
\hline \hline
\end{tabular}

(Phase Space Reconstructed Using Beam Models). Using this source option beam data are derived from a beam characterization model using the same sampling procedure described in Section 3.6. The only component module (CM) in the simulation input file needed for phase-space reconstruction is a SLAB CM with a thin layer of vacuum. Particles derived from a beam characterization model are incident on the vacuum layer. The thickness of the vacuum layer was set to $0.001 \mathrm{~cm}$. The phase-space file is created at the end of vacuum layer.

The CPU time to reconstruct the phase-space which contains the same number of particles as the original phase-space from the multiple-source model for the NRC Elekta SL25 $18 \mathrm{MeV}, 10 \times 10 \mathrm{~cm}^{2}$ field size, is about 214.0 seconds (0.059 hours) while the simulation for the original phase-space was 15700 seconds (about 4 hours). 
Table 4.4: Number of voxels along x-axis $\left(N_{x}\right)$, z-axis $\left(N_{z}\right)$ and voxel sizes for various electron beam energies and field sizes with or without (NA) applicators. Along the $\mathrm{y}$-axis one voxel has a size of $1 \mathrm{~cm}$.

\begin{tabular}{|c|c|c|c|c|c|}
\hline Energy & Field size & Size x $N_{x}$ & $\operatorname{Max} X$ & Size x $N_{z}$ & $\operatorname{Max} Z$ \\
\hline \multirow[t]{4}{*}{$4 \mathrm{MeV}$} & $10 \times 10 \mathrm{~cm}^{2}$ & $1 \mathrm{~cm} \times 41$ & $41 \mathrm{~cm}$ & $0.1 \mathrm{~cm} \mathrm{x} 40$ & $4 \mathrm{~cm}$ \\
\hline & $20 \times 20 \mathrm{~cm}^{2}$ & $1 \mathrm{~cm} \mathrm{x} 41$ & $41 \mathrm{~cm}$ & $0.1 \mathrm{~cm} \mathrm{x} 40$ & $4 \mathrm{~cm}$ \\
\hline & $20 \times 20 \mathrm{~cm}^{2} / \mathrm{NA}$ & $1 \mathrm{~cm} \times 61$ & $61 \mathrm{~cm}$ & $0.1 \mathrm{~cm} \times 40$ & $4 \mathrm{~cm}$ \\
\hline & $40 \times 40 \mathrm{~cm}^{2} / \mathrm{NA}$ & $1 \mathrm{~cm} \mathrm{x} 61$ & $61 \mathrm{~cm}$ & $0.1 \mathrm{~cm} \mathrm{x} 40$ & $4 \mathrm{~cm}$ \\
\hline \multirow[t]{4}{*}{$8 \mathrm{MeV}$} & $10 \times 10 \mathrm{~cm}^{2}$ & $1 \mathrm{~cm} \mathrm{x} 41$ & $41 \mathrm{~cm}$ & $0.15 \mathrm{~cm} \mathrm{x} 40$ & $6 \mathrm{~cm}$ \\
\hline & $20 \times 20 \mathrm{~cm}^{2}$ & $1 \mathrm{~cm} \times 41$ & $41 \mathrm{~cm}$ & $0.15 \mathrm{~cm} \mathrm{x} 40$ & $6 \mathrm{~cm}$ \\
\hline & $20 \times 20 \mathrm{~cm}^{2} / \mathrm{NA}$ & $1 \mathrm{~cm} \times 61$ & $61 \mathrm{~cm}$ & $0.15 \mathrm{~cm} \mathrm{x} 40$ & $6 \mathrm{~cm}$ \\
\hline & $40 \times 40 \mathrm{~cm}^{2} / \mathrm{NA}$ & $1 \mathrm{~cm} \times 61$ & $61 \mathrm{~cm}$ & $0.15 \mathrm{~cm} \mathrm{x} 40$ & $6 \mathrm{~cm}$ \\
\hline \multirow[t]{4}{*}{$12 \mathrm{MeV}$} & $10 \times 10 \mathrm{~cm}^{2}$ & $1 \mathrm{~cm} \mathrm{x} 41$ & $41 \mathrm{~cm}$ & $0.25 \mathrm{~cm} \mathrm{x} 40$ & $10 \mathrm{~cm}$ \\
\hline & $20 \times 20 \mathrm{~cm}^{2}$ & $1 \mathrm{~cm} \times 41$ & $41 \mathrm{~cm}$ & $0.25 \mathrm{~cm} \mathrm{x} 40$ & $10 \mathrm{~cm}$ \\
\hline & $20 \times 20 \mathrm{~cm}^{2} / \mathrm{NA}$ & $1 \mathrm{~cm} \times 61$ & $61 \mathrm{~cm}$ & $0.25 \mathrm{~cm} \mathrm{x} 40$ & $10 \mathrm{~cm}$ \\
\hline & $40 \times 40 \mathrm{~cm}^{2} / \mathrm{NA}$ & $1 \mathrm{~cm} \times 61$ & $61 \mathrm{~cm}$ & $0.25 \mathrm{~cm} \times 40$ & $10 \mathrm{~cm}$ \\
\hline \multirow[t]{4}{*}{$18 \mathrm{MeV}$} & $10 \times 10 \mathrm{~cm}^{2}$ & $1 \mathrm{~cm} \mathrm{x} 41$ & $41 \mathrm{~cm}$ & $0.25 \mathrm{~cm} \times 50$ & $12.5 \mathrm{~cm}$ \\
\hline & $20 \times 20 \mathrm{~cm}^{2}$ & $1 \mathrm{~cm} \mathrm{x} 41$ & $41 \mathrm{~cm}$ & $0.25 \mathrm{~cm} \mathrm{x} 50$ & $12.5 \mathrm{~cm}$ \\
\hline & $20 \times 20 \mathrm{~cm}^{2} / \mathrm{NA}$ & $1 \mathrm{~cm} \mathrm{x} 61$ & $61 \mathrm{~cm}$ & $0.25 \mathrm{~cm} \times 50$ & $12.5 \mathrm{~cm}$ \\
\hline & $40 \times 40 \mathrm{~cm}^{2} / \mathrm{NA}$ & $1 \mathrm{~cm} \times 61$ & $61 \mathrm{~cm}$ & $0.25 \mathrm{~cm} \mathrm{x} 50$ & $12.5 \mathrm{~cm}$ \\
\hline \multirow[t]{4}{*}{$22 \mathrm{MeV}$} & $10 \times 10 \mathrm{~cm}^{2}$ & $1 \mathrm{~cm} \mathrm{x} 41$ & $41 \mathrm{~cm}$ & $0.25 \mathrm{~cm} \times 56$ & $14 \mathrm{~cm}$ \\
\hline & $20 \times 20 \mathrm{~cm}^{2}$ & $1 \mathrm{~cm} \mathrm{x} 41$ & $41 \mathrm{~cm}$ & $0.25 \mathrm{~cm} \mathrm{x} 56$ & $14 \mathrm{~cm}$ \\
\hline & $20 \times 20 \mathrm{~cm}^{2} / \mathrm{NA}$ & $1 \mathrm{~cm} \mathrm{x} 61$ & $61 \mathrm{~cm}$ & $0.25 \mathrm{~cm} \times 56$ & $14 \mathrm{~cm}$ \\
\hline & $40 \times 40 \mathrm{~cm}^{2} / \mathrm{NA}$ & $1 \mathrm{~cm} \times 61$ & $61 \mathrm{~cm}$ & $0.25 \mathrm{~cm} \times 56$ & $14 \mathrm{~cm}$ \\
\hline
\end{tabular}




\subsection{Calculation results}

In this chapter, calculation results of BEAMnrc simulations from the NRC Elekta SL25 photon and electron beams were presented, and the difference between centralaxis depth-dose values calculated from the phase-space file and ones from the experiment were compared

The main objective of BEAMnrc calculations is to generate a variety of phasespace data files that are used as input source files for the DOSXYZnrc program to calculate dose distributions in a water phantom, and to create a basis on which the multiple-source models can be built. Therefore, the most significant calculation results in this project are for the dose calculation from the models that will be presented after models are analyzed and created in the next two chapters, and used in DOSXYZnrc calculations. Regardless of the $5 \%(10 \%$ in $4 \mathrm{MeV}$ beam) disagreement in the buildup region in the DOSXYZnrc electron dose calculations, as it was already addressed in Chapter 2, the set of phase-space files obtained here are still satisfying our requirements for multiple-source beam models which will be discussed and studied in the next two chapters. 


\section{Chapter 5}

\section{Original phase space file analysis}

The general characteristics of the simulated photon and electron beams from the Elekta SL25 linear accelerator (see Figure 2.1), as calculated by BEAMnrc user code, were analyzed using the data processing BEAMDP utility, in order to emphasize some of the major characteristics of the beams before creating sub-source beam models.

\subsection{Electron beams}

Multiple-source beam models are built on phase-space files which are scored with comprehensive inherited LATCH bit setting (Section 3.1.1). Using LATCH bit filters with the BEAMDP code gives the ability to concentrate on the particles from individually selected accelerator components to investigate our component-based sub-source model.

The electron spectral distribution from a few accelerator components are 


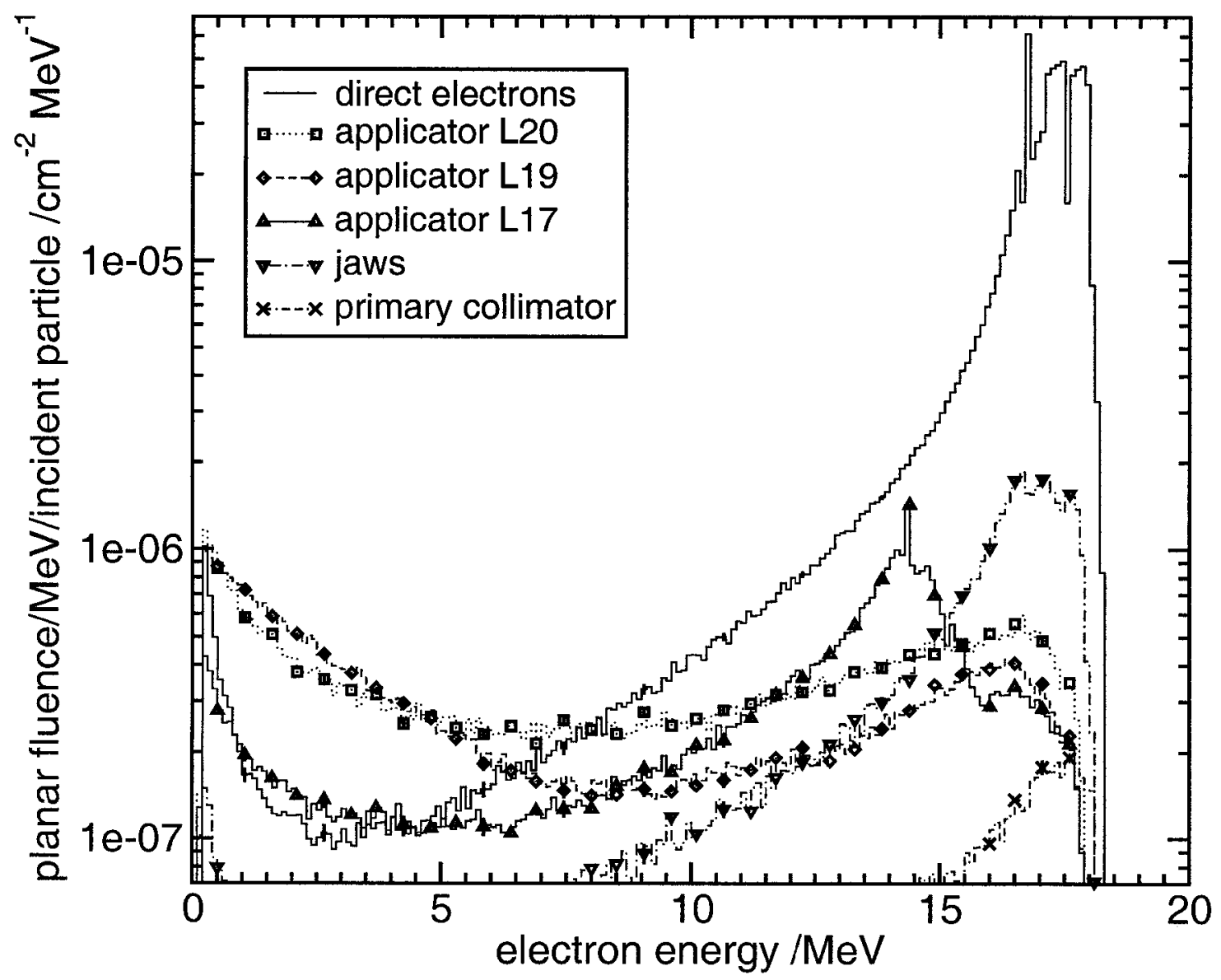

Figure 5.1: Spectra of the electron components of the Elekta SL25 $18 \mathrm{MeV}$ electron beam with $10 \times 10 \mathrm{~cm}^{2}$ treatment field at $100 \mathrm{~cm}$ nominal SSD. About $67 \%$ of electrons are "direct" electrons, those coming directly from the scattering foil without hitting any collimator or applicator components. "applicator L20" electrons are those which interacted in the applicator with LATCH bit 20 (bottom applicator), (see Figure 2.1, page 27). 
shown in Figure 5.1. If a particle interacts in two components then the particle is assigned to the closest component to the scoring plane. For example, an electron from the exit window with the LATCH bit 1 set, which also interacts in the jaws with LATCH bit 13, is considered as a "jaws" electron. The spectral distribution of the "direct" electrons (those coming directly from the exit window and scattering foils without hitting any of the beam modifying components like jaws or applicators) consists of a prominent peak around $17 \mathrm{MeV}$ and a long tail. These electrons are about $67 \%$ of the total electrons. About $6 \%$ of the total electrons in the beam are "jaws" electrons that reach the scoring plane directly after being scattered from the secondary collimator jaws. They have slightly lower energy than those that missed the jaws.

About $6 \%$ of the total electrons are scattered from "applicator L17". The spectral distribution of these electrons has two significant peaks where the number of electrons varies rapidly around 14 and $17 \mathrm{MeV}$. The change between 14 and $17 \mathrm{MeV}$ corresponds to electrons that traversed an upstream applicator ("applicator L16") and had minimal energy loss in "applicator L17", producing low-energy delta-rays or bremsstrahlung (the slight rise in fluence at the low-energy end of the spectrum indicates the presence of delta-rays). The change at $14 \mathrm{MeV}$ corresponds to electrons that are scattered at the upstream component, jaws or applicator L16, with significant energy loss. Many more low-energy delta-rays or bremsstrahlung are produced in "applicator L19" which is the most exposed applicator in the beam. A significant portion of the electrons from "applicator L20" are created by the bremsstrahlung photons created upstream. Electrons scattered at the downstream applicators all have substantial energy losses.

The electron planar fluence for each component is almost flat inside the

\subsection{ELECTRON BEAMS}


$10 \times 10 \mathrm{~cm}^{2}$ treatment field, with the exception of "applicator L20" electrons that show the largest variation across the field (Figure 5.2). The peak on "applicator L20" electrons around $4.5 \mathrm{~cm}$ is from electrons created by bremsstrahlung photons in the applicator and then reached the scoring plane located just a few centimeters downstream. Planar fluence for electrons from applicators upstream show a change

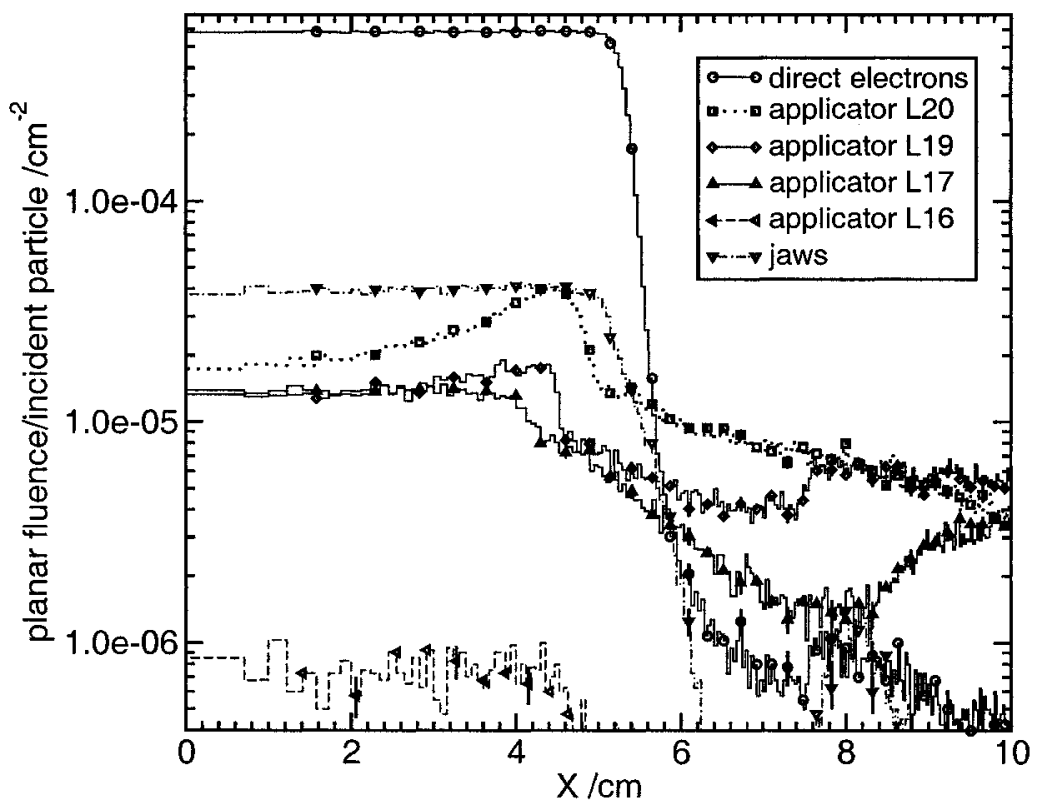

Figure 5.2: Planar fluence of electrons as a function of lateral position for the electron components of the NRC Elekta SL25 $18 \mathrm{MeV}$ electron beam with $10 \times 10 \mathrm{~cm}^{2}$ treatment field at $100 \mathrm{~cm}$ nominal SSD.

between 4 and $8 \mathrm{~cm}$ caused by the "shadowing" effect of downstream applicators.

Bremsstrahlung photons make up $82 \%$ of all particles in the $18 \mathrm{MeV}$ electron beam, 10x10 $\mathrm{cm}^{2}$ field size. The spectra of photons, shown in Figure 5.3(a) from different components of the accelerator have very similar characteristics as those of electrons in a sense that "direct" photons and those coming from the components close

\subsection{ELECTRON BEAMS}


to the scoring plane are the most significant. The fluence decreases with increasing photon energy in the spectra. The spectra are weakly dependent on the particle position inside the treatment field, as shown on Figure 5.4(a) and 5.4(b) for electron and photon mean energies respectively, as a function of distance off axis in square rings. The spectral distribution varies considerably more with position outside the treatment field, but the fluence is low in this region.

The photons originating in the two bottom applicators and "direct" photons are the three most significant photon components (Figure 5.3(b)). Just a few photons that interacted at the primary collimator reach the scoring plane.

The planar fluence for the "applicator L20" photons (Figure 5.3(b)) has a similar peak as the electrons from the same applicator, except slightly further out under the applicator's bars. For other two upstream applicators, a "shadowing" effect can be observed similar to the electron beam case.

There is a strong correlation between energy, direction, position, and particle origin. Direct electrons generally emerge cleanly from the treatment head with higher energies and with a narrow angular distribution, centered about the diverging ray from the effective focal spot on the beam axis to the area of the treatment field.

The mean energy of particles inside the treatment field is approximately constant for "direct" particles but generally varies for all other types, as shown in Figures 5.4(a) and 5.4(b). The biggest variation for electrons inside the treatment field is observed for "jaws" electrons peaked between 4 and $5 \mathrm{~cm}$ distance off axis, for the scattered electrons with the lowest energy loss and change in direction, passing close to applicators without interacting with them. Outside the treatment field the mean energy varies significantly for all types of particles.

\subsection{ELECTRON BEAMS}



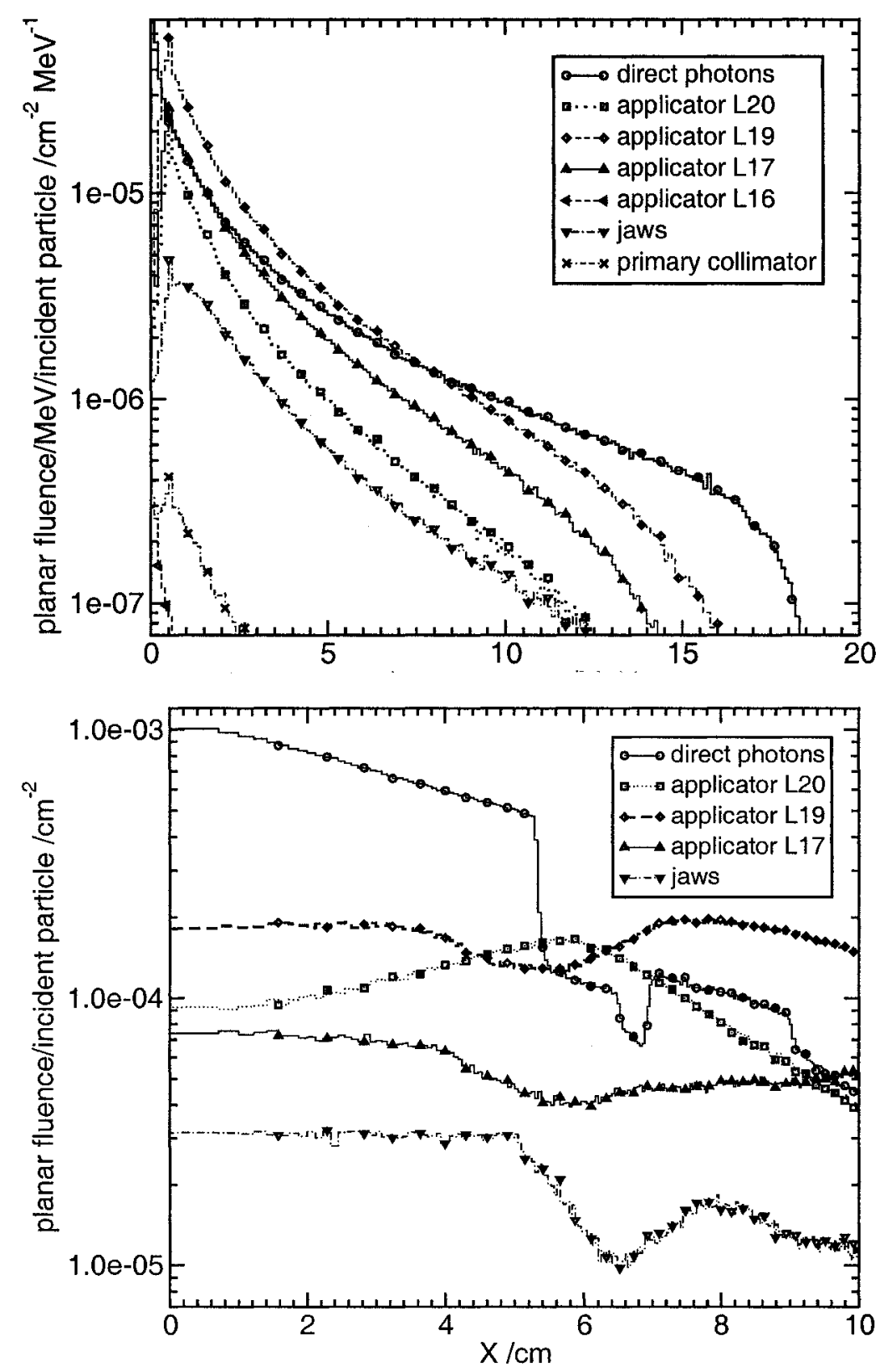

Figure 5.3: (a) Spectra of the photon components of the NRC Elekta SL25 $18 \mathrm{MeV}$ electron beam with $10 \times 10 \mathrm{~cm}^{2}$ treatment field at $100 \mathrm{~cm}$ nominal SSD. About $30 \%$ of total photons are "applicator L19" photons, 20\% are "applicator L19" $15 \%$ are "direct" photons, those coming directly from the scattering foil without interacting in any of beam collimating components. "jaws" photons are those which interacted in the jaws with LATCH bit 13 or L14, etc.

(b) Planar fluence of photons as a function of lateral position in square rings on axis for the photon components of the NRC Elekta SL25 $18 \mathrm{MeV}$ electron beam with $10 \times 10 \mathrm{~cm}^{2}$ treatment field at $100 \mathrm{~cm}$ nominal SSD.

\subsection{ELECTRON BEAMS}



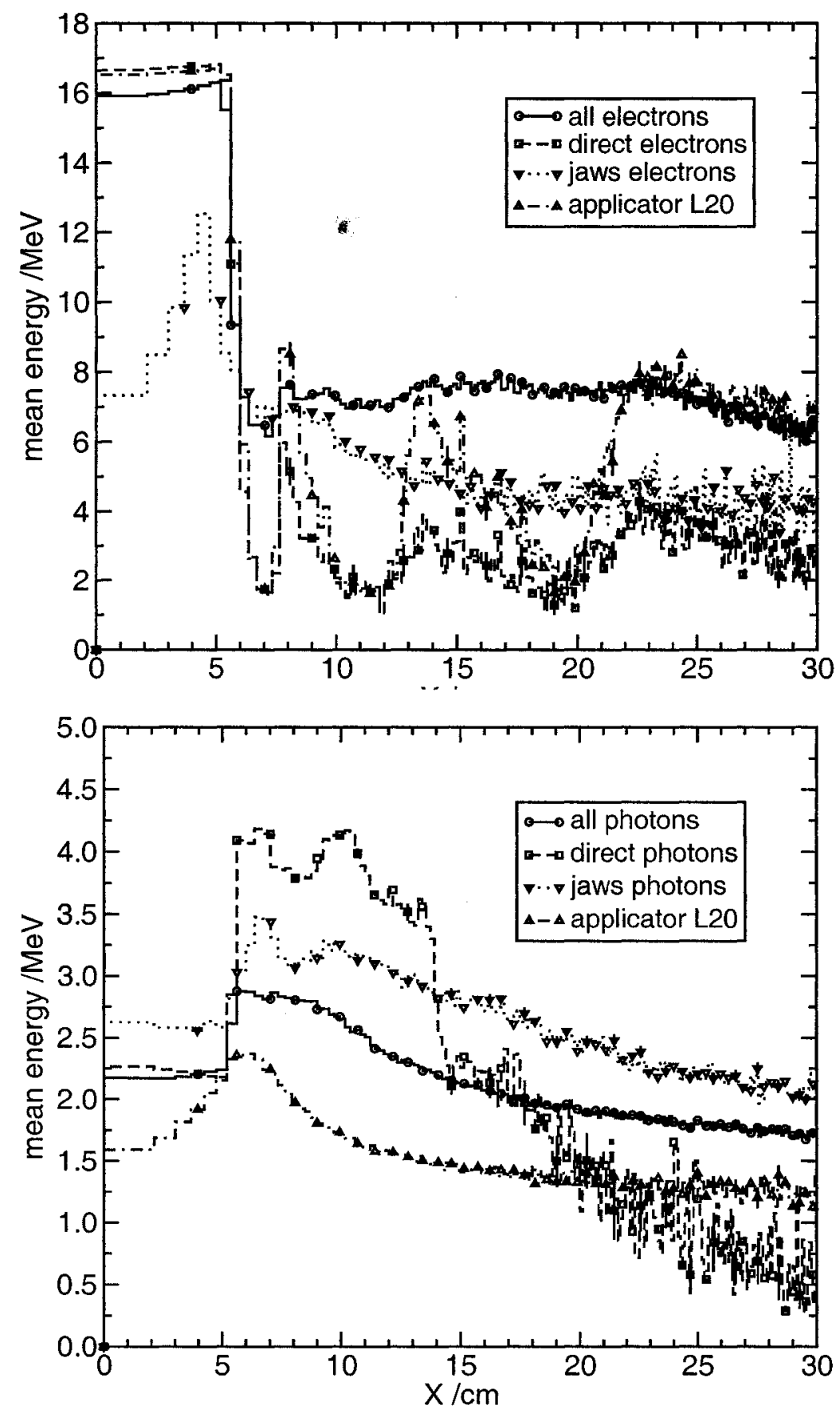

Figure 5.4: (a) Electron mean energies as a function of distance off axis in square rings from the NRC Elekta SL25 $18 \mathrm{MeV}$ electron beam with $10 \times 10 \mathrm{~cm}^{2}$ treatment field at $100 \mathrm{~cm}$ nominal SSD.

(b) Photon mean energies as a function of distance off axis in square rings from the NRC Elekta SL25 $18 \mathrm{MeV}$ electron beam with $10 \times 10 \mathrm{~cm}^{2}$ treatment field at $100 \mathrm{~cm}$ nominal SSD.

\subsection{ELECTRON BEAMS}




\subsection{Photon beams}

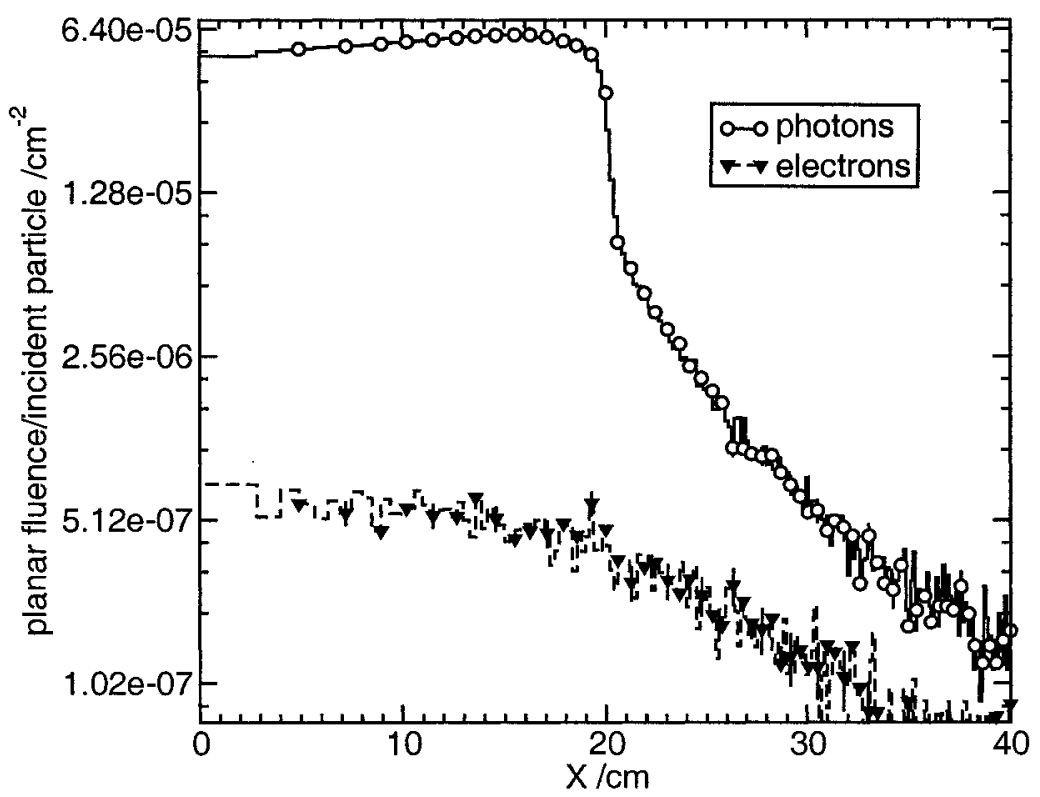

Figure 5.5: Planar fluence of photons and electrons from the NRC Elekta SL25 25 MV photon beam with $40 \times 40 \mathrm{~cm}^{2}$ treatment field at $100 \mathrm{~cm}$ nominal SSD.

The analysis of the photon beams from the NRC Elekta SL25 gives similar characteristics to the electron beam. The total number of "direct" photons is about $95 \%$ of the total photon fluence. The rest of the photons are those that interacted in the collimator and flattening filter.

Planar fluence as a function of lateral position for the $25 \mathrm{MV} 40 \times 40 \mathrm{~cm}^{2}$ photon beam is given in Figure 5.5 which shows that fluence is almost flat for the photons inside the treatment field, but changes slowly outside the treatment field. However, the planar fluence of electrons is changing continuously across the whole scoring field.

Only about $2 \%$ of the particles are electrons in the $25 \mathrm{MV}$ photon beam, but 
their contribution to absorbed dose is significant as will be presented in the next chapter. The majority of the electrons, about $80 \%$, are electrons created in the air and electrons from the flattening filter. The rest of the electrons, about $4 \%$, are scattered at the jaws of the linear accelerator (Figure 5.6). All the electrons created in the air from the photons that are coming directly from the target have LATCH bit 1 inherited and they appear as "direct" electrons according to the LATCH bit setting. For example, a photon from the target which has LATCH bit 1 does not interact with any linac's component, but creates an electron in the air between the mylar screen and scoring plane. That electron inherits LATCH bit 1 from the photon and have LATCH bit 23 set, which is used for the air. Since the air is neither component of linear accelerator nor sub-source in multiple-source model the electron appears as "direct".

The mean energy of particles varies across the scoring field as shown in Figure 5.7. The approximation that the mean energy is flat inside and outside the treatment field as assumed in the old BEAMDP code will affect the depth-dose calculation from the model since in the beam reconstruction routine the energy of the particle will be calculated using these values. Having more energy regions and the mean energy for each particular region as was already shown in Figure 3.4, page 54, the improved beam reconstruction brings more accuracy to the depth-dose calculation from beam models as it will be discussed in the next Chapter.

\subsection{PHOTON BEAMS}




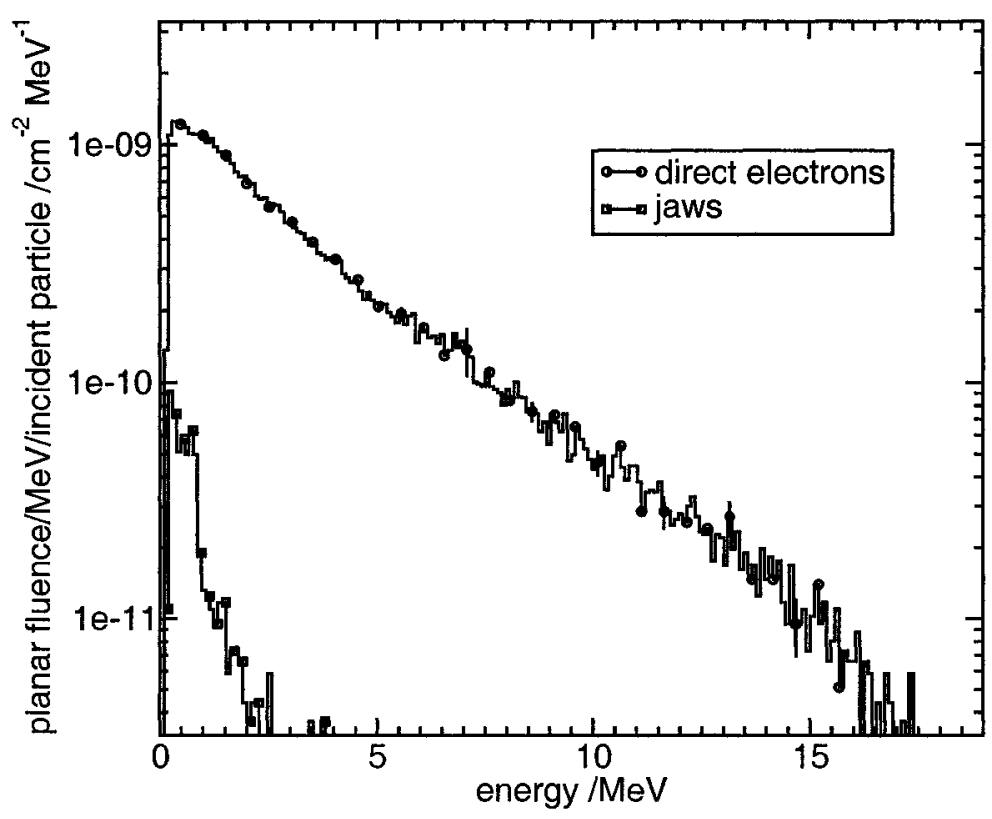

Figure 5.6: Spectra of the electron components of the NRC Elekta SL25 25 MV photon beam with $10 \times 10 \mathrm{~cm}^{2}$ treatment field at $100 \mathrm{~cm}$ nominal SSD. The majority of electrons are "direct" electrons (about 80\%), and the rest are from the jaws and other components.

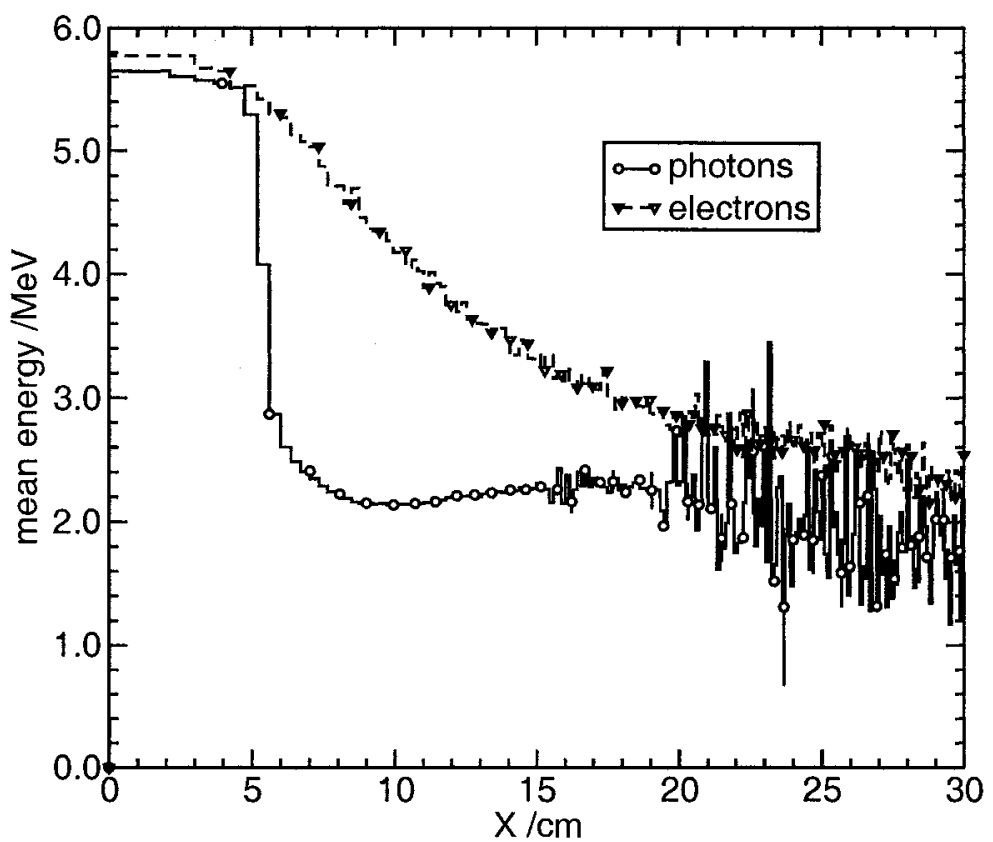

Figure 5.7: Photon and electron mean energies of the NRC Elekta SL25 25 MV photon beam with $10 \times 10 \mathrm{~cm}^{2}$ treatment field at $100 \mathrm{~cm}$ nominal SSD. The large uncertainty for the mean energy beyond $20 \mathrm{~cm}$ is due to DBS variance reduction method.

\subsection{PHOTON BEAMS}




\subsection{Conclusion}

For the analysis of the original phase-space files, the $18 \mathrm{MeV}$ electron beam and $25 \mathrm{MV}$ photon beam were used. The same results in terms of planar fluence, energy distribution and mean energy can be obtained for the other electron or photon beams.

"Direct" particles represent a majority of the particles in electron or photon beams. They should be included as the "direct" sources for both types of particles, photons and electrons. Usually, there are few positrons in the beam, so that a "direct" sub-source of positrons may be omitted. More than $25 \%$ of the particles in the beam arise from the collimators and applicators which have specific spectral and spatial characteristics. These sub-sources should also be included in the model.

Although $82 \%$ of particles in the $18 \mathrm{MeV}$ electron beam are photons, their contribution to the absorbed dose is much less than the contribution from the electrons (the photons contribution is just a few percent of $D_{\max }$ ). Thus the number of photon sub-sources for the electron beam can be reduced to only the most significant ones.

Only $2 \%$ of particles in the $25 \mathrm{MV}$ photon beam are electrons. Their contribution to the absorbed dose is not negligible. At least one source of electrons must be included in the model. Electron sources should be included only for the beam modifying components, like applicators and jaws, but not for components traversed by each electron that reaches the scoring plane (mirror, mylar screen, etc.)

The mean energy distribution is also an important characteristic of the beam. The old BEAMDP code assumed that the mean energy distribution is constant either inside or outside the treatment field. This is generally not a good approximation, and the scoring field should be divided into more than than two regions.

\subsection{CONCLUSION}




\section{Chapter 6}

\section{Models and results}

A variety of multiple-source models have been developed during the course of this work:

1. full model including positron source

2. full model, electron-photon sub-sources only for electron beams, and electronpositron-photon more significant sub-sources for photon beams

3. reduced model, only significant components

4. optimized model, two point sources plus two most significant components for electron beams, one point source, two air components plus two most significant components for photon beams

5. two-source model, two "direct" sources, for electrons and photons

Models differ in their complexity. The full model contains the highest number of components, which is reduced for each subsequent model, so that the two-source 
model contains only 2 components for electron beams, and the three-source model only 3 components for photon beams . Basically, the total number of sub-sources is the only difference among these models, since parameters required by the BEAMDP code for each model are the same for any particular combination of beam type, energy and field size. Parameters for the NRC Elekta SL25 $18 \mathrm{MeV}$ electron beam and the $25 \mathrm{MV}$ photon beam, 10x10 $\mathrm{cm}^{2}$ field size, are listed in Table 6.1.

The full model contains photon and electron and sub-sources for all components in the linear accelerator model, including one point source for positrons. The number of positrons in phase-space files for electron beams is usually very small and positron sources may be generally omitted from the model. However, the number of positrons in photon beams could be significant, so that a positron sub-source must be maintained in all models. For the electron beams, in the second full model, only electron-photon sources were considered, positrons are ignored (not scored). Since some sources in the full electron-photon model have very low intensity, the model can be easily reduced by discarding these sources. All particles that originally belong to the discarded source are assigned to the next upstream source of the same particle type in the model. The reduced model contains only significant sources. However, in the optimized model for the electron beam, the number of sources is further reduced to just four sources, two point ("direct") sources and two others - the most significant sources. Finally, the two-source model contains only two point sources, one for each of the particles, photons and electrons.

In this chapter, the difference in dose calculations for all these models from the NRC Elekta SL25 $18 \mathrm{MeV}$ electron beam and $25 \mathrm{MV}$ photon beam are presented. 
Table 6.1: Parameters used in multiple-source model creation for the Elekta SL25 $18 \mathrm{MeV}$ electron beam and $25 \mathrm{MV}$ photon beam, $10 \times 10 \mathrm{~cm}^{2}$ field size. Square field type is applied for all models.

\begin{tabular}{|c|c|c|}
\hline Parameter & $18 \mathrm{MeV}$ & $25 \mathrm{MV}$ \\
\hline Number of energy bins & 164 & 186 \\
\hline$E_{m i n}$ & $0.01 \mathrm{MeV}$ & $0.01 \mathrm{MeV}$ \\
\hline$E_{\max }$ & $22.36 \mathrm{MeV}$ & $18.998 \mathrm{MeV}$ \\
\hline Number of energy regions & 10 & 12 \\
\hline \multirow[t]{12}{*}{ Half-width of energy region } & $2.5 \mathrm{~cm}$ & $2.0 \mathrm{~cm}$ \\
\hline & $4.5 \mathrm{~cm}$ & $3.0 \mathrm{~cm}$ \\
\hline & $5.0 \mathrm{~cm}$ & $4.0 \mathrm{~cm}$ \\
\hline & $5.5 \mathrm{~cm}$ & $4.5 \mathrm{~cm}$ \\
\hline & $6.0 \mathrm{~cm}$ & $5.0 \mathrm{~cm}$ \\
\hline & $6.5 \mathrm{~cm}$ & $5.5 \mathrm{~cm}$ \\
\hline & $7.0 \mathrm{~cm}$ & $6.0 \mathrm{~cm}$ \\
\hline & $7.5 \mathrm{~cm}$ & $6.5 \mathrm{~cm}$ \\
\hline & $8.5 \mathrm{~cm}$ & $7.0 \mathrm{~cm}$ \\
\hline & $10.0 \mathrm{~cm}$ & $8.0 \mathrm{~cm}$ \\
\hline & & $10.0 \mathrm{~cm}$ \\
\hline & & $15.0 \mathrm{~cm}$ \\
\hline Actual half-width size of treatment field & $5 \mathrm{~cm}$ & $5 \mathrm{~cm}$ \\
\hline Half-width size of greater treatment field area & $6 \mathrm{~cm}$ & $6 \mathrm{~cm}$ \\
\hline Half-width size of scoring field & $10 \mathrm{~cm}$ & $15 \mathrm{~cm}$ \\
\hline Number of spatial bins inside the treatment field & 40 & 40 \\
\hline Number of spatial bins outside the treatment field & 20 & 20 \\
\hline
\end{tabular}




\subsection{Electron beam}

\subsubsection{Models}

Components of the full multiple-source model for the NRC Elekta SL25 $18 \mathrm{MeV}$ electron beam, $10 \times 10 \mathrm{~cm}^{2}$ field size, are presented in Table 6.2. There are a total of 21 components, 7 for electrons, 13 for photons and 1 for positrons. Three point sources for electrons, photons and positrons are source number 1, 2 and 3, respectively. For each point source the distance (d) from the scoring plane is calculated by the pinhole method in BEAMDP as explained in Section 3.5. The distance calculated for positrons, $167 \mathrm{~cm}$, indicates very narrow angular distribution for this particles. Other electron sources in this model are from jaws with LATCH bits 13 and 14, and applicators with LATCH bits 16, 17, 19 and 20. Relative intensity, i.e. number of particles from the sub-source divided by the total number of particles (RI) and relative intensity per particle type (PRI) are given in the table for each sub-source.

Only $0.16 \%$ of the particles are positrons in the $18 \mathrm{MeV}$ electron beam and they can be totally omitted from the model. The effect of this change in dose calculations may be observed on Figures 6.1 and 6.2. By dropping the positron source the model is reduced to electron and photon sources only, which causes a slight change in relative intensity for the other sub-sources (Table 6.3).

Components for the reduced model are given in Table 6.4. The following 6 weak photon sources have been eliminated: monitor chamber, two scattering foils, collimator, mirror and mylar screen. The remaining components, besides point sources, are those closer to the scoring plane, collimators and applicators. A central-axis depth-dose comparison for this model against the original phase-space file is shown

\subsection{ELECTRON BEAM}


Table 6.2: Components of the full multiple-source model for the NRC Elekta SL25 $18 \mathrm{MeV}$ electron beam, $10 \times 10 \mathrm{~cm}^{2}$ field size. Ch is charge of the sub-source, $\mathrm{L}$ is LATCH bit, $d$ is the distance from the sub-source to the scoring plane, 'PRI' is relative intensity per particle type. The model contains 21 sources, 7 for electrons, 13 for photons and 1 for positrons.

\begin{tabular}{|c|c|c|c|c|c|c|c|}
\hline Source & Source type & Component & $\mathrm{Ch}$ & $\mathrm{L}$ & $\mathrm{d} / \mathrm{cm}$ & $\mathrm{RI} / \%$ & PRI $/ \%$ \\
\hline 1 & Point source & Exit window & -1 & 1 & 98 & 21.22 & 82.18 \\
\hline 2 & Point source & Exit window & 0 & 1 & 99 & 9.81 & 13.26 \\
\hline 3 & Point source & Exit window & 1 & 1 & 167 & 0.16 & 100.00 \\
\hline 4 & Circ.plane & Scattering foil & 0 & 2 & 99 & 10.81 & 14.61 \\
\hline 5 & Ring & Collimator & 0 & 4 & 93 & 0.25 & 0.33 \\
\hline 6 & Circ.plane & Scattering foil & 0 & 5 & 85 & 9.06 & 12.24 \\
\hline 7 & Circ.plane & Ion chamber & 0 & 7 & 83 & 0.12 & 0.16 \\
\hline 8 & Rect.plane & Mirror & 0 & 9 & 77 & 0.27 & 0.37 \\
\hline 9 & Collimator & Jaws & -1 & 13 & 67 & 0.86 & 3.33 \\
\hline 10 & Collimator & Jaws & 0 & 13 & 67 & 1.26 & 1.70 \\
\hline 11 & Collimator & Jaws & -1 & 14 & 55 & 0.58 & 2.25 \\
\hline 12 & Collimator & Jaws & 0 & 14 & 55 & 1.06 & 1.43 \\
\hline 13 & Circ.plane & Mylar screen & 0 & 15 & 47 & 0.56 & 0.76 \\
\hline 14 & Applicator & 1st applicator & -1 & 16 & 46 & 0.03 & 0.11 \\
\hline 15 & Applicator & 1st applicator & 0 & 16 & 46 & 0.02 & 0.02 \\
\hline 16 & Applicator & 2nd applicator & -1 & 17 & 28 & 0.61 & 2.36 \\
\hline 17 & Applicator & 2nd applicator & 0 & 17 & 28 & 6.46 & 8.73 \\
\hline 18 & Applicator & 3rd applicator & -1 & 19 & 15 & 0.98 & 3.78 \\
\hline 19 & Applicator & 3rd applicator & 0 & 19 & 15 & 21.41 & 28.93 \\
\hline 20 & Applicator & 4th applicator & -1 & 20 & 7 & 1.55 & 6.00 \\
\hline 21 & Applicator & 4th applicator & 0 & 20 & 7 & 12.93 & 17.47 \\
\hline
\end{tabular}


Table 6.3: Components of the full electron-photon multiple-source model for $18 \mathrm{MeV}$ electron beam, $10 \times 10 \mathrm{~cm}^{2}$ field size. The model contains 20 sources, 7 for electrons and 13 for photons.

\begin{tabular}{llllllll}
\hline \hline Source & Source type & Component & Ch & L & d/cm & RI/\% & PRI/\% \\
\hline 1 & Point source & Exit window & -1 & 1 & 98 & 21.25 & 82.18 \\
2 & Point source & Exit window & 0 & 1 & 99 & 9.83 & 13.26 \\
3 & Circ plane & Scattering foil & 0 & 2 & 99 & 10.83 & 14.61 \\
4 & Ring & Collimator & 0 & 4 & 93 & 0.25 & 0.33 \\
5 & Circ. plane & Scattering foil & 0 & 5 & 85 & 9.08 & 12.24 \\
6 & Circ. plane & Ion chamber & 0 & 7 & 83 & 0.12 & 0.16 \\
7 & Rect. plane & Mirror & 0 & 9 & 76 & 0.27 & 0.37 \\
8 & Collimator & Jaws & -1 & 13 & 67 & 0.86 & 3.33 \\
9 & Collimator & Jaws & 0 & 13 & 67 & 1.26 & 1.70 \\
10 & Collimator & Jaws & -1 & 14 & 55 & 0.58 & 2.25 \\
11 & Collimator & Jaws & 0 & 14 & 55 & 1.06 & 1.43 \\
12 & Circ. plane & Mylar screen & 0 & 15 & 47 & 0.56 & 0.76 \\
13 & Applicator & 1st applicator & -1 & 16 & 46 & 0.03 & 0.11 \\
14 & Applicator & 1st applicator & 0 & 16 & 46 & 0.02 & 0.02 \\
15 & Applicator & 2nd applicator & -1 & 17 & 28 & 0.61 & 2.36 \\
16 & Applicator & 2nd applicator & 0 & 17 & 28 & 6.47 & 8.73 \\
17 & Applicator & 3rd applicator & -1 & 19 & 15 & 0.98 & 3.78 \\
\hline \hline
\end{tabular}


in Figure 6.1.

Table 6.4: Components of the reduced multiple-source model for $18 \mathrm{MeV}$ electron beam, $10 \times 10 \mathrm{~cm}^{2}$ field size. The model contains 14 sources, 7 for electrons and 7 for photons.

\begin{tabular}{llllllll}
\hline \hline Source & Source type & Component & Ch & L & d/cm & RI/\% & PRI/\% \\
\hline 1 & Point source & Exit window & -1 & 1 & 98 & 21.25 & 82.18 \\
2 & Point source & Exit window & 0 & 1 & 98 & 30.90 & 41.68 \\
3 & Collimator & Jaws & -1 & 13 & 67 & 0.86 & 3.33 \\
4 & Collimator & Jaws & 0 & 13 & 67 & 1.28 & 1.72 \\
5 & Collimator & Jaws & -1 & 14 & 55 & 0.58 & 2.25 \\
6 & Collimator & Jaws & 0 & 14 & 55 & 1.07 & 1.45 \\
7 & Applicator & 1st applicator & -1 & 16 & 46 & 0.03 & 0.11 \\
8 & Applicator & 1st applicator & 0 & 16 & 46 & 0.02 & 0.02 \\
9 & Applicator & 2nd applicator & -1 & 17 & 28 & 0.61 & 2.36 \\
10 & Applicator & 2nd applicator & 0 & 17 & 28 & 6.47 & 8.73 \\
11 & Applicator & 3rd applicator & -1 & 19 & 15 & 0.98 & 3.78 \\
12 & Applicator & 3rd applicator & 0 & 19 & 15 & 21.44 & 28.93 \\
13 & Applicator & 4th applicator & -1 & 20 & 7 & 1.55 & 6.00 \\
14 & Applicator & 4th applicator & 0 & 20 & 7 & 12.95 & 17.47 \\
\hline \hline
\end{tabular}

An even more simplified model can give a very good result in dose calculations. An optimized, four-source model is presented in Table 6.5 , which contains only a point source for electrons and one for photons and two applicator electron sources.

The most simplified model is one which contains only two sources, one point

\subsection{ELECTRON BEAM}


Table 6.5: Components of the optimized multiple-source model for $18 \mathrm{MeV}$ electron beam, $10 \times 10 \mathrm{~cm}^{2}$ field size. The model contains 4 sources, 3 for electrons and 1 for photons.

\begin{tabular}{lllccccc}
\hline \hline Source & Source type & Component & Ch & L & d/cm & RI/\% & PRI/\% \\
\hline 1 & Point source & Exit window & -1 & 1 & 98 & 23.33 & 90.22 \\
2 & Point source & Exit window & 0 & 1 & 98 & 74.14 & 100.00 \\
3 & Applicator & 3rd applicator & -1 & 19 & 15 & 0.98 & 3.78 \\
4 & Applicator & 4th applicator & -1 & 20 & 7 & 1.55 & 6.00 \\
\hline \hline
\end{tabular}

source for electrons and one point source for photons (Table 6.6).

Table 6.6: Components of the two-source model for $18 \mathrm{MeV}$ electron beam, $10 \times 10 \mathrm{~cm}^{2}$ field size. The model contains 2 sources, 1 for electrons and 1 for photons.

\begin{tabular}{llllllll}
\hline \hline Source & Source type & Component & $\mathrm{Ch}$ & $\mathrm{L}$ & $\mathrm{d} / \mathrm{cm}$ & $\mathrm{RI} / \%$ & $\mathrm{PRI} / \%$ \\
\hline 1 & Point source & Exit window & -1 & 1 & 98 & 25.86 & 100.00 \\
2 & Point source & Exit window & 0 & 1 & 98 & 74.14 & 100.00 \\
\hline \hline
\end{tabular}

\subsubsection{Depth-dose and dose-profile comparisons}

Central-axis depth-dose comparison for the Elekta SL25 $18 \mathrm{MeV}$ electron beam, $10 \times 10 \mathrm{~cm}^{2}$ field, calculated using original phase-space file and multiple-source models shows good agreement for all models (Figure 6.1).

Figure 6.2 compares various dose-profiles and it can be observed that agree-

\subsection{ELECTRON BEAM}




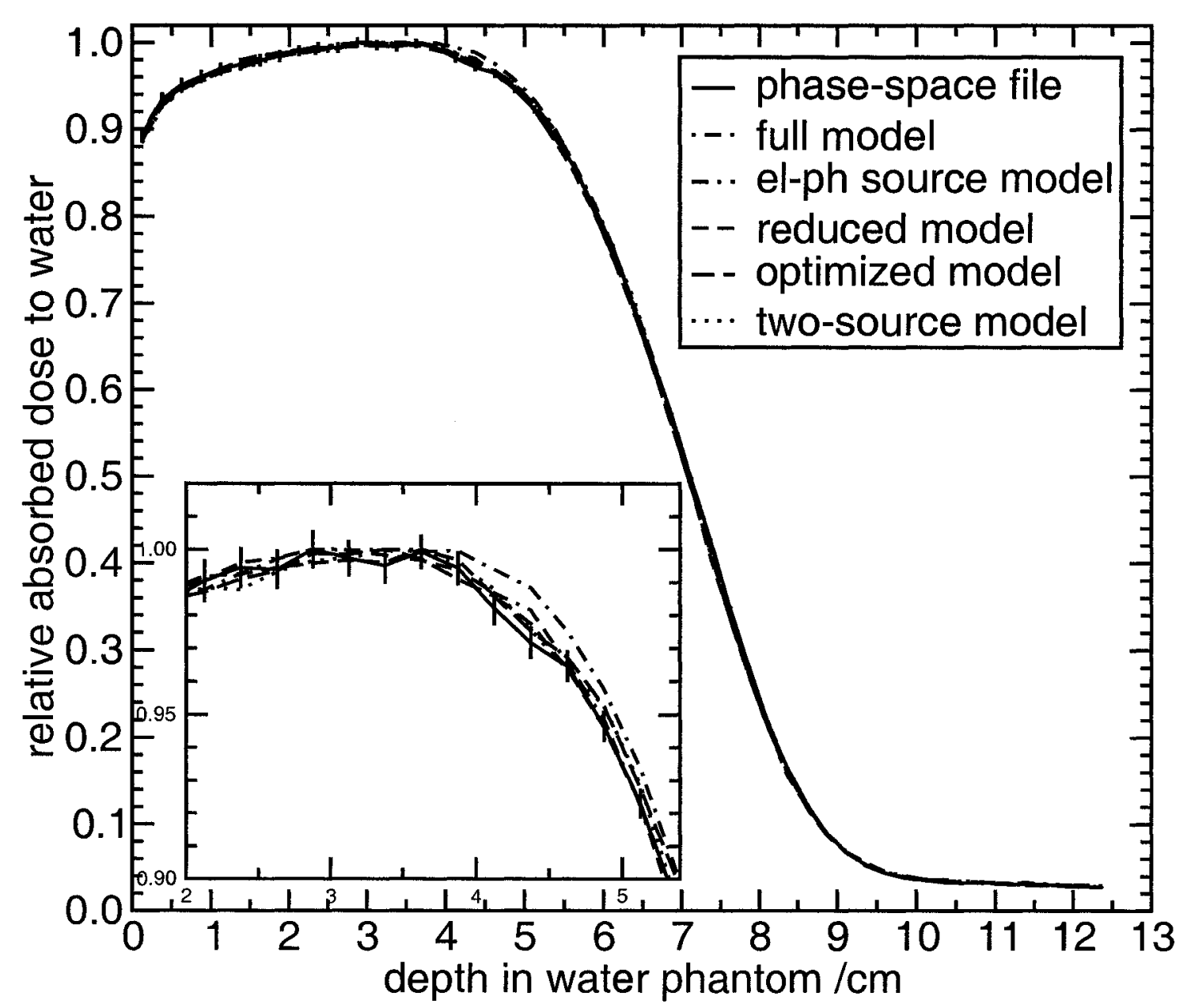

Figure 6.1: Comparison between central-axis depth-dose curves for $18 \mathrm{MeV}$ electron beam, $10 \times 10 \mathrm{~cm}^{2}$ field, calculated using original phase-space file and five multiplesource models. The same number of histories were used for all depth-dose calculations. 
ment between the models and original phase-space file is well within the statistics of $0.5 \%$ for the full sub-source models. The dose distribution calculated using the two-source model deviates less than $1 \%$ from that calculated using the original phasespace file. The central-axis depth-dose calculation from a one-electron-source model

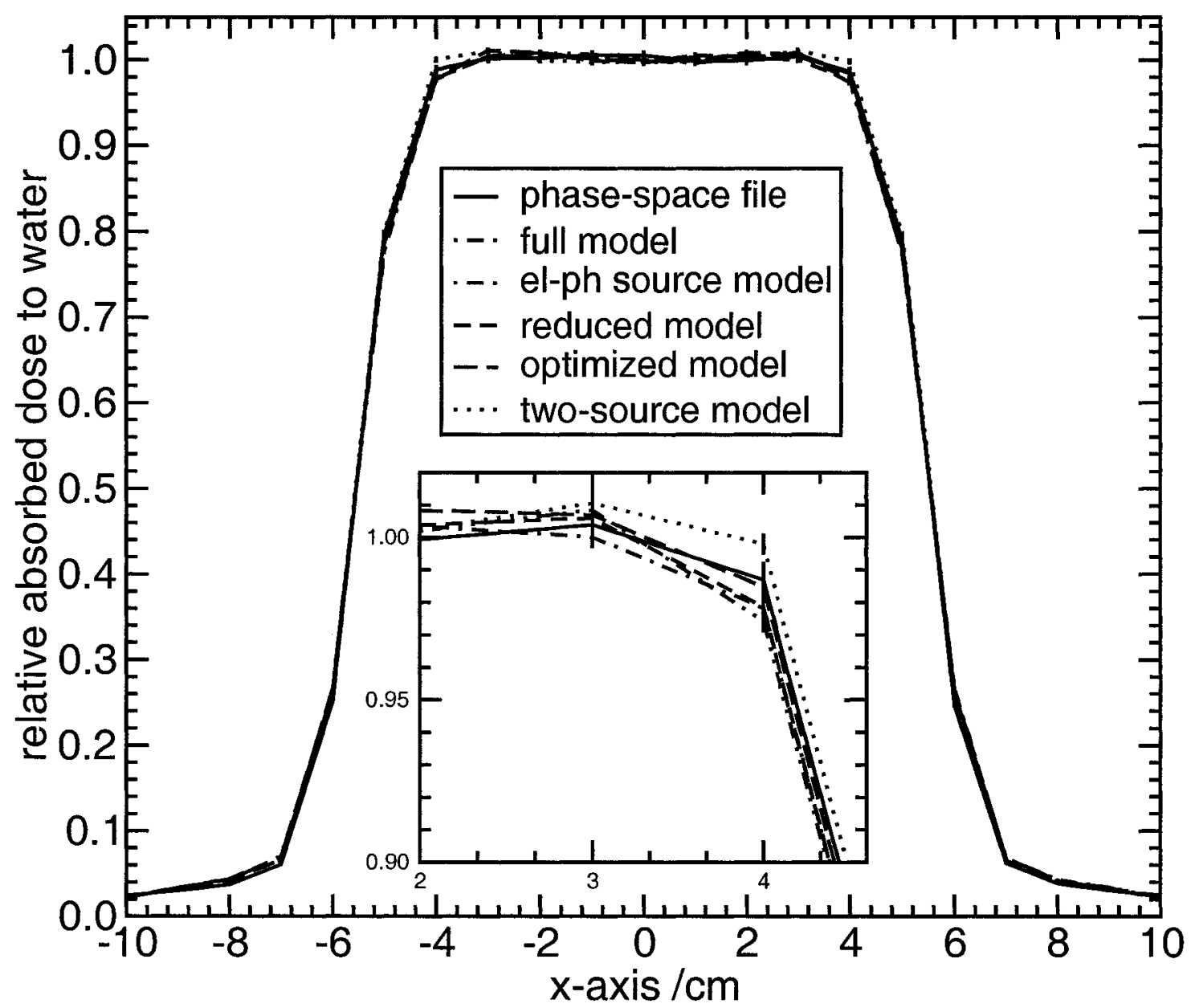

Figure 6.2: Comparison between dose-profiles curves for $18 \mathrm{MeV}$ electron beam, $10 \times 10 \mathrm{~cm}^{2}$ field, calculated using original phase-space file and five multiple-source models at $d_{\max }=3.20 \mathrm{~cm}$ depth

is shown in Figure 6.3. It is evident, by the slope of the curve and dose underestimate

\subsection{ELECTRON BEAM}


in the photon tail, that photons incident on the phantom are not accounted for in this model, and that the photon contribution to the absorbed dose is greater at deeper depths. Figure 6.4 presents the difference in central-axis depth-dose calculation

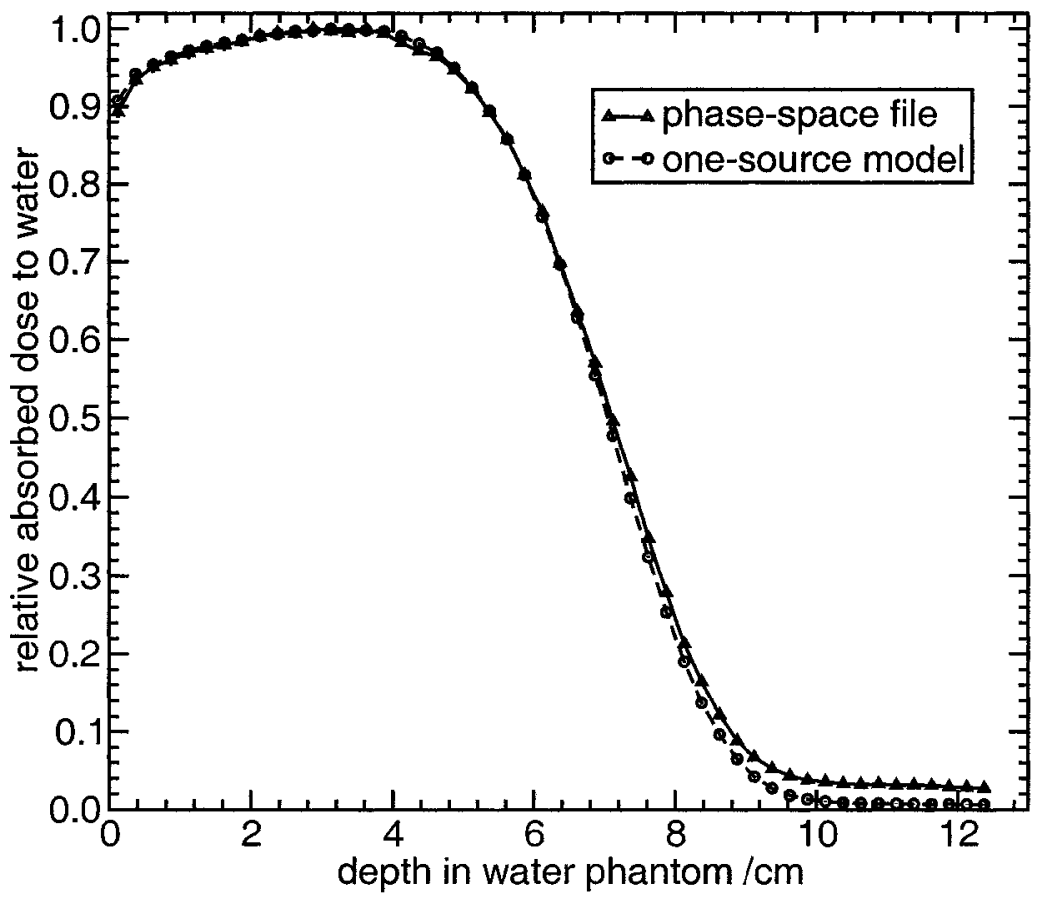

Figure 6.3: Comparison between central-axis depth-dose curves for $18 \mathrm{MeV}$ electron beam, $10 \times 10 \mathrm{~cm}^{2}$ field size, calculated using the original phase-space file and the onesource model for electrons. The slope of the curve and photon tail do not agree since no photon sources are present in this model.

between the new and old BEAMDP code for the full model for the $18 \mathrm{MeV}$ electron beam, $10 \times 10 \mathrm{~cm}^{2}$ field size using the same number of histories for all three calculations which give the similar results within statistics on both models. Significant differences between the new and old BEAMDP were not expected for electron beams since the mean energy is flat inside and outside of the treatment field (Figure 6.5).

\subsection{ELECTRON BEAM}




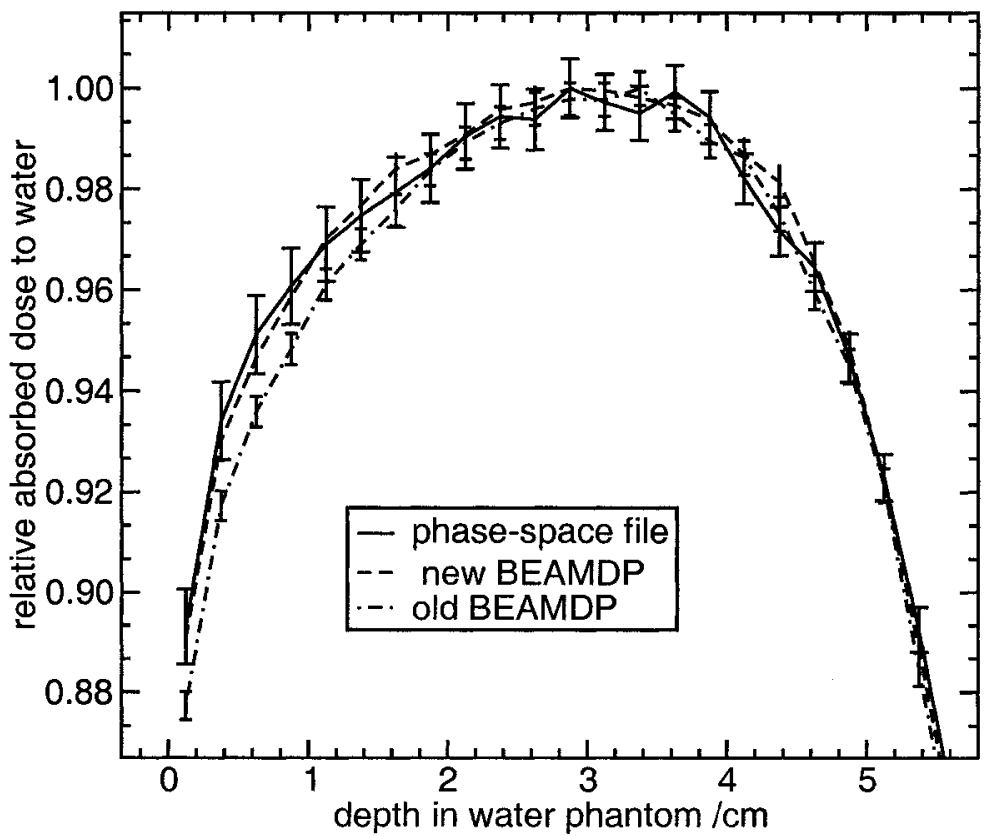

Figure 6.4: Comparison between central-axis depth-dose curves for $18 \mathrm{MeV}$ electron beam, $10 \times 10 \mathrm{~cm}^{2}$ field size, calculated using the original phase-space file and full multiple-source models for electrons developed with the new and old BEAMDP and associated MORTRAN replacement macros.

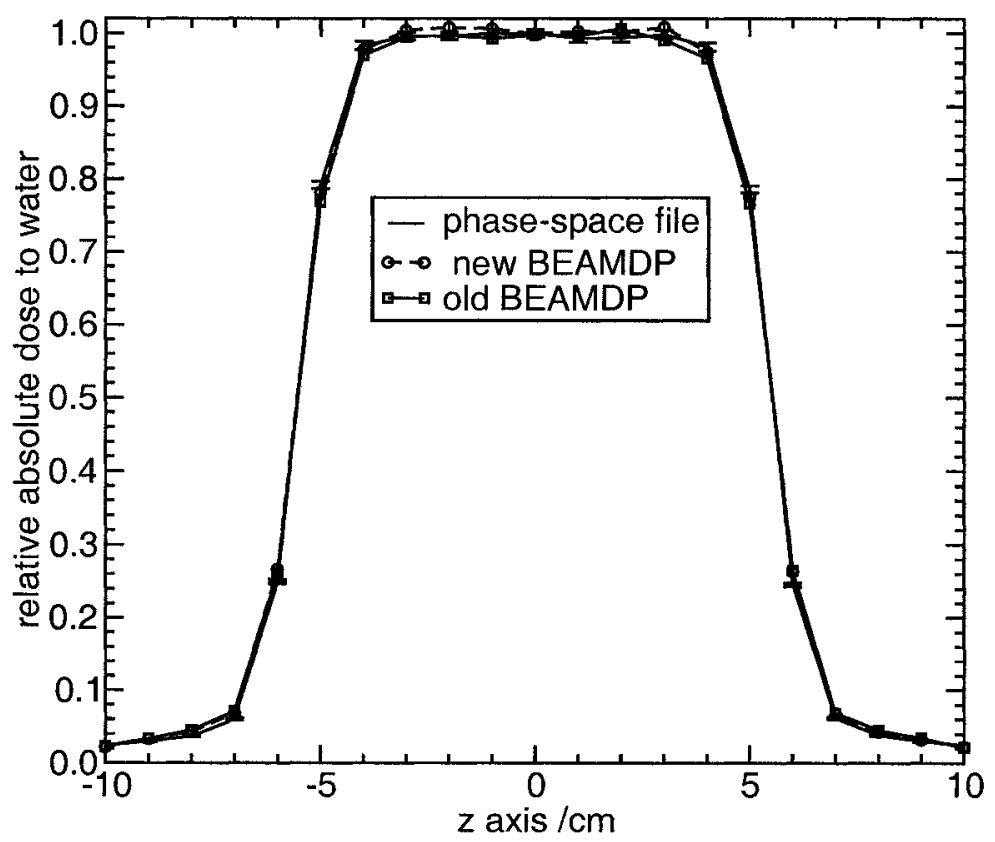

Figure 6.5: Comparison between dose-profiles curves for $18 \mathrm{MeV}$ electron beam, $10 \times 10 \mathrm{~cm}^{2}$ field, calculated using original phase-space file and old and new BEAMDP codes for the full multiple-source models at $d_{\max }=3.20 \mathrm{~cm}$ depth.

\subsection{ELECTRON BEAM}




\subsection{Photon beam}

\subsubsection{Models}

Components of the full multiple-source model for the $25 \mathrm{MV}$ photon beam, $10 \times 10 \mathrm{~cm}^{2}$ field size, are presented in Table 6.7. There are a total of 15 components, 9 for photons, 4 for electrons and 2 for positrons. There is only one point source, for photons, since electrons and positrons cannot get through the flattening filter from the target. The distance for the point source from the scoring plane is calculated by the pinhole method in the BEAMDP code (explained in Section 3.5). Other photon sources are from the primary collimator (LATCH bits 4), flattening filter (LATCH bits 5), monitor chamber (LATCH bit 7), backscatter plate (LATCH bit 8), mirror (LATCH bit 9), jaws (LATCH bits 13 and 14) and mylar screen (LATCH bit 15). Electron sources are from the flattening filter (LATCH bit 5), jaws (LATCH bits 13 and 14) and air (LATCH bits 23). As explained at the end of the previous chapter, electrons created in the air by any photons inherit LATCH bit 1 and has LATCH bit 23 set. A rectangular plane sub-source was used, assigned to the LATCH bit 1, positioned at the same distance as the mylar screen. The same applies for positrons. Positrons make up 20\% of charged particles and they can not be ignored since their contribution at the surface is not negligible. About $67 \%$ of positrons are created in the air and $33 \%$ in the flattening filter (Figure 2.1). Electrons make up about $80 \%$ of the charged particles in the beam. About $56 \%$ of electrons are created in the air with $20 \%$ coming directly from the jaws and $23 \%$ from the flattening filter.

Photon sub-sources from the monitor chamber, mirror, backscatter plate and mylar screen have very low intensities, and these sources can be eliminated. Components for this model are given in Table 6.8. Components for the further reduced model

\subsection{PHOTON BEAM}


Table 6.7: Components of the full multiple-source model for $25 \mathrm{MV}$ photon beam, $10 \times 10 \mathrm{~cm}^{2}$ field size. Ch is charge of the sub-source, L is LATCH bit, $\mathrm{d}$ is the distance from the sub-source to the scoring plane, 'PRI' is relative intensity per particle type. The model contains 15 sub-sources, 9 for photons, 4 for electrons and 2 for positrons.

\begin{tabular}{llllllll}
\hline \hline Source & Source type & Component & Ch & L & d/cm & RI/\% & PRI/\% \\
\hline 1 & Point source & Target & 0 & 1 & 100 & 93.00 & 94.15 \\
2 & Circ.plane & Primary collimator & 0 & 4 & 93 & 2.46 & 2.49 \\
3 & Circ.plane & Flattening filter & 0 & 5 & 85 & 1.95 & 1.98 \\
4 & Circ.plane & Flattening filter & -1 & 5 & 85 & 0.23 & 22.70 \\
5 & Circ.plane & Flattening filter & 1 & 5 & 85 & 0.07 & 32.52 \\
6 & Circ.plane & Ion chamber & 0 & 7 & 83 & 0.01 & 0.01 \\
7 & Circ.plane & Backscatter plate & 0 & 8 & 82 & 0.16 & 0.16 \\
8 & Rect.plane & Mirror & 0 & 9 & 77 & 0.00 & 0.00 \\
9 & Collimator & Jaws & -1 & 13 & 67 & 0.10 & 9.68 \\
10 & Collimator & Jaws & 0 & 13 & 67 & 0.62 & 0.63 \\
11 & Collimator & Jaws & -1 & 14 & 55 & 0.10 & 10.14 \\
12 & Collimator & Jaws & 0 & 14 & 55 & 0.56 & 0.57 \\
13 & Circ.plane & Mylar screen & 0 & 15 & 47 & 0.01 & 0.01 \\
14 & Rect.plane & Air & -1 & 1 & 47 & 0.58 & 57.48 \\
15 & Rect.plane & Air & 1 & 1 & 47 & 0.15 & 67.48 \\
\hline \hline
\end{tabular}


Table 6.8: Components of the reduced full multiple-source model (marked as 1) for $25 \mathrm{MV}$ photon beam, $10 \times 10 \mathrm{~cm}^{2}$ field size. The model contains 11 sub-sources, 5 for photons, 4 for electrons and 2 for positrons.

\begin{tabular}{llllllll}
\hline \hline Source & Source type & Component & Ch & L & d/cm & RI/\% & PRI/\% \\
\hline 1 & Point source & Target & 0 & 1 & 100 & 93.00 & 94.15 \\
2 & Circ.plane & Primary collimator & 0 & 4 & 93 & 2.46 & 2.49 \\
3 & Circ.plane & Flattening filter & 0 & 5 & 85 & 2.12 & 2.15 \\
4 & Circ.plane & Flattening filter & -1 & 5 & 85 & 0.23 & 22.70 \\
5 & Circ.plane & Flattening filter & 1 & 5 & 85 & 0.07 & 32.52 \\
6 & Collimator & Jaws & -1 & 13 & 67 & 0.10 & 9.68 \\
7 & Collimator & Jaws & 0 & 13 & 67 & 0.62 & 0.63 \\
8 & Collimator & Jaws & -1 & 14 & 55 & 0.10 & 10.14 \\
9 & Collimator & Jaws & 0 & 14 & 55 & 0.57 & 0.58 \\
10 & Rect.plane & Air & -1 & 1 & 47 & 0.58 & 57.48 \\
11 & Rect.plane & Air & 1 & 1 & 47 & 0.15 & 67.48 \\
\hline \hline
\end{tabular}


are given in Table 6.9. The weak jaws photon sub-sources have been eliminated. The

Table 6.9: Components of the further reduced multiple-source model for $25 \mathrm{MV}$ photon beam, $10 \times 10 \mathrm{~cm}^{2}$ field size. The model contains 9 sources, 3 for photons, 4 for electrons and 2 for positrons.

\begin{tabular}{lllccccc}
\hline \hline Source & Source type & Component & $\mathrm{Ch}$ & $\mathrm{L}$ & $\mathrm{d} / \mathrm{cm}$ & $\mathrm{RI} / \%$ & $\mathrm{PRI} / \%$ \\
\hline 1 & Point source & Target & 0 & 1 & 100 & 93.00 & 94.15 \\
2 & Circ.plane & Primary collimator & 0 & 4 & 93 & 2.46 & 2.49 \\
3 & Circ.plane & Flattening filter & 0 & 5 & 85 & 3.31 & 3.36 \\
4 & Circ.plane & Flattening filter & -1 & 5 & 85 & 0.23 & 22.70 \\
5 & Circ.plane & Flattening filter & 1 & 5 & 85 & 0.07 & 32.52 \\
6 & Collimator & Jaws & -1 & 13 & 67 & 0.10 & 9.68 \\
7 & Collimator & Jaws & -1 & 14 & 55 & 0.10 & 10.14 \\
8 & Rect.plane & Air & -1 & 1 & 47 & 0.58 & 57.48 \\
9 & Rect.plane & Air & 1 & 1 & 47 & 0.15 & 67.48 \\
\hline \hline
\end{tabular}

optimized, five-source model, which contains only the point source for photons, flattening filter and air sub-sources for electrons and positrons is presented in Table 6.10. A central-axis depth-dose comparison for this model against the original phase-space file is shown in Figure 6.8. The most simplified model is the model which contains only three sources, one point source for photons and two planar electron and positron sources (Table 6.11). 
Table 6.10: Components of the optimized multiple-source model for $25 \mathrm{MV}$ photon beam, $10 \times 10 \mathrm{~cm}^{2}$ field size. The model contains 5 sub-sources, 1 for photons, 2 for electrons and 2 for positrons.

\begin{tabular}{llllllll}
\hline \hline Source & Source type & Component & $\mathrm{Ch}$ & $\mathrm{L}$ & $\mathrm{d} / \mathrm{cm}$ & $\mathrm{RI} / \%$ & PRI/\% \\
\hline 1 & Point source & Target & 0 & 1 & 100 & 98.77 & 100.00 \\
2 & Circ.plane & Flattening filter & -1 & 5 & 85 & 0.43 & 42.52 \\
3 & Circ.plane & Flattening filter & 1 & 5 & 85 & 0.07 & 32.52 \\
4 & Rect.plane & Air & -1 & 1 & 47 & 0.58 & 57.48 \\
5 & Rect.plane & Air & 1 & 1 & 47 & 0.15 & 67.48 \\
\hline \hline
\end{tabular}

Table 6.11: Components of the three-source model for $25 \mathrm{MV}$ photon beam, $10 \times 10 \mathrm{~cm}^{2}$ field size. The model contains 1 point sub-source for photons and two planar sub-sources for electrons and positrons.

\begin{tabular}{lllccccc}
\hline \hline Source & Source type & Component & $\mathrm{Ch}$ & $\mathrm{L}$ & $\mathrm{d} / \mathrm{cm}$ & $\mathrm{RI} / \%$ & $\mathrm{PRI} / \%$ \\
\hline 1 & Point source & Target & 0 & 1 & 100 & 98.77 & 100.00 \\
2 & Rect.plane & Air & -1 & 1 & 47 & 1.00 & 100.00 \\
3 & Rect.plane & Air & 1 & 1 & 47 & 0.23 & 100.00 \\
\hline \hline
\end{tabular}




\subsubsection{Depth-dose and dose-profile comparisons}

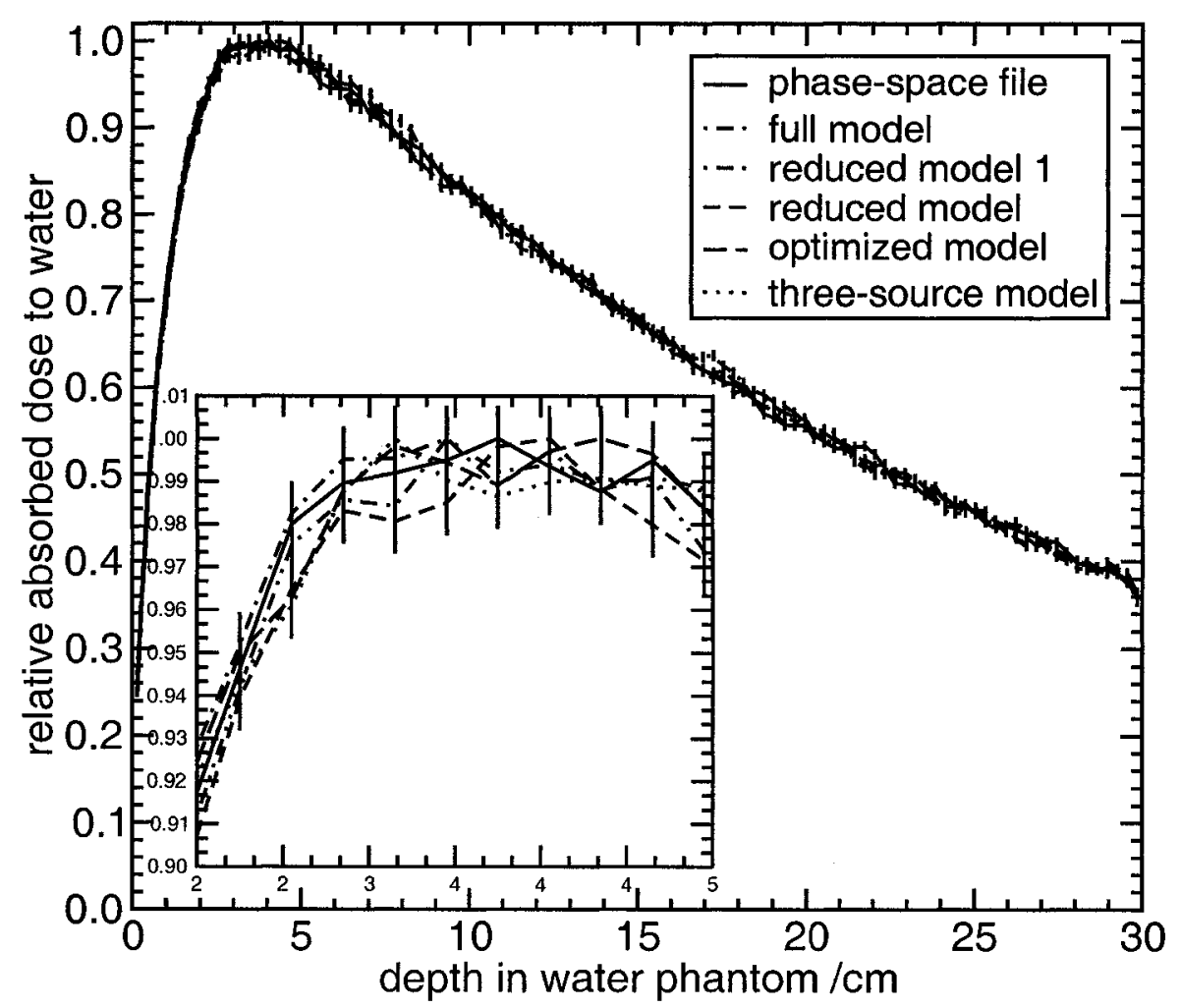

Figure 6.6: Comparison between central-axis depth-dose curves for $25 \mathrm{MV}$ photon beam, $10 \times 10 \mathrm{~cm}^{2}$ field, calculated using original phase-space file and five multiplesource models

Central-axis depth-dose comparison for the $25 \mathrm{MV}$ photon beam, $10 \times 10 \mathrm{~cm}^{2}$ field, calculated using the original phase-space file and five multiple-source models shows good agreement for all models as shown in Figure 6.6. From Figure 6.7 it is observed that agreement between dose-profiles from the models and phase-space file is well within statistics for all models. 


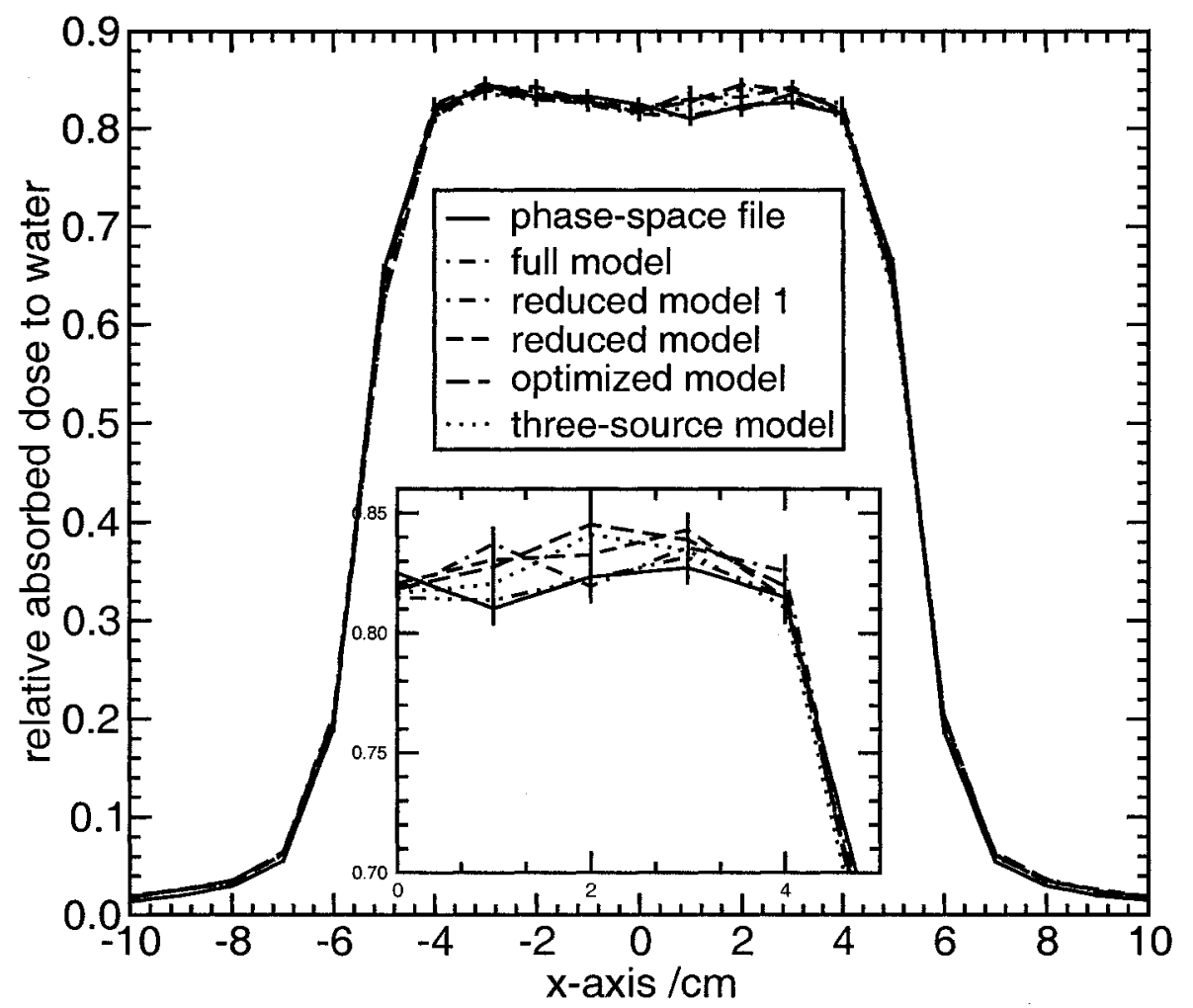

Figure 6.7: Comparison between dose-profiles curves for the $25 \mathrm{MV}$ photon beam, $10 \times 10 \mathrm{~cm}^{2}$ field size, calculated using original phase-space file and five multiple-source models at $d=10 \mathrm{~cm}$ depth in the water phantom. 


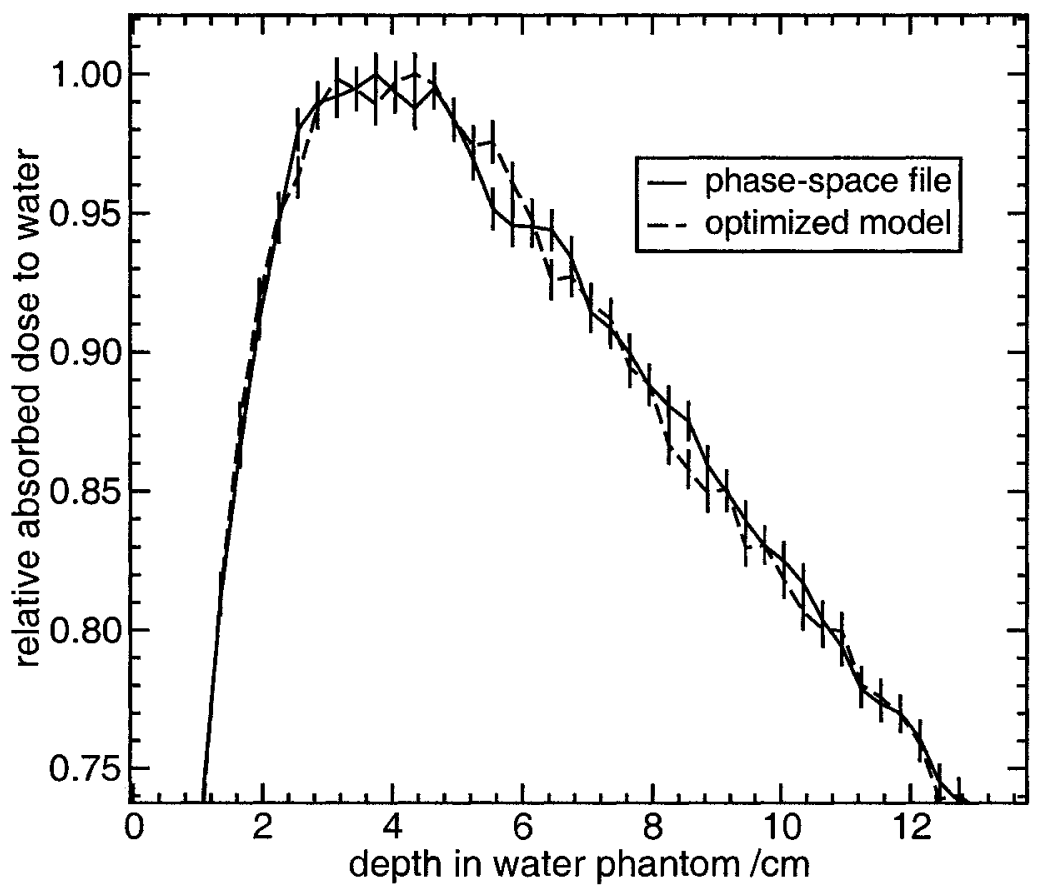

Figure 6.8: Comparison between central-axis depth-dose curves for the $25 \mathrm{MV}$ photon beam, $10 \times 10 \mathrm{~cm}^{2}$ field, calculated using original phase-space file and optimized multiple-source models.

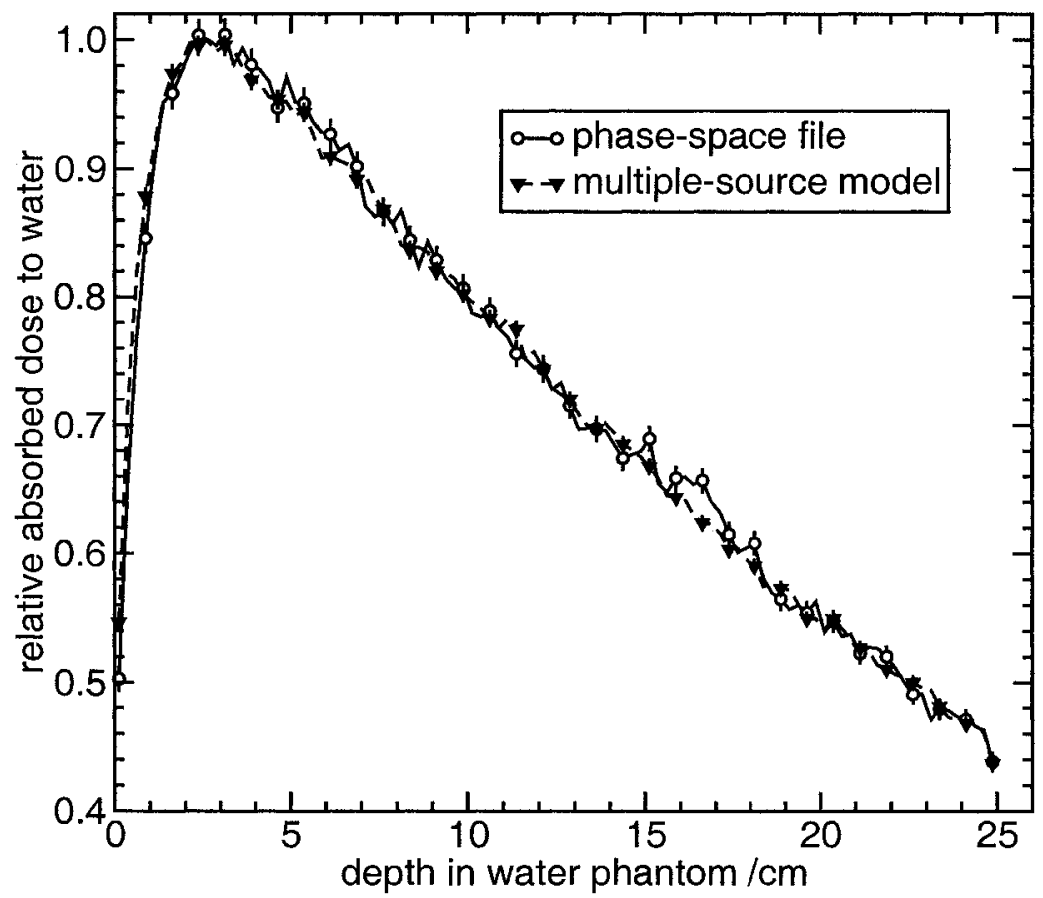

Figure 6.9: Relative absorbed dose to water for the $25 \mathrm{MV}$ broad photon beam calculated using phase-space file and full multiple-source model 
The effect on depth-dose curves of ignoring all charged particles in the 25 MV photon beam, $10 \times 10 \mathrm{~cm}^{2}$ field size, is evident from Figure 6.10, the electron contribution to the absorbed dose is at shallower depths.

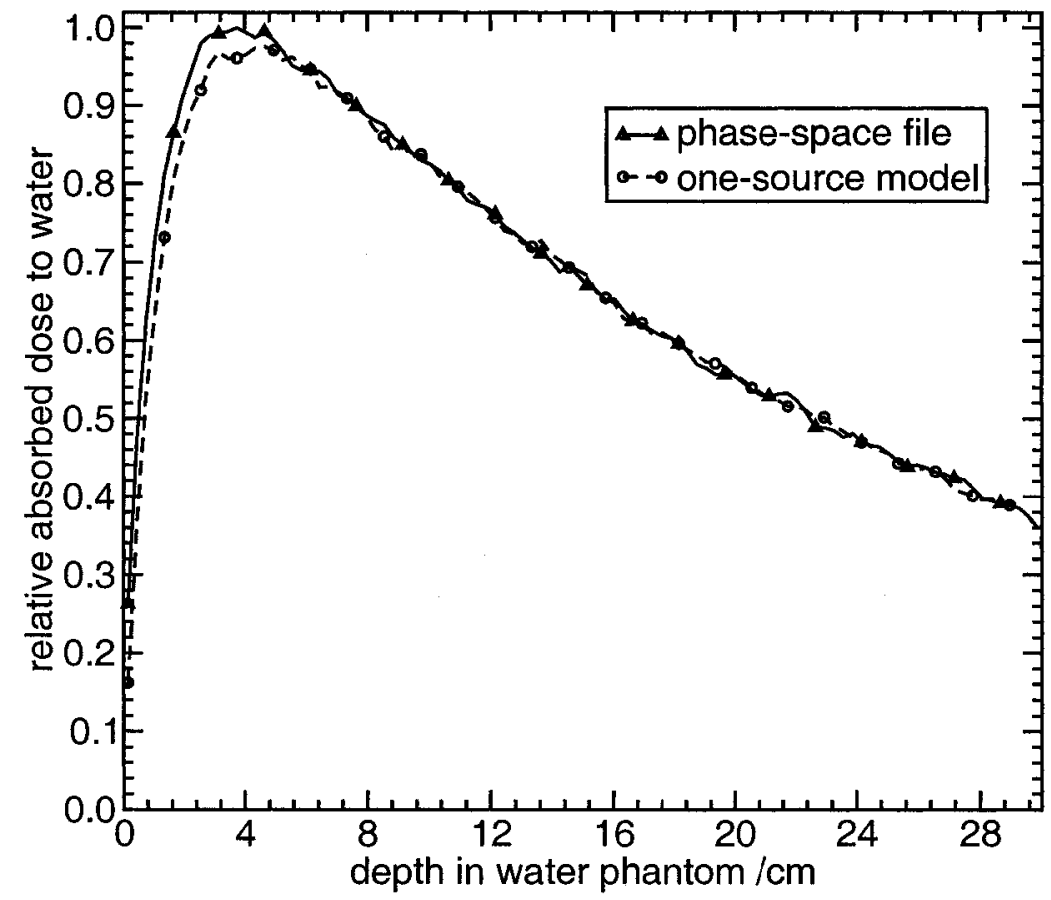

Figure 6.10: Comparison between central-axis depth-dose curves for the $25 \mathrm{MV}$ photon beam, $10 \times 10 \mathrm{~cm}^{2}$ field size, calculated using original phase-space file and onesource model for photons. One-source model normalized to phase-space calculation at $10 \mathrm{~cm}$.

Figure 6.11 shows the effect of multiple-energy regions on dose-profiles for a photon beam. Variation of the mean energy inside the treatment field is significant and the old BEAMDP approximation of a single spectrum inside the field does not give good results for the dose profile of any energy photon beam. The new BEAMDP code with multiple-region energy spectra removes this shortcoming.

\subsection{PHOTON BEAM}




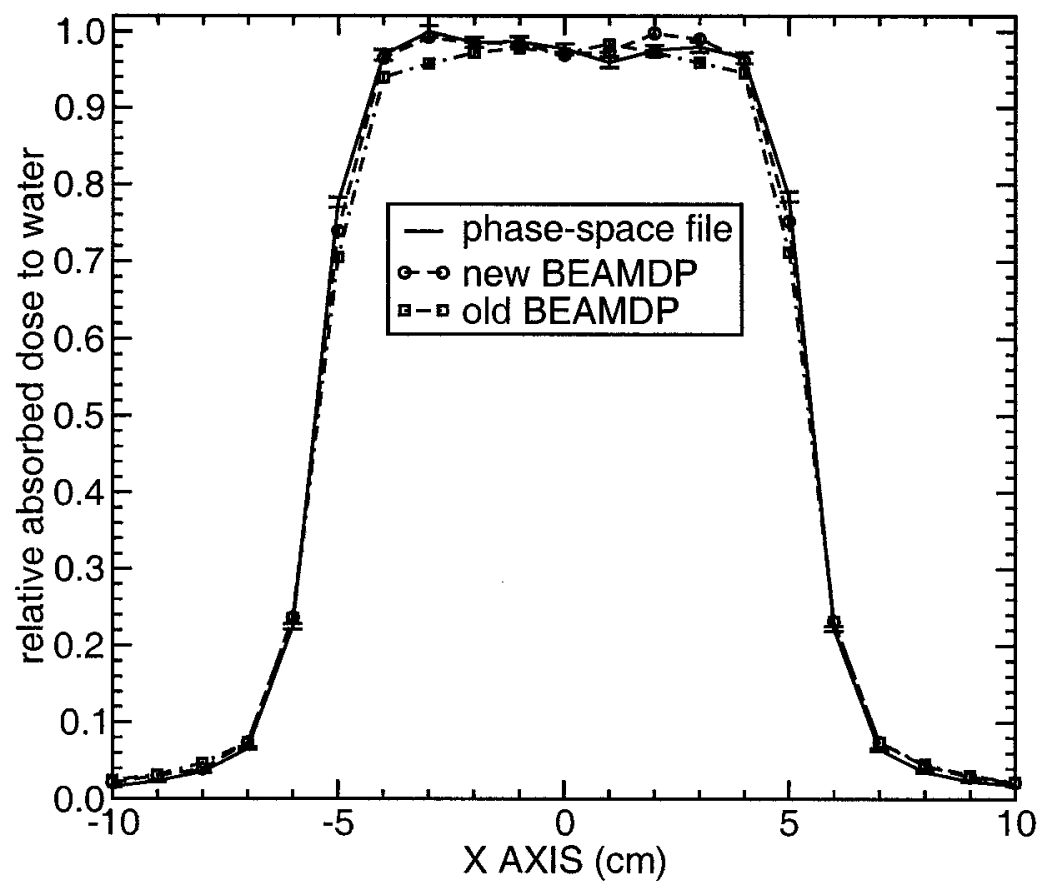

Figure 6.11: Comparison between dose-profiles curves for the 25 MV photon beam, $10 \times 10 \mathrm{~cm}^{2}$ field size, calculated using original phase-space file and two-source models at $d=10 \mathrm{~cm}$ depth in the water phantom. 


\section{Chapter 7}

\section{Conclusion}

The aim of this project was to develop a multiple-source model for the Elekta SL25 linear accelerator so that the model can be used for dosimetry research purposes on the linac located at NRC, and to investigate the accuracy in dose calculation using multiple-source models.

The BEAMDP code and the MORTRAN beam model replacement macros have been modified for this project to improve the accuracy of multiple-source models. The original BEAMDP code was developed in 1997 at NRC. The new functionality that has been added to the code needed thorough testing.

For both, electron and photons beams, five different models have been developed with different numbers of sub-sources, ranging from 21 to only 2 . It was found that the calculated dose distributions in a water phantom were in good agreement with those calculated using the original phase-space file for all models within about $0.5 \%$ to $1 \%$ accuracy.

Multiple-source models give good results, including two-source models which 
include only two point sources, and no geometry details from the linear accelerator. This model can be easily created for any beam and still be an accurate alternative for dose calculations, negligible in size compared with size of phase-space file. The chart on Figure 7.1 compares the size of phase-space file (390 MB) versus size of multiplesource model file (0.2 MB for two sub-sources) for the same energy of $18 \mathrm{MeV}$ and $10 \times 10 \mathrm{~cm}^{2}$ field size.

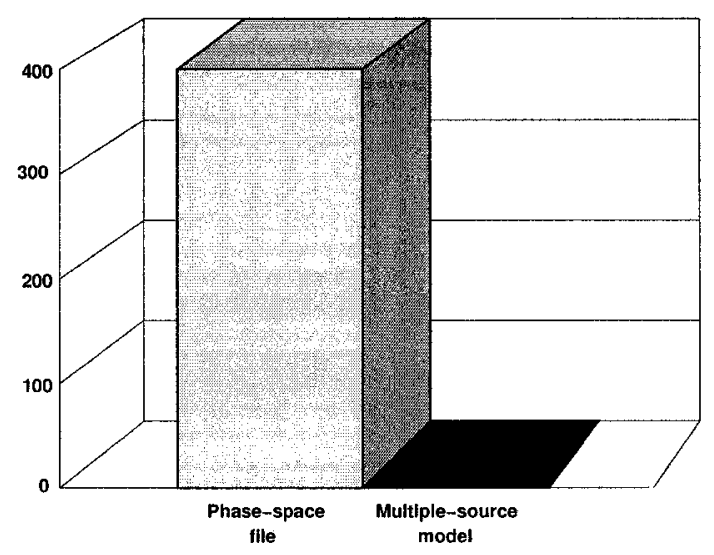

Figure 7.1: Disk space requirements (in MB) for phase-space file versus multiplesource model file for the same energy of $18 \mathrm{MeV}, 10 \times 10 \mathrm{~cm}^{2}$ field size and 20 million histories 


\section{Bibliography}

[1] C.-M. Ma and D. W. O. Rogers. BEAMDP Users Manual. NRC Report PIRS 509c, 1995.

[2] C.-M. Ma, B. A. Faddegon, D. W. O. Rogers, and T. R. Mackie. Accurate characterization of Monte Carlo calculated electron beams for radiotherapy. Med. Phys., 24:401 - 416, 1997.

[3] S. B. Jiang, A. Kapur, and C-M. Ma. Electron beam modeling and commissioning for Monte Carlo treatment planning. Med. Phys., 27:180 - 191, 2000.

[4] A. Chaves, M. C. Lopes, C. C. Alves, C. Oliveira, L. Peralta, P. Rodrigues, and A. Trindade. Basic dosimetry of radiosurgery narrow beams using Monte Carlo simulations: A detailed study of depth of maximum dose. Med. Phys., 30:2904-2911, 2003.

[5] I. J. Chetty et al. Issues associated with clinical implementation of Monte Carlobased treatment planning: Report of the AAPM Task Group No. 105 . Med. Phys., 34:(submitted), 2006.

[6] I. Kawrakow and D. W. O. Rogers. The EGSnrc Code System: Monte Carlo simulation of electron and photon transport. Technical Report PIRS-701, National 
Research Council of Canada, Ottawa, Canada, 2000.

[7] D. W. O. Rogers, B. A. Faddegon, G. X. Ding, C.-M. Ma, J. Wei, and T. R. Mackie. BEAM: A Monte Carlo code to simulate radiotherapy treatment units. Med. Phys., 22:503 - 524, 1995.

[8] R. L. Ford and W. R. Nelson. The EGS code system - Version 3. Report SLAC-210, Stanford Linear Accelerator Center, Stanford, California, 1978.

[9] W. R. Nelson, H. Hirayama, and D. W. O. Rogers. The EGS4 Code System. Report SLAC-265, Stanford Linear Accelerator Center, Stanford, California, 1985.

[10] I. Kawrakow and D. W. O. Rogers. The EGSnrc Code System: Monte Carlo simulation of electron and photon transport. Technical Report PIRS-701, $\mathrm{Na}$ tional Research Council of Canada, Ottawa, Canada, page (2000). , see http://www.irs.inms.nrc.ca/inms/irs/EGSnrc/EGSnrc.html.

[11] M. J. Berger. Monte Carlo Calculation of the penetration and diffusion of fast charged particles. In B. Alder, S. Fernbach, and M. Rotenberg, editors, Methods in Comput. Phys., volume 1, pages 135 - 215. Academic, New York, 1963.

[12] S. M. Seltzer. Electron-photon Monte Carlo calculations: the ETRAN code. Int'l J of Appl. Radiation and Isotopes, ,42:917 - 941, 1991.

[13] J. Halbleib. Structure and Operation of the ITS code system. In T. M. Jenkins, W. R. Nelson, A. Rindi, A. E. Nahum, and D. W. O. Rogers, editors, Monte Carlo Transport of Electrons and Photons, pages 249 - 262. Plenum Press, New York, 1988. 
[14] F. B. Brown (Editor). MCNP-A general Monte Carlo N-particle transport code Version 5. Report LA-UR-03-1987, Los Alamos National Laboratory, Los Alamos, NM, 2003.

[15] F. Salvat, J. M. Fernandez-Varea, and J. Sempau. PENELOPE - A Code System for Monte Carlo Simulation of Electron and Photon Transport. Technical report, OECD Nuclear Energy Agency, Issy-les-Moulineaux, France, 2003.

[16] J. Sempau, E. Acosta, J. Baro, J. M. Fernandez-Varea, and F. Salvat. An algorithm for Monte Carlo simulation of coupled electron-photon transport. Nucl. Inst. Meth. B, 132, 1997.

[17] M. Fippel. Fast Monte Carlo dose calculation for photon beams based on the VMC electron algorithm. Med. Phys., 26:1466 - 1475, 1999.

[18] I. Kawrakow and M. Fippel. Investigation of variance reduction techniques for Monte Carlo photon dose calculation using XVMC. Phys. Med. Biol., 45:2163$2184,2000$.

[19] J. Sempau, S. J. Wilderman, and A. F. Bielajew. DPM, a fast, accurate Monte Carlo code optimized for photon and electron radiotherapy treatment planning dose calculations. Phys. Med. Biol., 45:2263-2291, 2000.

[20] I. Kawrakow. Accurate condensed history Monte Carlo simulation of electron transport. II. Application to ion chamber response simulations. Med. Phys., $27: 499-513,2000$.

[21] J. P. Seuntjens, I. Kawrakow, J. Borg, F. Hobeila, and D. W. O. Rogers. Calculated and measured air-kerma response of ionization chambers in low and medium energy photon beams. In J. P. Seuntjens and P. Mobit, editors, Recent 
developments in accurate radiation dosimetry, Proc. of an Int'l Workshop, pages 69 - 84. Medical Physics Publishing, Madison WI, 2002.

[22] I. Kawrakow. Accurate condensed history Monte Carlo simulation of electron transport. I. EGSnrc, the new EGS4 version. Med. Phys., 27:485 - 498, 2000.

[23] I. Kawrakow, E. Mainegra-Hing, and D. W. O. Rogers. EGSnrcMP: the multiplatform environment for EGSnrc. Technical Report PIRS-877, National Research Council of Canada, Ottawa, Canada, 2003.

[24] E. Storm and H. I. Israel. Photon cross sections from $1 \mathrm{keV}$ to $100 \mathrm{MeV}$ for elements $Z=1$ to $Z=100$. Atomic Data and Nuclear Data Tables, 7:565 - 681, 1970.

[25] J. W. Motz, H. A. Olsen, and H. W. Koch. Pair production by photons. Rev. Mod. Phys., 41:581 - 639, 1969.

[26] M. J. Berger and R. Wang. Multiple-scattering angular deflections and energyloss straggling. In T. M. Jenkins, W. R. Nelson, A. Rindi, A. E. Nahum, and D. W. O. Rogers, editors, Monte Carlo Transport of Electrons and Photons, pages 21 - 56. Plenum Press, New York, 1988.

[27] I. Kawrakow and A. F. Bielajew. On the condensed history technique for electron transport. Nuclear Instruments and Methods, 142B:253-280, 1998.

[28] G. Marsaglia, A. Zaman, and W. W. Tsang. Toward a universal random number generator. Statistics and Probability Letters, 8:35 - 39, 1990.

[29] G. Marsaglia and A. Zaman. A new class of random number generators. Annals of Applied Probability, 1:462 - 480, 1991.

BIBLIOGRAPHY 
[30] M. Lüscher. A portable high-quality random number generator for lattice field theory simulations. Computer Phys. Commun., 79:100 - 110, 1994.

[31] F. James. RANLUX: A Fortran implementation of the high-quality pseudorandom number generator of Lüscher. Computer Phys. Commun., 79:111 - 114, 1994.

[32] D. W. O. Rogers, C.-M. Ma, G. X. Ding, B. Walters, D. Sheikh-Bagheri, and G. G. Zhang. BEAMnrc Users Manual. NRC Report PIRS 509(a)revF, 2001.

[33] R. Brun, O. Couet, C. Vandoni, and P. Zanarini. PAW User Guide. CERN Computer Center, Geneva, Switzerland, 1992.

[34] B. R. B. Walters, I. Kawrakow, and D. W. O. Rogers. History by history statistical estimators in the BEAM code system. Med. Phys., 29:2745 - 2752, 2002.

[35] J. Sempau, A. Sánchez-Reyes, F. Salvat, H. Oulad ben Tahar, S. B. Jiang, and J. M. Fernández-Varea. Monte Carlo simulation of electron beams from an accelerator head using PENELOPE. Phys. Med. Biol., 46:1163 - 1186, 2001.

[36] C.-M. Ma, D. W. O. Rogers, and B. Walters. DOSXYZnrc Users Manual. NRC Report PIRS 509b(revF), 2001.

[37] H. C. E. McGowan, B. A. Faddegon, and C-M Ma. STATDOSE for 3D dose distributions. NRC Report PIRS 509f, 1995.

[38] A. Janssens, E. W. Korevaar, L. J. van Battum, P. R.Storchi, and H. Huizenga. A model to determine the initial phase space of a clinical electron beam from measured beam data. Phys. Med. Biol., 46:269 - 286, 2001. 
[39] P. L. Roberson S. Yokoyama, D. L. Litzenberg, J. M. Moran, and B. A. Fraass. Surface buildup dose dependence of photon field delivery technique for IMRT . $J$ of App Clin Med Phys, 5:71 - 81, 2004.

[40] T. Kron, A. Elliot, T. Wong, G. Showell, B. Clubb, and P. Metcalfe. X-ray surface dose measurements using TLD extrapolation . Med. Phys., 20:703 - 711, 1993.

[41] D. T. Burns, G. X. Ding, and D. W. O. Rogers. $R_{50}$ as a beam quality specifier for selecting stopping-power ratios and reference depths for electron dosimetry. Med. Phys., 23:383 - 388, 1996.

[42] D. Sheikh-Bagheri and D. W. O. Rogers. Sensitivity of megavoltage photon beam Monte Carlo simulations to electron beam parameters. Med. Phys., 29:379 $-390,2002$.

[43] B. A. Faddegon, J. Balogh, R. Mackenzie, and D. Scora. Clinical considerations of Monte Carlo for electron radiotherapy treatment planning. Radiation Physics and Chemistry, 53:217 - 227, 1998.

[44] E. Tonkopi, M. McEwen, B.R.B. Walters, and I. Kawrakow. Influence of ion chamber response on in-air profile measurements in megavoltage photon beams. Med. Phys., 32:2918-2927, 2005.

[45] B. A. Faddegon and I. Blevis. Electron spectra derived from depth dose distributions. Med. Phys., 27:514 - 526, 2000.

[46] P Björk, T Knöös, and P Nilsson. Influence of initial electron beam characteristics on Monte Carlo calculated absorbed dose distributions for linear accelerator beams. Phys. Med. Biol., 47:4019 - 4041, 2002.

BIBLIOGRAPHY 
[47] J. O. Deasy, P. R. Almond, and M. T. McEllistrem. The spectra dependence of electron central-axis depth-dose curves. Med. Phys., 21:1369 - 1376, 1994.

[48] P. Björk, P Nilsson, and T Knöös. Dosimetry characteristics of degraded electron beams investigated by Monte Carlo calculations in a setup for intraoperative radiation therapy. Phys. Med. Biol., 47:239 - 256, 2002.

[49] D. W. O. Rogers, C.-M. Ma, G. X. Ding, B. Walters, D. Sheikh-Bagheri, and G. G. Zhang. BEAM98 Users Manual. NRC Report PIRS 509(a)revC, 1998.

[50] D. Sheikh-Bagheri. Monte Carlo study of photon beams from medical linear accelerators; optimization, benchmark and spectra. PhD. thesis, Carleton University, Ottawa, 1999.

[51] B. R. B. Walters and D. W. O. Rogers. DOSXYZnrc Users Manual. NRC Report PIRS 794, 2002. 